\title{
Molecular Dynamics: a study on slip, drops, and graphene
}

by

Keoni Kealoha Mahelona

\author{
A thesis \\ submitted to the Victoria University of Wellington \\ in fulfillment of the \\ requirements for the degree of \\ Master of Science \\ in Physics.
}

Victoria University of Wellington

2011 



\section{Abstract}

Molecular dynamics (MD) is a computational tool used to study physical systems by modeling the atomic-scale interactions between atoms. MD can accurately predict the properties of materials where models are well developed. For new materials, models may be in their early stages and may lack the ability to produce accurate results; however, MD can still provide insight into the physical properties of these new materials. This thesis will use MD to study two different systems. First, the Lennard-Jones (L-J) liquid is used to study how the intrinsic slip lengths of atomic sized surfaces add to produce an effective slip of a larger surface made up of these atomic constituents. The results show that the effective slip of a surface is dominated by its smallest slip, and these results show good agreement with a theory that predicts effective slip given the intrinsic slip and roughness of a surface. The L-J model is also used to investigate the rolling and sliding motion of viscous drops on super-hydrophobic surfaces. The effects of drop size, slip length, and gravity on drop velocities are investigated, and a model that predicts drop speed given the characteristics of a drop and a surface is proposed. The model shows good agreement with simulation results, especially for certain regimes. Second, graphene is studied with MD using various atomistic models. The energies of layers of graphene are reproduced using an Adaptive Intermolecular Reactive Empirical Bond Order (AIREBO) potential, and the energies required to exfoliate graphene from crystal graphite and nickel nano-particles are calculated. The calculations from MD show good agreement with literature and experiment, and these results demonstrate how simple models in MD can produce useful results to aid research and experiment. Finally, the formation of nano-bubbles in graphene grown on platinum is studied using the AIREBO and L-J potentials. The basic formation of graphene nano-bubbles is demonstrated by compressing the edges of graphene flakes. The simulations highlight the importance of proper boundary conditions, such as atom pinning, in order to produce tall, smooth nano-bubbles. The results also suggest that accurate models will be required to effectively demonstrate bubble formation. 



\title{
Acknowledgements
}

\author{
Many thanks to:
}

- Fulbright for giving me the opportunity to study physics in Aotearoa;

- Fulbright New Zealand for supporting me during my time at university;

- Victoria University Scholarships Office for their financial support;

- the Hendy and Gaston research group for productive meetings, lengthly discussions, and delicious baked goods;

- Dr. Dmitri Schebarchov for code and thoughtful discussions;

- my advisor, Dr. Shaun Hendy, for all the advice and support provided during my research and for making my work enjoyable both inside and outside of "the lab;"

- ōku tūpuna, ōku whānau, me ōku hoa mō te aroha me te manaakitanga;

- a me ku'u ipo nō kōna aloha.

Mahalo nui loa kākou. 



\section{Contents}

Abstract

$\begin{array}{ll}\text { Acknowledgements } & \text { iii }\end{array}$

1 Introduction 1

2 Molecular Dynamics $\quad 5$

2.1 How MD Works . . . . . . . . . . . . . . . 5

2.2 Software . . . . . . . . . . . . . . . . . . 7

2.3 Important Considerations in $\mathrm{MD} \ldots \ldots \ldots . \ldots . \ldots 7$

3 Modeling Liquids $\quad 9$

3.1 The Lennard-Jones Liquid . . . . . . . . . . . . . . . . . . 9

3.2 Liquid Properties . . . . . . . . . . . . . . . . . . . . . 10

3.2.1 Separating Liquid from Vapor . . . . . . . . . . . . . 11

3.2 .2 Density . . . . . . . . . . . . . . . . . 13

3.2 .3 Surface Tension . . . . . . . . . . . . . . . . . 14

3.2 .4 Viscosity . . . . . . . . . . . . . . . . 17

3.3 Estimating Slip of Mixed Surfaces . . . . . . . . . . . . . . . 19

3.3.1 Theory . . . . . . . . . . . . . . . . 19

3.3 .2 Simulation . . . . . . . . . . . . . . . . . . . 21

3.3.3 Results . . . . . . . . . . . . . . . . 24

3.3 .4 Discussion . . . . . . . . . . . . . . 26

3.3.5 Conclusion . . . . . . . . . . . . . . . . . 28

3.4 Drop Dynamics on Slippery Surfaces . . . . . . . . . . . . . . . 29

3.4 Theory ..................... 30

3.4 .2 Simulation . . . . . . . . . . . . . 36

3.4 .3 Results and Analysis . . . . . . . . . . . . . . . . 37

3.4.4 Conclusion . . . . . . . . . . . . . . . . . 45 
4 Graphene $\quad 47$

4.1 Graphene in MD . . . . . . . . . . . . . . . . . . 47

4.2 Graphene Energies . . . . . . . . . . . . . . . . . . 49

4.2 .1 Motivation . . . . . . . . . . . . . . . 49

4.2 .2 Simulation and Results . . . . . . . . . . . . . 49

4.2 .3 Discussion and Conclusion . . . . . . . . . . . . . . 52

4.3 Graphene Nano-Bubbles . . . . . . . . . . . . . . . . . . 55

4.3.1 Motivation ................... 55

4.3 .2 Cause for Bubbles . . . . . . . . . . . . . . 56

4.3 .3 Simulation . . . . . . . . . . . . . . . 56

4.3.4 Results . . . . . . . . . . . . . . . . . 57

4.3.5 Discussion and Conclusion . . . . . . . . . . 61

5 Conclusion $\quad 65$

5.1 Liquids . . . . . . . . . . . . . . . . . . 65

5.1 .1 Effective Slip . . . . . . . . . . . . . . 66

5.1 .2 Rolling, Slipping Drops . . . . . . . . . . . . . 67

5.2 Graphene ......................... . . 68

\section{Appendices}

$\begin{array}{ll}\text { A Liquid Calculations } & 71\end{array}$

$\begin{array}{ll}\text { B Slip Length Simulations } & 73\end{array}$

$\begin{array}{ll}\text { C Drop Simulations } & 77\end{array}$

C.1 Measuring Contact Size . . . . . . . . . . . . . 77

C.2 Results ........................ 77

$\begin{array}{ll}\text { Bibliography } & 94\end{array}$ 


\section{Chapter 1}

\section{Introduction}

There may well be an infinite number of questions to ask of the physical world, but at any point in time only a finite number of those questions can be answered; however, as new technologies are developed, that finite number continues to growperhaps exponentially. The computer allows scientists and engineers to solve problems that cannot be solved without a computer (numerical solutions where solutions don't exist), that are beyond the scope of humankind (modeling the formation of solar systems), or that would be too risky to implement in real life (studying how wind currents carry nuclear fallout). As computers become more powerful, researchers not only answer more questions, but also they ask increasingly complex questions and questions that would previously take too long to answer.

Micro-scale and nano-scale systems are one particular area in which computer simulations aid researchers. Studying these smalls systems via experiment may require costly labs and equipment, or sometimes certain questions cannot be answered with today's technologies. Molecular dynamics (MD) is one of the many tools developed in order to probe the physical properties of these systems. MD can either mimic systems like fluids through simple models, or MD can accurately calculate properties of real systems. In this thesis, MD is used to explore the properties of two very different systems, liquids and graphene, showing the versatility of MD.

The flow of liquids along a surface is hindered by the atomistic interactions between a liquid and a solid as well as the roughness of the surface. Typically, surfaces are considered to completely stop fluid motion at the liquid-surface interface. This assumption is very accurate for most materials, and even with zero speed at the liquid-solid interface, we still have water flowing through our pipes! In micro-fluidics, however, the liquid-solid interactions have large effects. At the 
micro-scale, molecules do not come to a complete halt when they encounter a surface; in fact, they bounce around and even move opposite the direction of flow. In certain cases, if you measured the average molecular flow of a liquid along a solid, you could find that that liquid does indeed slip along the surface (the average velocity is non-zero) [1]. Slip can be a good thing. It means we can use less pressure to move liquids through pipes. It is also a parameter engineers can tune in order to produce certain types of surfaces, for example, a surface that might use slip to mix different types of liquids. An atomistically flat surface will have an intrinsic slip that is characteristic of the properties of the solid. If you combined surfaces with different intrinsic slips and even added roughness to the surfaces, would it be possible to predict some effective slip of this composite surface? Certainly one could measure the slip, but it would also be valuable to predict it given the surface characteristics. A model was recently proposed that does just this, but does it work? In Chapter 3 Section 3.3, we will test this theory using MD.

The study of small, liquid drops was initially motivated by a paper on the lotus effect [2]. This effect stands for the ability of certain plants, such as the lotus and the taro, to keep their leaves clean. These leaves exhibit hydrophobic properties, allowing water to repel from rather than stick to the surface of the leaves. One such result of these properties is the formation of liquid drops on the leaves' surfaces. How drops move on these leaves may dictate a plant's ability to self clean. It was suggested that drops which roll off leaves have a higher tendency to clean a leaf compared with drops that slide off leaves. Chapter 3 Section 3.4 will investigate the rolling and slipping motion of drops on super-hydrophobic surfaces, and it will propose a scaling law that aims to predict drop speed given drop and surface characteristics. The theory will be compared with results from MD.

The 2010 Nobel Prize in Physics was awarded to Novoselov and Geim for their groundbreaking work [3] in graphene. Graphene, a honeycomb lattice of carbon atoms only a single atom thick, is strong, flexible, transparent to visible light, and electrically conductive. It has numerous applications in a number of industries, and it will eventually replace expensive materials like indium tin-dioxide (a transparent conductor) and lead to carbon-based electronics. Manufacturing pristine graphene as well as engineering certain features into graphene are some of the hurdles to overcome before graphene is seen in mainstream products. Understanding basic properties of graphene will help scientists find better ways to manufacture graphene. In Chapter $4, \mathrm{MD}$ is used to understand the energies 
required to exfoliate graphene from graphite, and it is also used to understand the formation of nano-bubbles in graphene.

In the next Chapter, we will explain some basics on how molecular dynamics works so that the reader may have a better understanding of the simulations to follow. 



\section{Chapter 2}

\section{Molecular Dynamics}

In its simplest form, Molecular dynamics deterministically calculates a particle's trajectory using Newton's Second Law, $\mathbf{F}=m \mathbf{a}$. Particles interact with each other via forces, be it gravitational, chemical, or quantum mechanical, and MD allows scientists to use a number of force models to simulate and study physical systems.

The real value in MD, however, comes from the many tools available to a simulated system. For example, researchers can study systems at high temperatures and pressures, which may be costly compared with real experiments. MD also allows scientists to interact with simulations by moving particles or collections of particles, by applying external forces, or by adding or subtracting particles using boolean criteria. At the end of the day, a researcher's creativity will limit the number of things possible in MD.

In this thesis, MD is used to study two specific systems. This thesis does not attempt to develop any new tools, such as force models, in order to carry out its research; instead, it uses tools already developed in the literature. This Chapter will briefly cover some basic principles of MD so that the reader may understand the methods and simulations in the Chapters to follow.

\subsection{How MD Works}

Here we will briefly outline how MD works. For a proper introduction to MD, see Ercolessi [4].

The bulk properties of a system result from the molecular interactions within the system, and statistical mechanics allows us to relate the bulk, or macro state, to the molecular, or micro state. Molecular dynamics works in this way - by simulating many micro states of a system, MD can predict the bulk characteristics. 
In MD, we start with an energy potential that governs the interactions between particles. In a simple model, the potential is a function of particle position and arises from pair-wise interactions,

$$
V_{i}=\sum_{j \neq i}^{N} \phi\left(\mathbf{r}_{i, j}\right),
$$

where the total potential for particle $i, V_{i}$, is the sum of the pair-wise interactions with all other particles $j$, and $\mathbf{r}_{i, j}$ is the distance between $i$ and $j$. For this simple model and $N$ particles, there are $(N-1)$ ! pairs to consider. It would be computationally expensive to consider all pairs, so typically in MD a cut-off distance, $r_{c}$, is used where we ignore interactions for $\mathbf{r}_{i, j}>\mathbf{r}_{\mathbf{c}}$.

The force acting on particle $i$ arises from the spacial gradient of the potential,

$$
\mathbf{F}_{\mathbf{i}}=-\nabla V_{i}
$$

and with this force we can calculate the trajectory and particle position where $\mathbf{F}_{i}=m_{i} \mathbf{a}_{i}$. MD numerically integrates the equations of motion for all particles with respect to time. There is obvious room for numerical error when performing these types of integrations, so a typical algorithm used in MD that mitigates error is the velocity Verlet algorithm. The basis of this algorithm was developed by Verlet [5], where the particle position at a time $t+\Delta t$ is,

$$
\mathbf{r}_{i}(t+\Delta t)=-\mathbf{r}_{i}(t-\Delta t)+2 \mathbf{r}_{i}(t)+\mathbf{a}_{i}(t) \Delta t^{2}
$$

and $\Delta t$ is the time increment. ${ }^{1}$ The error from this algorithm is on the order of $\Delta t^{4}$, which makes it very accurate even for long simulations. The velocity Verlet algorithm improves on Eq. 2.3 by implicitly calculating particle velocities and by not depending on $t-\Delta t$. Equations $2.1-2.3$ allow MD to calculate the time evolution of the positions of particles.

The state of a simulation is simply the positions and velocities of the particles in the system at any point in time. The state of the particles will evolve until the system reaches a stable equilibrium, a point where the potential energy is at a minimum. To obtain properties of a macro state, one needs to take averages of all possible micro states for a given configuration. This is done by taking time averages, where each snap shot in time of a simulation is a representation of one of the possible micro states for a given macro state. The assumption that time

\footnotetext{
${ }^{1}$ In $\mathrm{MD}$, the time increment is called the time step
} 
averages are consistent with statistical ensemble averages is known as the ergodic hypothesis [6], and this hypothesis is the key link between MD and real systems.

\section{$2.2 \quad$ Software}

Large-scale Atomic/Molecular Massively Parallel Simulator ${ }^{2}$ or LAMMPS [7] is used in this thesis to perform classical MD calculations. LAMMPS is a freely available software package and it comes with a number of tools to help design simulations and experiments. One advantage of LAMMPS is that it can simulate large systems using many processors, as its names suggests. LAMMPS does this by dividing a simulation box into a number of grids proportional to the number of processors being used; in effect, it is using each processor to compute smaller MD simulations.

We also used the Visual Molecular Dynamics ${ }^{3}$ (VMD) package [8] in our research. VMD allows us to visualize the spacial positions of atoms in a simulation, and it is also freely available online.

\subsection{Important Considerations in MD}

Here we highlight a few aspects of MD that are important to consider when conducting a simulation.

\section{Boundary Conditions}

A common boundary condition (BC) in $\mathrm{MD}$ is the periodic boundary. This $\mathrm{BC}$ provides a good way to mimic large systems by only considering a smaller portion. When a particle moves across a periodic boundary, it is mapped back into the simulation box opposite the boundary. When using periodic boundaries, it is important that the box dimensions are consistent with the periodicity of the structure (e.g. a lattice) and that the simulation box is bigger than any characteristic length scales of the system (e.g. the cut-off radius of a potential or the size of a unit cell). The other common BC is the fixed BC. In this instance atoms are not mapped back into the simulation box, but they can be reflected or even ignored depending on the type of system you wish to create.

\footnotetext{
${ }^{2}$ http://lammps.sandia.gov/.

${ }^{3}$ http://www.ks.uiuc.edu/Research/vmd/.
} 


\section{Thermostatting}

Proper management of the temperature of a simulation is very important. For example, you may want to study the interaction of a liquid with a surface. To improve the efficiency of your simulation and decrease computation time, you can keep the surface fixed and simply compute the trajectories of the liquid. Unfortunately, this means that the temperature of your surface is zero and the liquid will lose heat to the surface. To overcome this, you can artificially manage the temperature of the liquid, and there are a number of methods to do this. The velocity of atoms determines their temperature, and MD simply calculates temperature by adding up the velocities of a system. If, for example, you have a moving liquid, it may appear hotter as the flow velocity will affect the temperature calculation. One could bias this calculation by removing flow energy in order to calculate the proper liquid temperature.

\section{Data Collection}

To get good data, one has to take sufficient averages until the estimates converge to their expected value. This includes time averages and spacial average. Let's say you wanted to look at the velocity of liquid along the axis orthogonal to the flow. First, you should find spacial averages of the particle velocities along the axis. These spacial averages give you a representation of the actual speed of the liquid at a point in space rather than considering the speed of every single atom. Spacial averaging is usually referred to as binning, where atoms are placed into spacial boxes and their averaged characteristics are recorded (e.g. velocity, density, energy). These spacial averages give you a representation of the flow profile at an instance of time. Then, by taking time averages over sufficient amounts time, the spacial averages will converge to the expected flow profile of the liquid. The reliance on time averages to produce thermodynamic quantities is a primary reason why an accurate time integration algorithm is required. 


\section{Chapter 3}

\section{Modeling Liquids}

The ability to study fluids is aided by the fact that fluids obey scaling laws; that is, one can build a model sized propeller, study how water flows through the propeller, and scale the data up to predict precisely how the real system will perform. Likewise, the results from studies of fluids in MD on small systems are easily scalable to real systems. Although the particle spacing (mean free path) in an MD model may be vastly different than the spacing in a real-world liquid, the MD models often predict real properties of fluids with good accuracy.

In this Chapter, we use the well studied Lennard-Jones (L-J) potential to model a liquid. L-J liquids are scalable and provide accurate descriptions of real liquids. Furthermore, the properties of L-J liquids, given a set of parameters, are readily available in the literature.

First we demonstrate how to calculate various properties of L-J liquids in Section 3.2. Then, in Section 3.3 we study slip at the liquid-surface interface and demonstrate how effective slip is dependent on the intrinsic slips and geometries of a surface. Finally, we will study the dynamics of liquid drops moving on superhydrophobic surfaces in Section 3.4, and we will propose a theory that will predict the behavior of drop speed.

\subsection{The Lennard-Jones Liquid}

The Lennard-Jones (L-J) 6-12 potential provides a reasonable representation for the interaction between atoms,

$$
V(r)=4 \epsilon\left[\left(\frac{\sigma}{r}\right)^{12}-\left(\frac{\sigma}{r}\right)^{6}\right] .
$$


Here, the depth of the well is $\epsilon$, the characteristic length between two atoms is $\sigma$, where $\sigma$ is the zero-potential distance and $2^{1 / 6} \sigma$ is the equilibrium distance, and the distance between two atoms is $r . r^{-12}$ is the repulsive term of the potential and $r^{-6}$ is the attractive term. The simplicity of the L-J potential makes it computationally fast, and it allows the researcher to explore the parameter space by changing only a few values. The L-J potential also provides an accurate description of noble elements, and Argon is often used as a model to compare results from MD with experiment.

For the simulations to follow, we work in reduced L-J units, where all quantities are dimensionless. This is achieved by setting the fundamental quantities $\epsilon$, $\sigma$, mass $m$, and the Boltzmann constant $k_{B}$ to 1 . This implies that the following dimensionless quantities, denoted by an asterisk, are as follows,

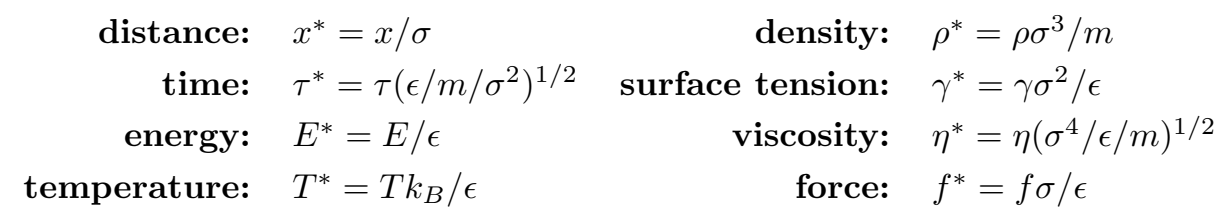

To convert between dimensionless and dimensional quantities, you choose values of $\epsilon, \sigma$, and $m$ for a particular material and use those values in the equations above. In LAMMPS, when the user specifies values for $\epsilon, \sigma$, and $m$, those values are multiples of the fundamental quantities. For example, we will study a liquid with $\epsilon_{l l}=1.75$, where $l l$ denotes liquid-liquid interactions. This implies that the energies in our system will be higher, $1.75 E^{*}$, and for some value of $\epsilon$, our model is the equivalent of a system with $1.75 \epsilon$.

A typical cutoff of $2.5 \sigma$ is used in our simulations. This value provides accurate atomistic descriptions as well as reasonable computation speeds. There are a number of papers that look into the effects of the cutoff radius, and it is well know that changing the cutoff will certainly change the phase diagram of a system $[9,10]$. Fortunately, this only means that we must use the same cutoff distance throughout our simulations so that our calculated properties are consistent and relevant to our system. Finally, all boundary conditions used in this Chapter are periodic unless otherwise noted.

\subsection{Liquid Properties}

The L-J parameters and temperature for the liquid used in this Chapter are provided in Table 3.1 in addition to calculated values for density, surface tension, and viscosity. These parameters were chosen because the theory in Section 3.4 


\begin{tabular}{|lcl||lll|}
\hline Parameters & & & Properties & & \\
\hline Potential & $\epsilon$ & 1.75 & Density & $\rho^{*}$ & 0.85 \\
Distance & $\sigma$ & 1 & Surface Tension & $\gamma^{*}$ & 1.6 \\
Temperature & $T^{*}$ & $1 \pm 0.02$ & Viscosity & $\eta^{*}$ & 3.5 \\
\hline
\end{tabular}

Table 3.1: Liquid parameters and properties.

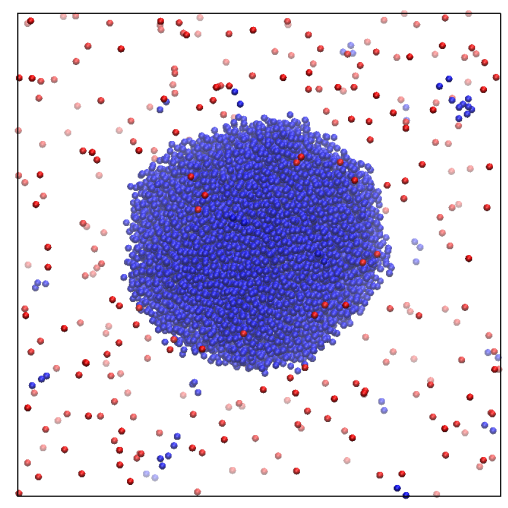

Figure 3.1: A Lennard-Jones liquid. A snap shot of a liquid, L-J drop shows the existence of both vapor (red) and liquid (blue) phases, a consequence of the L-J potential.

requires liquids with high viscosity. These three parameters alone define the characteristics of a L-J liquid.

There are a few challenges in obtaining good data in order to calculate some properties of a L-J liquid. First off, a simulation of a L-J liquid will contain a liquid phase and a vapor phase, as demonstrated in Figure 3.1. During a simulation, a vapor atom may condense onto the liquid and vise versa. These vapor atoms must be removed or ignored when estimating properties such as atom energies. Secondly, when considering a liquid drop, the shape and size of a drop will fluctuate, and this makes finding a drop's radius tricky. Both fluctuations in drop energies and radius will make calculations of various liquid properties difficult. By performing simulations with large amounts of atoms and over a sufficient number of time steps, the predictions of the liquid properties should converge.

The following sections will demonstrate how to separate liquid from vapor and how to calculate density, surface tension, and viscosity for a L-J liquid. Details of the simulations used for these calculations are provided in Appendix A.

\subsubsection{Separating Liquid from Vapor}

The simplest method to separate a L-J vapor from a L-J liquid is to look at the potential energies of the atoms. All atoms with zero potential energy are 
beyond the cutoff radius of the L-J potential and are therefore not a part of the liquid; still, atoms in the vapor phase will interact with other vapor atoms thereby creating a non-zero potential. One could create a potential energy threshold, $V_{t}$, such that all atoms that do not meet this threshold are considered vapor atoms, but caution must be taken for atoms near the surface of the liquid which may not meet such criteria even though they may be a part of the liquid.

Our technique used to separate vapor from liquid consists of two parts. First, an energy threshold is chosen by considering the largest potential energy an atom would have at the surface of a liquid. For the case of the L-J potential with a cutoff of $2.5 \sigma$ and assuming a simple cubic structure, the highest potential energy is $5 \mathrm{~V}(2.5)$, where an atom on the surface of the drop has 5 nearest neighbors and the L-J potential, $V$, is evaluated at the cutoff radius. For the liquid chosen here, $V_{t}^{*}=-0.143$.

The next step is to look at the location of the atoms. Visual inspection is a simple method to decide which atoms do not belong to the liquid drop. The computational equivalent to this is to look at each atom's distance from other atoms, particularly those atoms pertaining to the liquid; however, at worst this computation requires $N^{N}$ computations for $N$ number of atoms. ${ }^{1}$ Instead, we will create an ordered list of the radial distances from the center of mass of all atoms in the simulation box. The center of mass of the drop is the average location of all atoms in the simulation, including vapor atoms that don't meet the energy threshold since they have a negligible effect.

If the simulation contained no vapor atoms and the drop were a perfect sphere, then the radius at $R_{n}$ would simply be related to the volume of a sphere, where $n=0$ and $n=N$ are the atoms closest to and furthest from the center of mass, ${ }^{2}$ such that,

$$
R_{n}=\left(\frac{3}{4 \pi} \frac{n}{\rho}\right)^{1 / 3} .
$$

Now consider $n \gg 1$, then the change in radius, $\Delta R_{n}$, from one atom to the next within the drop is $R_{n}-R_{n-1} \approx 0$. Therefore, atom $n$ is certainly not a part of the liquid drop if $\Delta R_{n} \gg 0$. Assuming the liquid drop is relatively spherical, we can find the first instance where $\Delta R_{n} \gg 0$ and reject all atoms from $n$ and above. Figure 3.2 shows the behavior of this relationship for a set of data (blue-). Notice that for atoms with radii larger than $5, \Delta R^{*}$ is nearly zero. Then at around

\footnotetext{
${ }^{1}$ LAMMPS avoids large amounts of computations by using a cut-off distance to generate a list of atom neighbors. Using this neighbor list may provide an efficient way to determine the phase of an atom on-the-fly, but that list may not be readily available through the LAMMPS software.

${ }^{2} R_{n}$ is an ordered list of the radial positions of all atoms from the center of mass.
} 
$R^{*}=15, \Delta R^{*}$ increases and fluctuates, indicating that the radius of this drop is around 15. The requirement that $\Delta R_{n}$ be large is ambiguous when considering Figure 3.2, and atoms near the center of the drop meet this criteria though they are definitely a part of the liquid. To alleviate this uncertainty, we bias the data by multiplying $\Delta R$ by $R$. Figure 3.2 (red - ) shows that fluctuations in the vapor phase are larger than unity, even close to the liquid. We could therefore define our drop radius as the first instance where $R_{n} \Delta R_{n}>1$. This criterion is similar to looking at the change in surface area between spherical shells. The choice of unity may still be an ambiguous requirement, but this criterion worked very well for the viscous liquid chosen here. It is possible that unity arises from our $\sigma$ parameter in the L-J potential, and it would make sense that a larger value of $\sigma$ would increase $\Delta R$ since the characteristic distance between particles is $\sigma$.

The downside of using a radial cutoff is that it may include vapor and exclude liquid atoms near the surface of the drop for instances where the drop diverges from its spherical shape; but, this method will provide a fast and good approximation for separating liquid and vapor. Furthermore, taking time averages will increase the accuracy of this method. Figure 3.3 provides an example of this routine in action.

Another method to find the radius is to simply use the number of atoms and the densities [11],

$$
N=\rho_{l} \frac{4}{3} \pi R_{e}^{3}+\rho_{v}\left(L^{3}-\frac{4}{3} \pi R_{e}^{3}\right),
$$

where $\rho_{v}$ and $\rho_{l}$ are the vapor and liquid densities, $L^{3}$ is the simulation box volume, and $R_{e}$ is the equimolar radius of the drop. $R_{e}$ is the radius which divides the liquid and the vapor assuming that the size of this liquid-solid interface is nil. For a L-J liquid, this may not be the case and this method will result radii smaller than our method above. This smaller cutoff radius will exclude liquid atoms and may possibly lead to errors in estimating energies. For our purposes, we use our method as the way in which we calculate surface tension requires accurate estimates of drop energy.

\subsubsection{Density}

Having successfully isolated a liquid drop, the simplest method to calculate density is to use Equation 3.2 and to fit this curve to the data for $R_{n}$. To improve the quality of the fit, we can exclude atoms for small values of $n$ and exclude atoms near the surface of the drop. If we included these atoms, we would find a decrease in the density because the atom count near the center is too small to give 


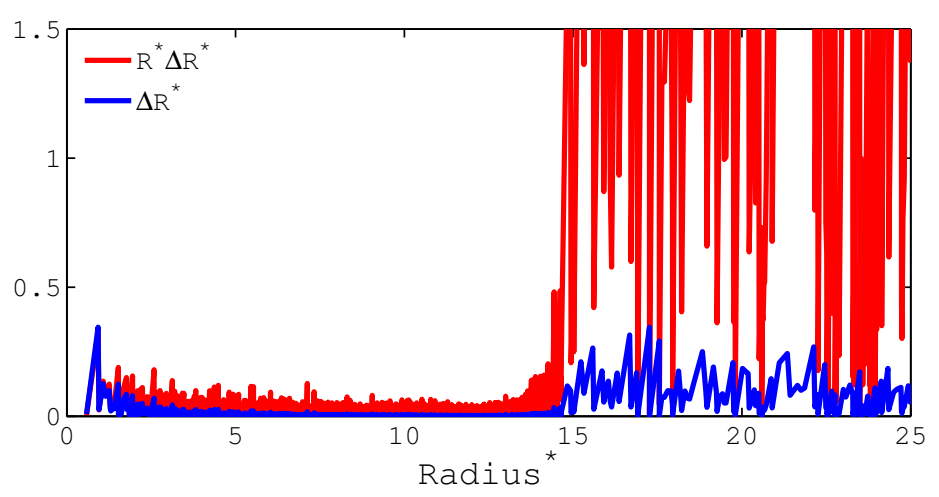

Figure 3.2: Radial behavior of drop atoms. The change in radius, $\Delta R$, is plotted as a function of $R$ (blue). $\Delta R^{*} \approx 0$ within the drop except near the center and near the surface. The graph indicates that $\Delta R^{*} \gg 0$ near $R^{*}=15$. Biasing the data by $R$ (red) provides a higher certainty that the drop's radius is approximately 15 because $R \Delta R$ is typically greater than unity in the vapor region.

a reasonable estimate of the density and atoms near the surface of a L-J liquid tend to evaporate. For our purposes, we considered data where $0.1 N<n<0.5 N$ to ensure that we were within a well behaved region of the drop. We calculated the density for drops over a large number of sizes, and we averaged the data over large time steps. Calculated densities are provided in Table A.1, and we used an average of the density over all simulation sizes, such that $\rho^{*}=0.85$.

\subsubsection{Surface Tension}

Finding the surface tension of a liquid drop is not trivial. Many methods require finding the radius of the drop, which is not necessarily clear and consistent for a L-J liquid. Surface tension, $\gamma$, as defined by Laplace, is proportional to the difference in pressure between the liquid and the vapor,

$$
\Delta P=\frac{\gamma}{R}
$$

We could use Equation 3.4 to find $\gamma$, but our estimate of $R$ will also affect our estimate of $\Delta P$ since this pressure is dependent on the volume of the liquid and the vapor. Another method to find $\gamma$ relates a drop size to its energy [12],

$$
V=A N+B N^{2 / 3}+C N^{1 / 3}+D
$$

where $V$ is the potential energy of the drop, $N$ is the number of atoms in the drop, $A N$ is a bulk binding energy, $B N^{2 / 3}$ is the surface energy contribution, and $C N^{1 / 3}$ and $D$ account for non-sphericity. Since our drops are relatively spherical, $C$ and $D$ will be negligible, and surface tension can be approximated as, 

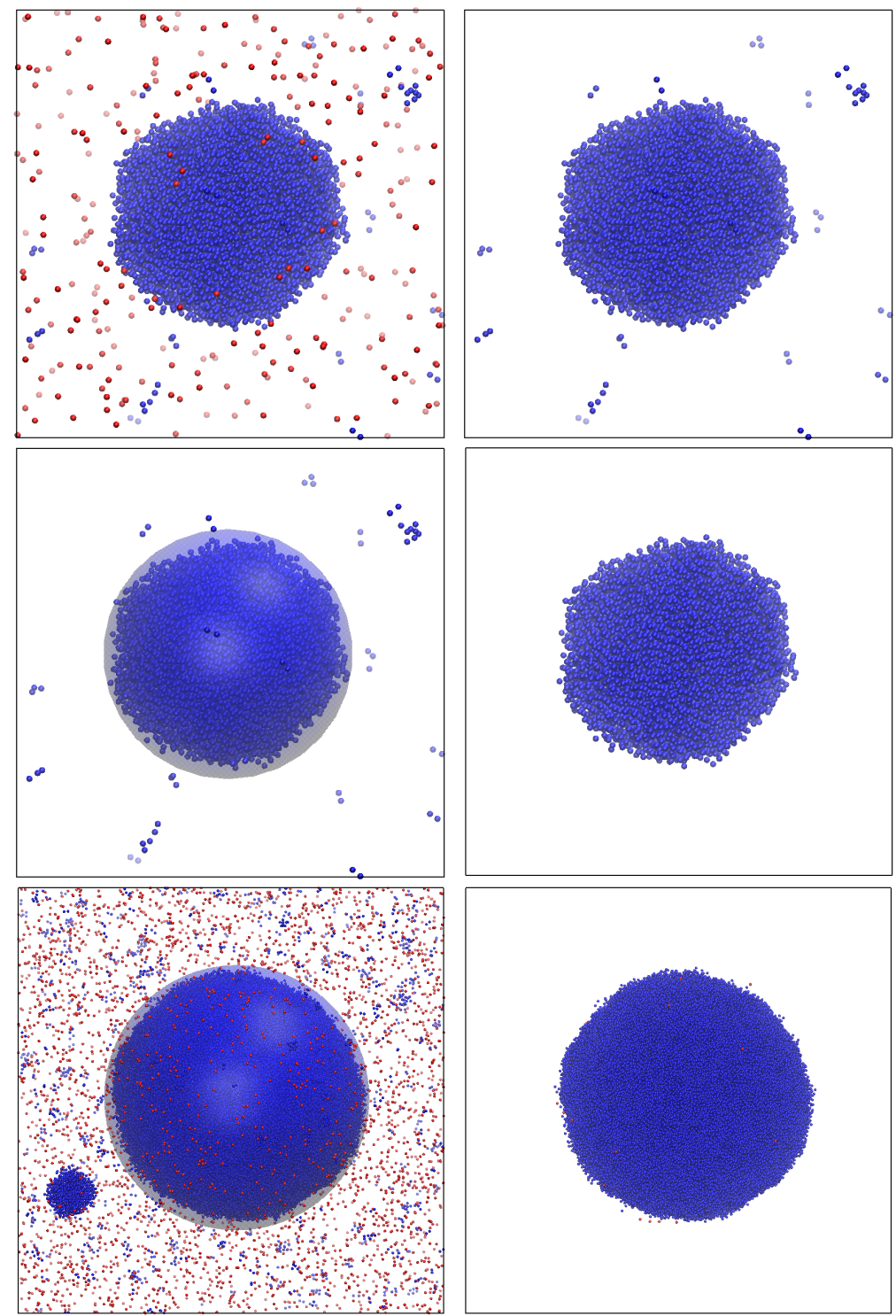

Figure 3.3: Method for separating vapor from liquid. Vapor atoms are initially selected using a potential energy threshold (red, top left), but some clumps of vapor atoms remain as they have potential energies larger than the threshold (top right). Next, a radial cutoff is calculated by looking at the differential change in each atom's radial position (middle left). Finally, a liquid drop is isolated from the vapor phase (middle right). This method works well for large simulations, even when smaller drops may coalesce in the vapor region (bottom). 


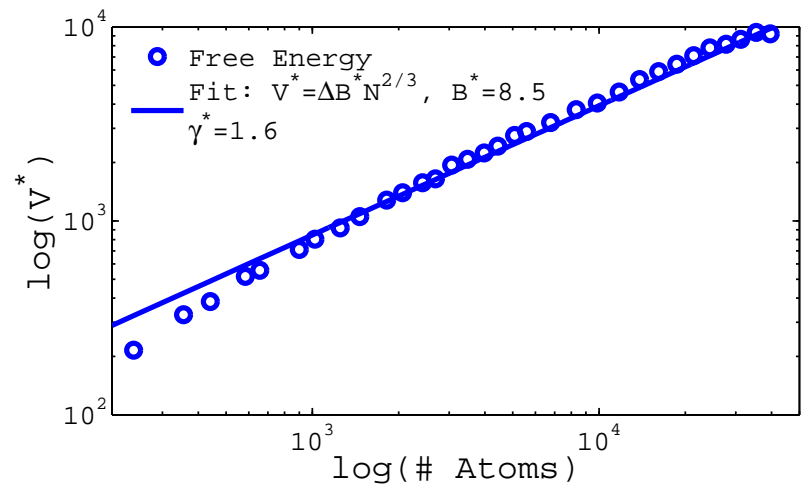

Figure 3.4: Potential energy of a drop versus drop size. A drop's potential energy has a surface energy contribution proportional to $B N^{2 / 3}$. Here, we plot $\log (\Delta B)$ versus the $\log$ of the number of atoms. The data (o) agree very well with the relationship $V=\Delta B N^{2 / 3}$ $(-)$. Fitting this relationship to the data provides an estimate of the surface tension. Note the convergence of $\Delta B$ at large $N$.

$$
\gamma=\frac{\Delta B N^{2 / 3}}{4 \pi R^{2}}
$$

where $R$ is the radius of a drop of $N$ atoms, and $\Delta B$ is the free surface energy. Obtaining the potential energy of the drop is straight forward. To find $\Delta B$, we recalculate the potential of the drop after removing the vapor phase. This recalculated potential represents the bulk liquid energies exclusive of the vapor regime near the drop's surface. The difference between the energies of a drop with the vapor phase and the same drop without the vapor phase represents the additional surface energy that gives the drop its shape. To improve our calculation of $\gamma$, we substitute $N$ for $R$ using our calculated density, such that $\gamma=\Delta B(4 \pi)^{-1 / 3}(\rho / 3)^{2 / 3}$.

Figure 3.4 shows the dependency of $\Delta B$ on the number of atoms. We fit the data to the curve $V=\Delta B N^{2 / 3}$, and the data (o) fit this curve very well (-) especially as $N$ increases. Given $\Delta B^{*}=8.5$, we find $\gamma^{*}=1.6$. Values for $\gamma$ in the literature are primarily for systems where $\epsilon_{l l}=1$, in which case $\gamma \approx 0.4$ [13]. The state of our system with $\epsilon_{l l}=1.75$ and $T^{*}=1$ is equivalent to a system where $\epsilon_{l l}=1$ and $T^{*}=1 / 1.75=0.57$. The surface tension of Argon as a function of temperature is,

$$
\gamma=\gamma_{0}\left(1-T / T_{c}\right)^{p}
$$

where $p=1.281, T_{c}=150.7 \mathrm{~K}$, and $\gamma_{0}=2.376 \mathrm{meV} / \AA^{2}[14]$. For Ar where $\epsilon=$ $10.03 \mathrm{meV}$ and $\sigma=3.370 \AA$, the dimensionless temperatures scale to $T=66.52 \mathrm{~K}$ for $\epsilon_{l l}=1.75$ and $T=116.4 \mathrm{~K}$ for $\epsilon_{l l}=1$. Using Eq. 3.7 and scaling back to dimensionless units, we find $\gamma^{*}\left(\epsilon_{l l}=1\right)=0.404$, consistent with experiments and 
MD simulations, and we find $\gamma^{*}\left(\epsilon_{l l}=1.75\right)=1.276$. This value is in reasonable agreement with our calculated value. We will use 1.6 in our analyses to follow.

\subsubsection{Viscosity}

There are a few ways to calculate a liquid's viscosity in MD [15]. Here, the Green-Kubo equation is used,

$$
\eta=\frac{V_{L}}{k_{B} T} \int_{0}^{\infty}<P_{i j}\left(t_{0}\right) P_{i j}\left(t_{0}+t\right)>_{t_{0}} d t
$$

where $\eta$ is the viscosity, $V_{L}$ is the volume, $k_{B}$ is the Boltzmann constant, $T$ is temperature, and $P_{i j}$ is the off-diagonal term of the pressure tensor for $i j=x y, x z, y z$. Code from the work of Schebarchov [16] was used to compute the autocorrelation of the pressure tensors as well as the integral of the autocorrelation function. The computed result for viscosity, $\eta=3.5$, is similar to that of other work with similar density and temperature $(\eta \approx 3)[17-19]$. 



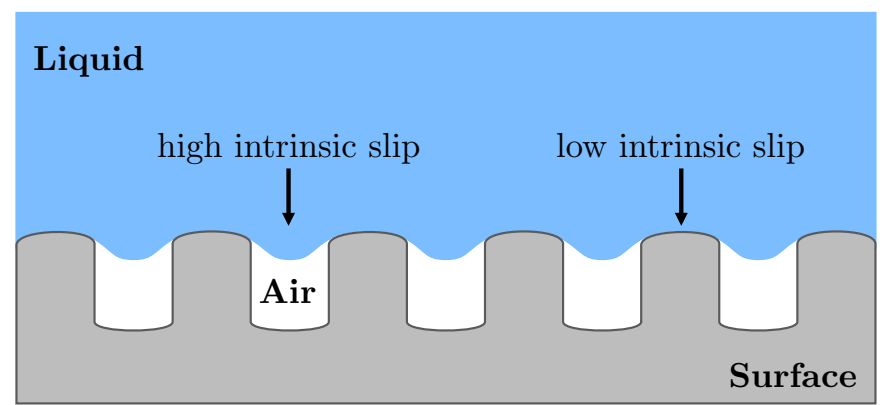

Figure 3.5: A super hydrophobic surface with posts. The intrinsic slip between a liquid and a surface (blue and gray) is low compared to the intrinsic slip between a liquid and a vapor (blue and white). The effect of these two intrinsic slips give rise to an effective slip for the surface.

\subsection{Estimating Slip of Mixed Surfaces}

In this section, we estimate the slip length of a substrate that contains regions of large slip and low slip. This type of system is analogous to a hydrophobic leaf with a rough, waxy surface. The wax provides the hydrophobic properties (the lower slip region) and the surface roughness creates areas where liquid does not touch the leaf but rather touches a small layer of vapor that separates the liquid from the leaf (a region of high slip). Figure 3.5 provides an example of this type of surface. The liquid (blue) only touches a fraction of the surface (gray) while pockets of air (white) help to give the surface its super-hydrophobicity.

The intrinsic slip is a slip that arises from the molecular interactions between two substances, and in Figure 3.5 the interactions between the liquid and the surface will have a lower intrinsic slip than the interactions between the air and the liquid. From a macroscopic view, however, the surface in this Figure may appear smooth; in fact, if we measure the slip between the liquid and the surface, then we would measure an effective slip length. This effective slip length arises from the combination of intrinsic slip lengths.

Nat Lund et al. have proposed a theory that aims to calculate the effective slip of a surface given the geometry and the intrinsic slips [20]. We will use MD in this Section to test the validity of this theory.

\subsubsection{Theory}

\section{The slip boundary condition}

The no-slip boundary condition states that the fluid at a liquid-solid interface moves at the same speed as the solid. In most situations and on a macro-scale, this boundary condition provides an accurate description of the interaction; however, 

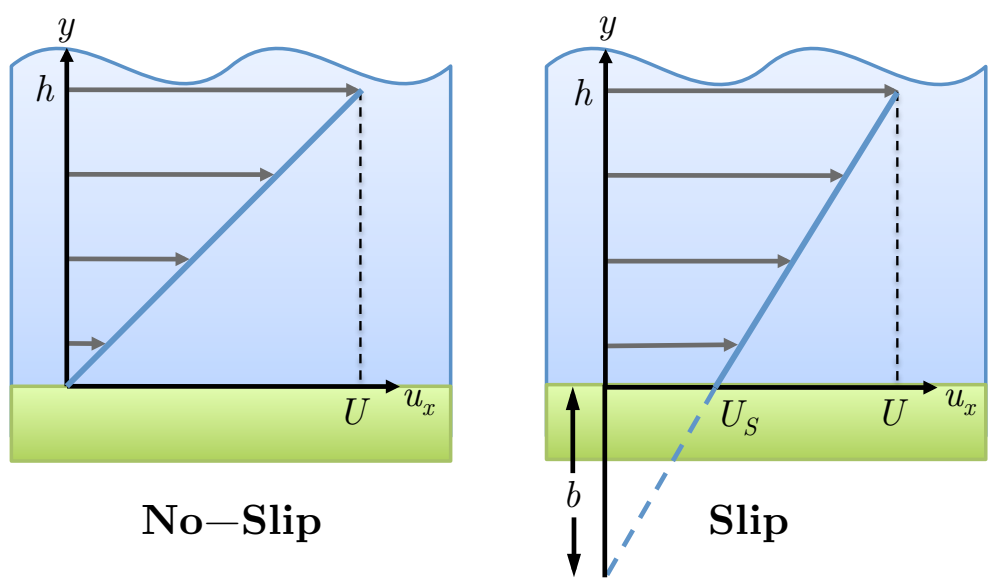

Figure 3.6: No-slip versus slip. If the top of a liquid moves at a constant speed along the $\mathrm{x}$-direction, then the speed of the liquid at the liquid-surface interface will be zero (no-slip) or $U_{s}$ (slip), and the gradient of the liquid's velocity, $\partial u_{x} / \partial y$, will be $U / h$ (no-slip) or $\left(U-U_{s}\right) / h$ (slip). $b$ is the slip length.

at the micro and nano scales, molecules and atoms of a liquid may not necessarily share the same speed as the solid.

Slip is usually characterized by a slip length. This slip length, $b$, arises from the Navier slip boundary condition, which states that the tangential velocity of a liquid at the liquid-solid boundary is proportional to the shear rate at the surface [21],

$$
U_{s}=b \frac{\partial u}{\partial y}
$$

where $\partial u / \partial y$ is the shear rate normal to the surface and $U_{s}$ is the speed of the liquid at the liquid-surface interface (slip speed). Figure 3.6 demonstrates the comparison between no-slip and slip. Assuming a Couette flow where the top of the liquid (blue) moves at a constant speed parallel to the surface (green), the change in speed of the liquid along the height of the liquid is constant. In the case of no-slip, that gradient is $U / h$ where $U$ is the speed of the liquid at the top and $h$ is the liquid height. In the case of slip, the gradient is $\left(U-U_{s}\right) / h$, and together with Eq. 3.9, this is equivalent to $U /(h-b)$. Slip length is sometimes refered to as an imaginary length below a surface where an extrapolated slip speed is zero. If we consider the slip case in Fig. 3.6 along with $U /(h-b)$, then we can see from where this "imaginary length" arises. Note that a slip length of zero is equivalent to no-slip as $U_{s}$ is zero. If $b=\infty$, then there is perfect slip since this implies that $\partial u / \partial y=0$. Perfect slip is a case where the speed in the liquid is constant along the liquid's height in the case of a Couette flow. 


\section{Effective slip}

Consider Figure 3.5 where the intrinsic slip lengths are $b_{\text {surf }}$ and $b_{\text {air }}$. The area fractions of the liquid touching the air and surface are $\beta_{\text {air }}$ and $\beta_{\text {surf }}$. The normalized arc lengths of the surface and the $\operatorname{air}^{3}$ are $s_{\text {surf }}$ and $s_{\text {air }}$, where the normalization is taken over the interval of the arc. With these parameters, the effective slip length is shown to be [20],

$$
b_{\mathrm{eff}}=\left[\frac{\beta_{\mathrm{air}} s_{\mathrm{air}}}{b_{\mathrm{air}}}+\frac{\beta_{\mathrm{surf}} s_{\mathrm{surf}}}{b_{\mathrm{surf}}}\right]^{-1} .
$$

This theory predicts $b_{\text {eff }}$ using weak convergence methods for partial differential equations. The Navier-Stokes equations along with their intrinsic slip boundary conditions (e.g. $b_{\text {air }}$ and $b_{\text {surf }}$ ) and geometries are solved to find $b_{\text {eff }}$.

\subsubsection{Simulation}

\section{Surfaces}

The intrinsic and effective slip lengths are estimated for three homogenous surfaces and five binary surfaces, respectively, as shown in Figure 3.7. The color coding in this Figure represents the value of the liquid-surface interaction parameter $\epsilon_{l s}$. The green surface in $\mathrm{A}$ is used for the outer ends of the surfaces in $\mathrm{D}-\mathrm{H}$. The cyan surfaces in $\mathrm{B}$ and $\mathrm{C}$ have the same energy parameter, and they are used for the center of the surfaces in D-H. All surfaces have a simple cubic lattice structures with a lattice parameter of $a=1$. The surface in $\mathrm{C}$ has the (111) lattice plane normal to the y-axis and all other surfaces have the (010) plane normal to the y-axis. Surfaces D-H are combinations of surfaces A-C, where surface $\mathrm{C}$ represents the steps (or cuts) in the lattice of surfaces E-F. Details of the dimensions of the surfaces and the lattice planes are provided at the bottom of Figure 3.7. All surfaces share the same dimensions except for a variation in depth for surfaces E-H. The 3-atom depth of the surfaces is sufficient for a radial cutoff of $r_{c}=2.5$. Since surfaces A-C are atomically flat, estimates of the slip length for these surfaces will give us the intrinsic slip. All other estimates will be of the effective slip length.

\footnotetext{
${ }^{3}$ The arc length of the air is the arc length of the liquid suspended over the air
} 


\section{A}

\section{B}

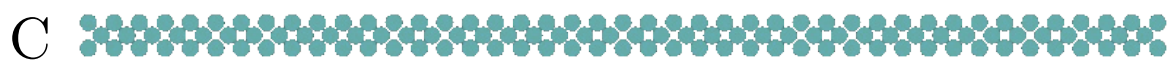
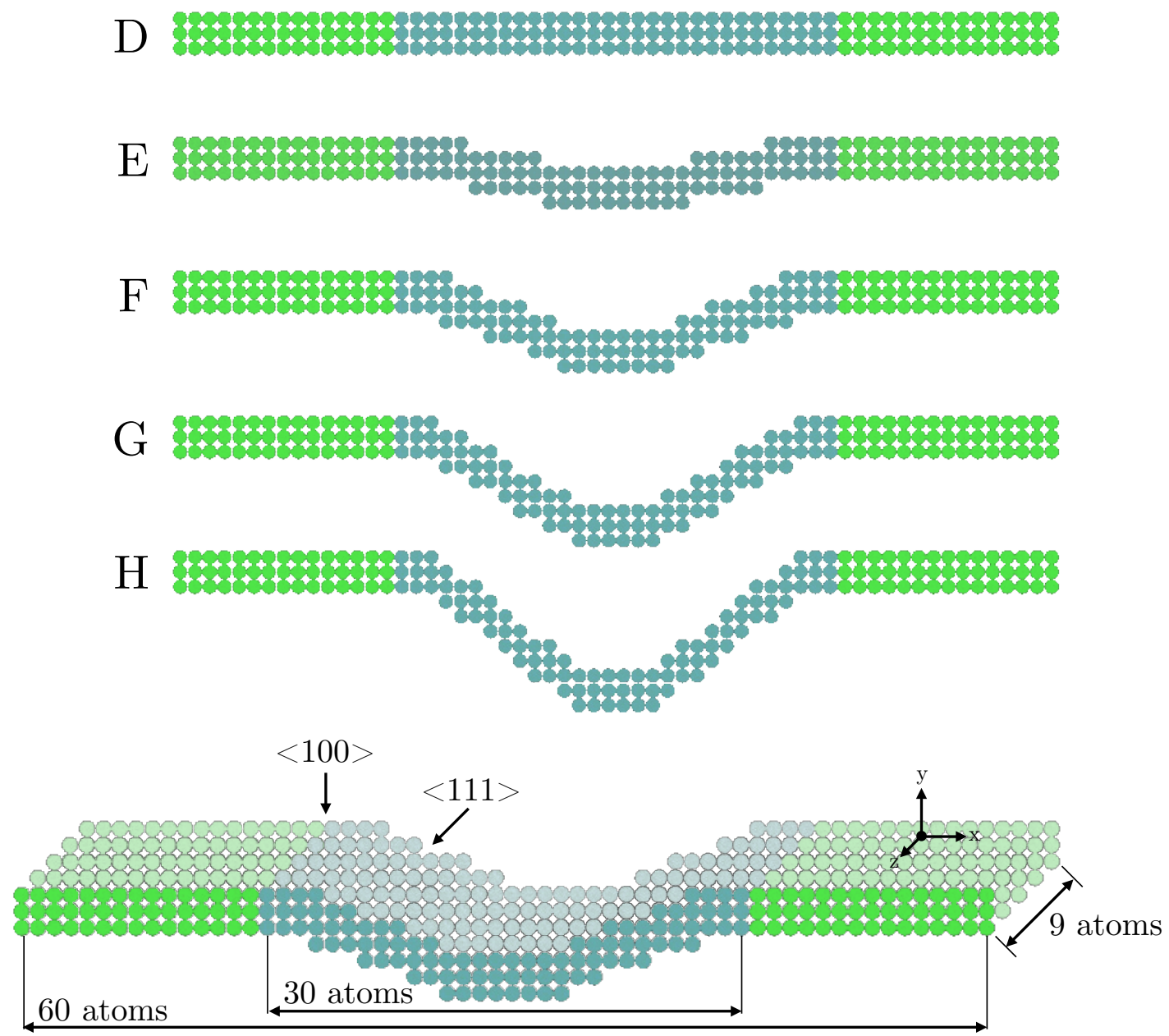

Figure 3.7: Slip surfaces. The above surfaces are of a simple cubic lattice structure with lattice parameter $a=1 \sigma$. Surfaces A and B are used to estimate the intrinsic slip lengths of flat surfaces with different liquid-solid interaction parameters. Green represents one value for the liquid-solid interaction parameter, $\epsilon_{l s}$, and cyan represents a different value. Surface C is used to estimate the slip length along the (111) lattice plane as this surface represents the steps (or cuts) of surface B in surfaces E-H. Surfaces D-H are used to estimate the effective slip lengths of a mixture of surfaces $\mathrm{A}-\mathrm{C}$, and the ratio of the mix varies for $\mathrm{D}-\mathrm{H}$, with $\mathrm{D}$ containing none of $\mathrm{B}$ and $\mathrm{H}$ containing the most of $\mathrm{C}$. The bottom of the Figure provides the dimensions of the surfaces, which are the same for all except for a varying depth in E-H. Note that since the L-J radial cut off is $2.5 \sigma$, the depth of these surfaces (3) is sufficient for our simulations. These surfaces are periodic in $x$ and $z$. 


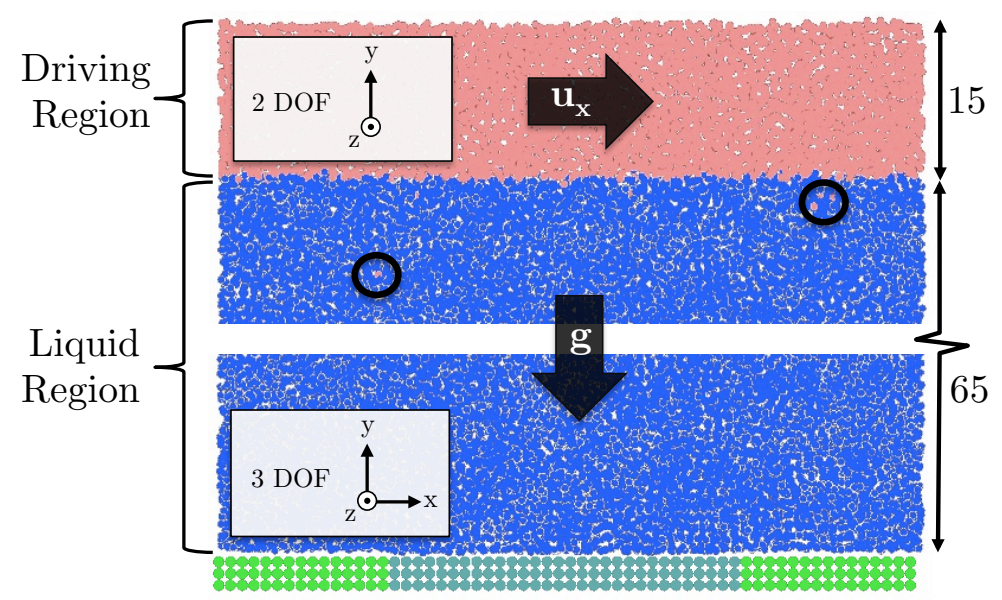

Figure 3.8: Simulation set up. All atoms in the liquid and the driving regions feel an acceleration of $g$. Atoms in the driving region move at a constant speed of $u_{x}$ and have 2 degrees of freedom $(\mathrm{y}, \mathrm{z})$, and atoms in the liquid region have 3 degrees of freedom ( $\mathrm{x}, \mathrm{y}$, $\mathrm{z})$. These simulations were periodic in $x$ and $z$ and fixed in $y$. Notice that some atoms from the driving region enter the liquid region during a simulation (black circles). This effect is negligible to the Couette flow of the system, and we update the driving region atoms every $100 \tau$ to accommodate for this effect. The simulation box was $60 \mathrm{x} 100 \mathrm{x} 9$ (xyz) atom lengths in size, periodic in $\mathrm{x}$ and $\mathrm{z}$, and fixed in $\mathrm{y}$. The time step was $1 \times 10^{-3}$, and a thermostat was used in the z-direction only with $T^{*}=1 \pm 0.1$

\section{Estimating Slip}

Both slip speed and shear rate are measurable quantities of a fluid flowing over a substrate, and by measuring these we can estimate the slip length of a surface. For the case of a smooth, homogenous surface, there will only exist one inherent slip length. Any estimate of a slip length between a liquid and this surface will be an estimate of the intrinsic slip. For the case of a surface with more than one intrinsic slip and with some surface roughness, any estimate of slip length will be the effective slip length.

To estimate the slip length, we will set up a Couette flow in our simulation so that we may produce velocity profiles similar to those in Figure 3.6. In order to do this we move the top portion of our liquid at a constant speed, and we also move the liquid slow enough so that we have laminar flow, especially near the liquid-surface boundary.

A typical method to create a Couette flow in MD is to move a driving plate (a solid surface) along the top of a liquid; however, if the liquid has a high viscosity, the driving plate could potentially cause the liquid to freeze depending on the system parameters. This occurs because the solid surface creates local ordering ${ }^{4}$

\footnotetext{
${ }^{4}$ Local ordering is when atoms in a liquid begin to arrange in the lattice structure of the solid with which it interacts. When liquid atoms assume a lattice structure, they freeze and become a solid.
} 
in the liquid near the surface, and that ordering can cause all liquid atoms to freeze.

We developed a different method to move our viscous liquid. Rather than moving a solid plate, we moved the liquid at the top at a constant speed. A diagram of this set up is shown in Figure 3.8. The liquid at the top is denoted as the driving region. ${ }^{5}$ Atoms in the driving region were fixed in the $\mathrm{x}$-plane and allowed to move in the $\mathrm{z}$ and $\mathrm{y}$-planes. The degrees of freedom for an atom are decreased when preventing it from moving in a certain direction, and this loss of freedom implies that an atom is "frozen" in that dimension. By allowing atoms to move in $\mathrm{y}$ and $\mathrm{z}$, we prevent the driving region from creating order in the liquid region. This freedom also meant that some atoms in the top region moved along the y-axis and into the liquid region (see the circles in Figure 3.8), so atoms in the driving region changed over time. To accommodate for this brownian motion, the driving region was updated every $1 \times 10^{5}$ time steps to include a new set of atoms at the top of the liquid.

The soft boundary conditions used in our method required a force to prevent the liquid from floating away from the surface, so we added a gravity component acting normal to the surface. This gravity creates a pressure which will affect the estimated slip length [1], and increasing pressure also increases the ordering of liquid near the surface. We used a small enough force so as to prevent additional ordering of the liquid, and we used the same force throughout all simulations on surfaces A-H for consistency.

We will use Equation 3.9 to calculate the slip length by fitting a line to the velocity profiles of the liquid. This profile is obtained by recording the velocity of the liquid along the height of the liquid so that we produce the curve $y=u_{x}(y)$. This creates a Couette profile like that in Figure 3.6. The y-intercept of the fitted curve provides an estimate for the slip length.

\subsubsection{Results}

We conducted three sets of simulations (each set called a Run) to estimate the slip lengths of our surfaces while varying gravity as well as the liquid-surface interaction. Results from these simulations are presented in Figure 3.9, and details of simulation parameters can be found in Appendix B. The graphs in Figure 3.9 show the slip lengths for the primary surfaces A-C (--) and surfaces D-H $(\bullet)$. The left and center graphs (blue and red, respectively) represent simulations with $g^{*}=0.05$ while the right graph (green) represents a simulation with $g^{*}=$

\footnotetext{
${ }^{5}$ Atoms in the driving region are liquid atoms with the same $\epsilon_{l s}$ as the other liquid atoms.
} 

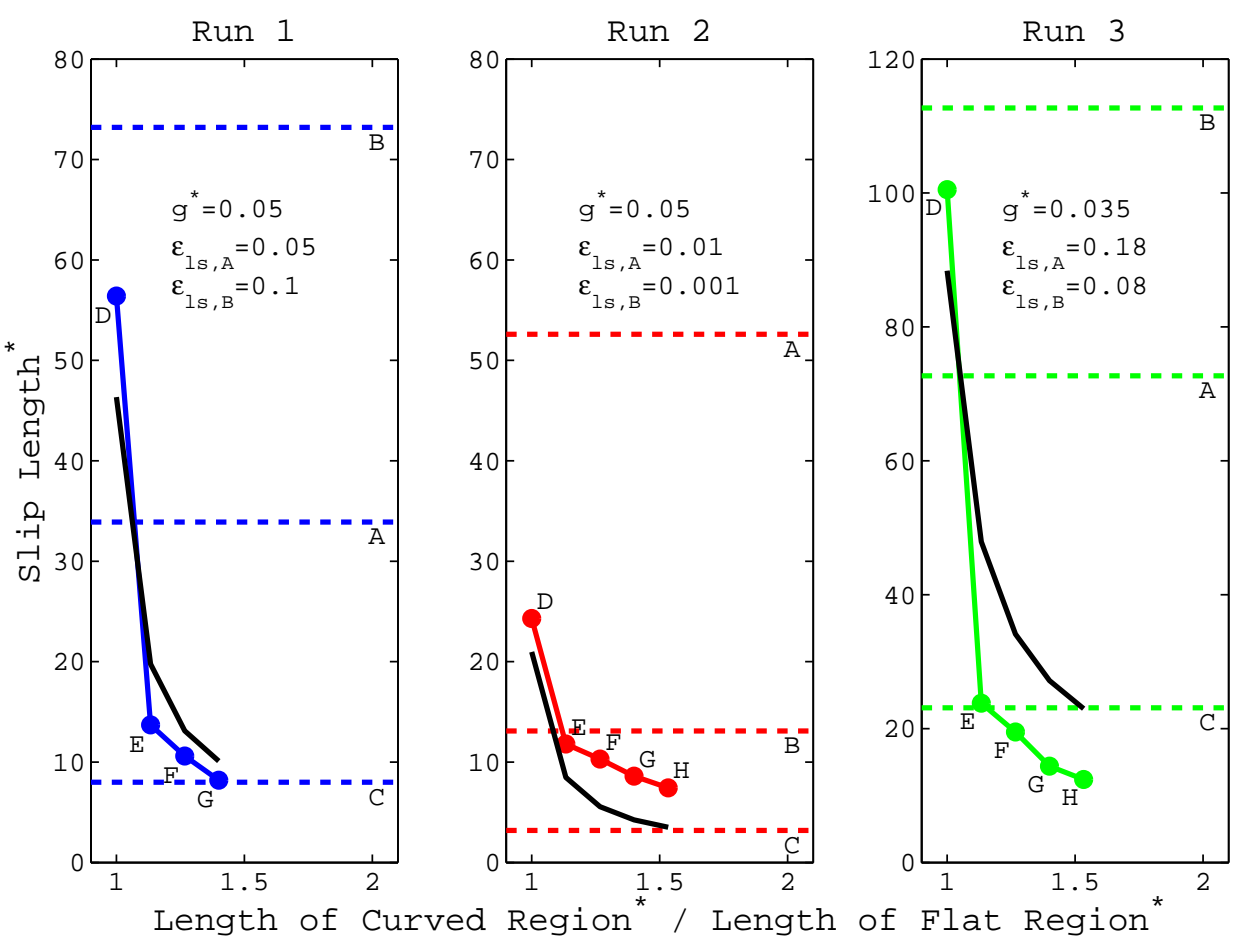

Figure 3.9: Slip lengths of surfaces A-H. Slip lengths are plotted as against the ratio of the length of the curved region to the flat region. Dashed lines are the slip lengths of surfaces A-C and circles are D-H. Run 1: $g^{*}=0.05, \epsilon_{l s, A}=0.05, \epsilon_{l s, B}=0.1$. Run 2: $g^{*}=0.05$, $\epsilon_{l s, A}=0.01, \epsilon_{l s, B}=0.001$. Run 3: $g^{*}=0.035, \epsilon_{l s, A}=0.18, \epsilon_{l s, B}=0.08$. Run 1 (blue) does not have an estimate for surface $\mathrm{H}$ as the liquid in that simulation froze. The theory (Eq. 3.10) is plotted (black line) to compare with the data.

0.035. The $\epsilon_{l s}$ values of the simulations, from left to right, for the green surfaces were $0.5,0.01,0.18$ and for the cyan surfaces, $0.1,0.001,0.08$. Our choices for $\epsilon_{l s}$ may appear arbitrary. The reason for this was that creating large slip lengths for our viscous liquid was not trivial, and we had to experiment with different values of $\epsilon_{l s}$. The fact that a smaller $\epsilon_{l s}$ does not necessarily imply a larger slip was part of the challenge in finding good values of $\epsilon_{l s}$. The other challenge was that the energy parameter also affected the average distance between the liquid and the surface, and this affected our ability to properly estimate slip for surface C. Nonetheless, our chosen values provided satisfactory results with which to test the theory.

We also plot the theory in Figure 3.9 for comparison. Note that our surface is actually a tertiary surface, so Eq. 3.10 becomes,

$$
b_{\mathrm{eff}, i}=\left[\frac{\beta_{\mathrm{A}, i} s_{\mathrm{A}}}{b_{\mathrm{A}}}+\frac{\beta_{\mathrm{B}, i} s_{\mathrm{B}}}{b_{\mathrm{B}}}+\frac{\beta_{\mathrm{C}, i} s_{\mathrm{C}, i}}{b_{\mathrm{C}}}\right]^{-1},
$$

where the indices $\mathrm{A}, \mathrm{B}$, and $\mathrm{C}$ represent their surfaces, and the index $i$ is for the 


\begin{tabular}{|c|c|c|c|c|c|}
\hline & $\mathrm{D}$ & $\mathrm{E}$ & $\mathrm{F}$ & $\mathrm{G}$ & $\mathrm{H}$ \\
\hline$s_{\mathrm{A}, i}$ & 1 & 1 & 1 & 1 & 1 \\
$s_{\mathrm{B}, i}$ & 1 & 1 & 1 & 1 & 1 \\
$s_{\mathrm{C}, i}$ & 0 & 2 & 2 & 2 & 2 \\
$\beta_{\mathrm{A}, i}$ & 0.50 & 0.47 & 0.44 & 0.42 & 0.39 \\
$\beta_{\mathrm{B}, i}$ & 0.50 & 0.41 & 0.32 & 0.25 & 0.18 \\
$\beta_{\mathrm{C}, i}$ & 0 & 0.13 & 0.24 & 0.33 & 0.42 \\
\hline
\end{tabular}

Table 3.2: Parameters for calculating effective slip. Normalized arc lengths, $s$, and area fractions, $\beta$, for surfaces D-H. The $i$ index takes surfaces D-H. For example, $s_{\mathrm{C}, \mathrm{D}}$ is the normalized arc length of the $\mathrm{C}$ surfaces within the $\mathrm{D}$ surface.

mixed surfaces D-H. The normalized arc lengths for surfaces A and B are always 1 because the arc length and interval for these two surfaces are always the same (the interval length is length of the arc in the x-direction, see Figure 3.7 ). $s_{\mathrm{C}, i}$ is always 2 (except for $i=\mathrm{D}$ ) since each cut in the lattice results in an arc length of 2 atoms ( 1 atom in both $\mathrm{x}$ and $\mathrm{y}$, where an additional $\mathrm{C}$ surface atom is taken from a B surface atom) while the interval is 1 atom in length. The normalized arc lengths as well as the area fractions of each surface are presented in Table 3.2. The data in the table and the estimated slip lengths for surfaces A-C are used to generate the curves for $b_{\text {eff }}$ in Figure 3.9.

\subsubsection{Discussion}

The most prominent feature of the results in Figure 3.9 is the large drop in slip length from surface D to E. Surface D is only made up of surfaces A and B, which always have higher slip lengths than C. Surfaces E-H are mixes of surfaces A-C, so the low slip length of $\mathrm{C}$ greatly affects the effective slip length. According to the model proposed by Nat Lund et al. [20], the effective slip length of a system made up of smaller inherent slip lengths is dominated by the smallest inherent slip. In our case, the (111) surface has the lowest slip length and it causes the effective slips of surfaces E-H to be much smaller. This is evident in the computed effective slip lengths from Eq. 3.11 plotted in Figure 3.9. For Run 1 we see very good agreement of the theory with our results. The theory captures the proper behavior of the data in Runs 2 and 3, but the model appears to underestimate slip in Run 2 and overestimate slip in Run 3.

In Run 3, we see that surfaces E-H are estimated to have smaller slip lengths than C. This may be caused by an improper estimate of the slip length of C. In Figure 3.10 we compare the snap shots of simulations of surface C from Runs 1 , 2, and 3. In Run 2 (middle), liquid atoms are much closer to the surface than 


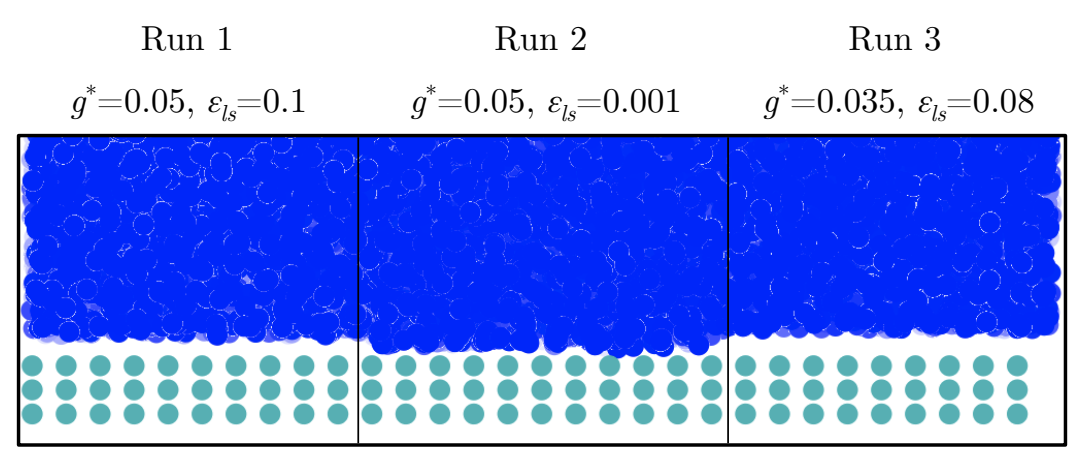

Figure 3.10: Comparing the $\mathbf{C}$ surfaces. Snapshots of simulations of surface $\mathrm{C}$ near the liquid-surface region are provided for Run 1 (left), Run 2 (center), and Run 3 (right). The liquid in Run 2 is much closer to the surface as a result of the small $\epsilon_{l s}$. The similarities between Run 1 and Run 3 cannot elucidate the cause for the high slip seen for surface C in Run 3.

in the other runs. This is due to the small $\epsilon_{l s}$ which lowers the strength of the repulsive potential of the surface. Runs 1 and 3 appear very similar except for the slight increase in distance between liquid and surface in Run 3, which in this case is caused by a lower gravity component. Perhaps this separation distance changes when mixing surfaces, and this prevents us from recording the proper slip.

In $\mathrm{MD}$, ordering of the liquid near the surface is an indication of wetting. Figure 3.11 shows the density profiles of the liquid for simulations of surface C. Oscillations of the density near the surface (height $\approx 0$ ) is an indication of ordering, so the liquid is partially wetting surface $\mathrm{C}$ for all runs. It is worth noting the change in density along the height profile. This is not the best case scenario for estimating the slip of the surface as the liquid is not uniform in density along the height. This gradient in the liquid density is a result of ordering caused by the surface, where the density tends towards the liquid density away from the surface (large height) and tends towards the surface density near the surface (small height). The important part is that these density profiles are consistent for the simulations of all surfaces for each run.

Figure 3.11 also shows that a higher gravity term (Runs 1 and 2) causes the density of the liquid to be larger near the surface, which indicates more ordering at higher pressures. Also, the choice of $g$ will cause the density to slightly increase if $g$ is larger than the force fluctuations within the liquid, and this is the case in our simulations as we see the density at the top of the liquid is slightly larger than 0.85 . There is little difference in the fluctuations near the surface between all runs. Run 2 has a slightly larger fluctuation which is expected since its separation distance is much smaller.

Perhaps the (111) surface alone is not a good representation of the steps in sur- 


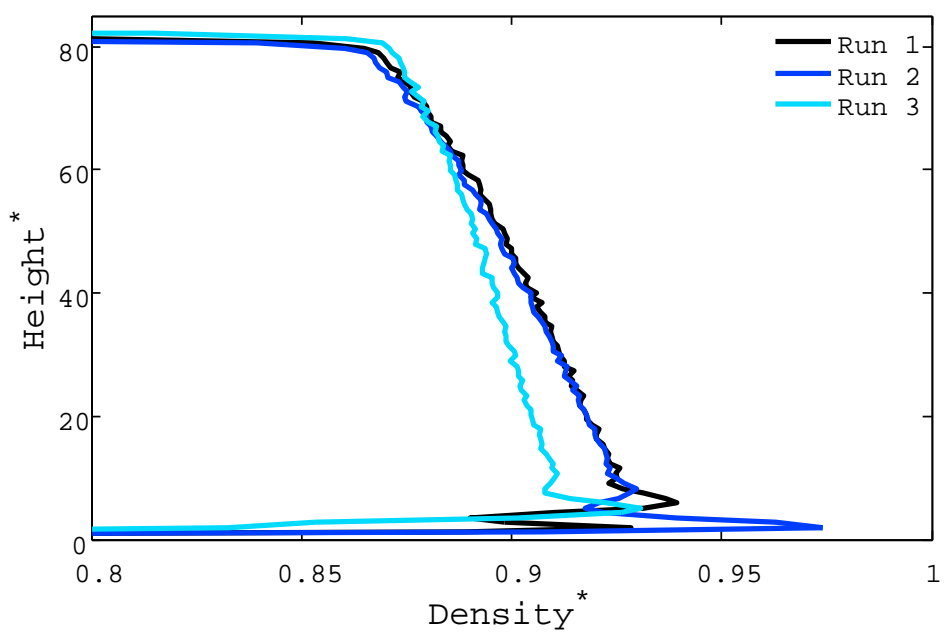

Figure 3.11: Density profiles of surface C. These density profiles confirm that the liquid partially wets surface $\mathrm{C}$ in all runs. The lower density in Run 3 is a result of a lower gravity. Perhaps the smaller ordering in Run 3 is a cause for the high slip of surface C.

faces $\mathrm{E}-\mathrm{H}$, especially considering the fact that surface $\mathrm{C}$ is flat. The change from the (010) to the (111) may represent a surface with a slightly larger roughness, thus resulting in a smaller slip length.

\subsubsection{Conclusion}

In this section, we demonstrated how to estimate the slip lengths of surfaces using MD. This was a simple task, and it has been done a number of times in the literature; however, we used MD to conduct a simulation to test the validity of a theory. Though estimating slip lengths is trivial, we were surprised with the results that we found, mainly the fact that simple cuts in an atomistic surface would greatly decrease the slip length. This makes sense with regard to surface roughness and even the theory; but, we did not expect to see these effects at such a small scale. These results reiterate the fact that fluid dynamics and the models developed are very scalable even down to the atomistic level. 


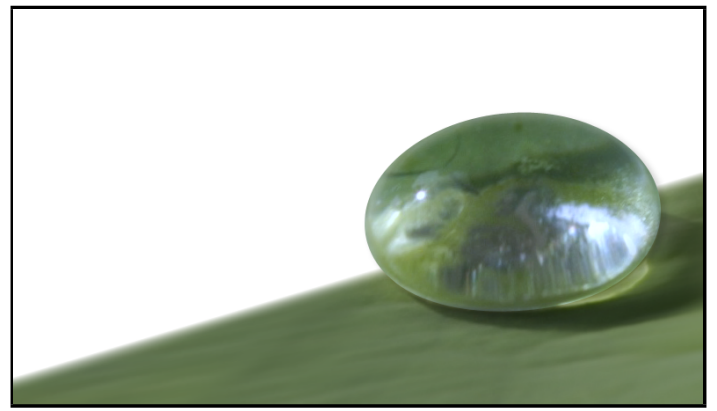

Figure 3.12: A liquid drop on an inclined, super-hydrophobic surface.

\subsection{Drop Dynamics on Slippery Surfaces}

The motion of liquid drops moving down a super-hydrophobic surface is well studied, and general scaling laws are shown to match well with experimental results $[22,23]$. Thus far theory and experiments have verified that drops can roll or slide down an incline, but few consider the case of drops rolling and sliding down an incline.

Observing and measuring rolling and slipping motion in drop experiments is difficult since these drops tend to be on the scale of millimeters. Previous work used pigmentation and high speed cameras in order to record rolling motion of viscous, liquid drops; however, the resolution of these methods makes it difficult to observe slip if it were present. Furthermore, experiments and theory have measured and predicted lengths of up to tens of microns (in some instances, hundreds of microns) [21] for fluids moving along a surface. This could imply that drops would need to be on the order of microns in size if slip is to play an important role in the drop dynamics.

One paper used MD to study sliding drops [24], and they found drop speed to increase with increasing size and slip length. Their surfaces were not superhydrophobic and they found the drop dynamics to be dominated by shearing due to slip. Another paper conducted simulations of drops moving along superhydrophobic surfaces [25], and they include details on the velocity profiles within the drop; however, they did not study the effects of drop size and slip length on drop dynamics.

Recently, Mark Hunter et al. [26] have proposed a method to measure velocity gradients inside of a moving drop of water by using nuclear magnetic resonance imaging techniques. They hope to conduct experiments on super-hydrophobic surfaces, and this research will hopefully produce experimental results on slipping and rolling of liquid drops.

In this section, we will use MD to study the motion of liquid drops on super- 


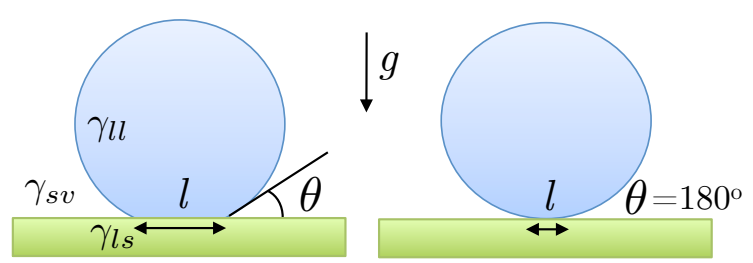

Figure 3.13: A drop on a hydrophobic surface. The angle of contact between a liquid drop and a surface is $\theta$, and this angle arises from a balance in surface tension between the liquid, surface, and vapor. For $\theta<180^{\circ}$ the drop will wet the surface (left) with a contact size of radius $l$. For $\theta=180^{\circ}$, the drop is in a non-wetting state, but gravity, $g$, still causes the drop to have a finite contact size $l$ with the surface (right).

hydrophobic surfaces. We will study the effect of slip length and drop size on drop speed, and we will attempt to develop a simple theory for drop speed that takes into account these parameters. The surface we will use to create superhydrophobicity will have a geometry similar to Figure 3.5

\subsubsection{Theory}

Here, we will develop a scaling law that aims to predict the behavior of a drop moving down an inclined, super-hydrophobic surface. Scaling laws tell us, for example, how drop speed is affected by drop size, and these types of laws are typically valid at certain extrema when a particular function dominates. We will use the " $"$ to mean "scales as" in the theories to follow. We will start with theory developed thus far, and then move to develop our own theory.

\section{Static drops}

A drop of liquid resting on a surface makes a contact angle $\theta$ with a surface given by,

$$
\cos \theta=\left(\gamma_{s v}-\gamma_{l s}\right) / \gamma_{l l}
$$

where $\gamma$ is the surface tension between the solid-vapor, liquid-solid, and liquidliquid interfaces (see left diagram in Figure 3.13). Drops are said to wet a surface if they make a contact angle less than $90^{\circ}$ and partially wet a surface for angles between $90^{\circ}$ and $180^{\circ}$. Non-wetting is the case where $\theta=180^{\circ}$. A surface is hydrophobic for angles greater than $90^{\circ}$ and super-hydrophobic for angles greater than $160^{\circ}$. The shape of a drop will be nearly spherical if the drop radius is smaller than the capillary length [23],

$$
\kappa^{-1}=\sqrt{\frac{\gamma}{\rho g}}
$$


where $\rho$ is the density of the liquid and $g$ is gravity. For the case of drops smaller than $\kappa^{-1}$, the contact size that the drop makes with a surface can be approximated as a function of the contact angle and the drop radius $R$ [23],

$$
l \sim 4^{1 / 3} \frac{\sin \theta}{(2+\cos \theta)^{1 / 3}(1-\cos \theta)^{2 / 3}} R .
$$

In the limit as $\theta \rightarrow \pi$, the contact size tends to zero; however, gravity will cause the drop shape to deform slightly, thus giving rise to a finite contact size. For non-wetting drops smaller than the capillary length [23],

$$
l \sim R^{2} / \kappa^{-1}
$$

This relation arises from a balance between gravity and surface tension. The right diagram in Figure 3.13 provides an example of a non-wetting drop with a small contact size $l$.

\section{Dynamic drops}

When a spherical drop is initially placed on a surface, its instantaneous contact angle will be $180^{\circ}$. Then, depending on the liquid-solid interactions, the contact angle will relax to an equilibrium as given by Eq. 3.12. The dynamics of this contact angle relaxation determines how quickly a liquid will wet a substrate, and molecular kinetic theory explains these macroscopic dynamics using microscopic techniques. The contact line of a liquid with a solid is motivated by the out-ofbalance surface tension forces. By considering the frequency at which molecules in a liquid hop between atom sites on a surface, Blake showed that the speed of the contact line as a function of contact angle is given by [27],

$$
v=2 K^{0} \lambda \sinh \left[\frac{\gamma_{l l}\left(\cos \theta^{0}-\cos \theta\right)}{2 n k_{B} T}\right] \text {, }
$$

where $K^{0}$ is the frequency at which the contact line jumps, $\lambda$ is a molecular hopping distance, $n$ is the number of absorption sites per unit area, $\theta^{0}$ is the equilibrium contact angle, and $k_{B} T$ is the Boltzmann constant times temperature. The evolution of the contact line can also be calculated by considering the contact size, $l$. For small drops, $R \ll \kappa^{-1}[28]$,

$$
\frac{\partial l}{\partial t}=-\frac{\partial \theta}{\partial t} \frac{(1-\cos \theta)^{2}}{\left(2-3 \cos \theta+\cos ^{3} \theta\right)^{4 / 3}} .
$$


Using Equations 3.16 and 3.17, one can compute $K^{0} \lambda$ and $n$ by finding the evolution of $\theta$. By relating the contact angle velocity to a driving force proportional to $\gamma_{l l}\left(\cos \theta^{0}-\cos \theta\right)$, one can define a coefficient of friction as [29],

$$
\zeta=\frac{\eta m}{\rho \lambda^{3}}
$$

where $\eta, m$, and $\rho$ are the liquid viscosity, mass, and density, respectively, and we assume an equilibrium contact angle of $180^{\circ}$.

\section{Drops moving down an incline}

We want to find the steady-state speed of drops moving down an incline. In order to do this, we will need to make a few assumptions. First, we must have a liquid drop sufficiently viscous such that its spherical shape is not deformed by its speed and such that viscous effects dominate inertia. Thus we require a capillary and Reynolds number much less than unity, $C a=\eta U / \gamma \ll 1$ and $R e=\rho l U / \eta \ll 1$ [30]. From the properties of our L-J liquid (Table 3.1) ${ }^{6}$, we require speeds $U^{*} \ll 0.46$ for small $C a$. For small $R e$, we require $U^{*} \ll 4 / l$. We will only consider angles of inclination $\alpha \ll 1$ so that the force motivating drop speed is low, allowing us to maintain our low speed requirements.

A drop moving down an incline will reach a terminal speed related to a balance between the change in potential energy and dissipation,

$$
\rho R^{3} g U_{c m} \alpha \sim D
$$

where density, gravity, drop radius, speed, and dissipation are $\rho, g, R, U_{c m}$, and $D$ respectively, and $U_{c m} \alpha$ represents the rate of change of the gravitational potential. Here we define the speed of the center of mass as the drop speed, and we also take the approximation $\sin \alpha \approx \alpha$.

\section{Purely rolling drops}

Consider Figure 3.14. Assume there is a small volume in the drop where energy is lost to viscosity (dark blue region), and assume that the velocity gradient throughout the drop is similar to that of a rigid body rolling down an incline (right diagram, shown as a superposition of rotation and translation). Viscous dissipation in the drop will be confined to the contact region, and the volume of this region is $V_{d} \sim l^{3}$. The velocity gradient in the contact region is $|\nabla \mathbf{u}| \sim$

\footnotetext{
${ }^{6}$ Both $C a$ and $R e$ are dimensionless quantities, so $R e^{*}=R e$ and $C a^{*}=C a$
} 

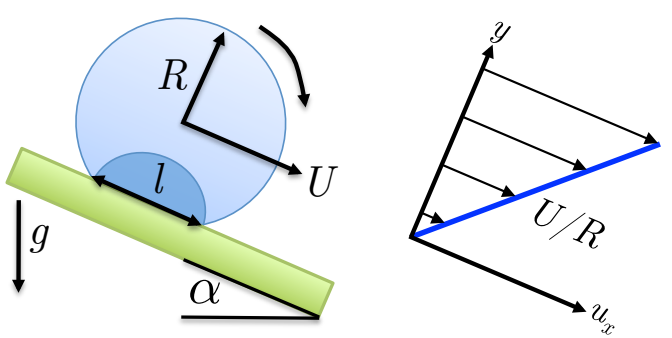

Figure 3.14: Diagram of a rolling drop. A drop of radius $R$ rolling down an incline of angle $\alpha$ (left) is motivated by gravity $g$, and viscous dissipation (shaded region) acting on a volume proportional to the contact size, $l$, causes the drop to reach a terminal velocity of $U$. The velocity profile of the drop is shown (right) and has a gradient of $U / R$. Note that translation is included in this velocity profile, hence the zero velocity at $y=0$.

$U_{c m} / R$, and the viscous dissipation is defined as,

$$
D \sim \eta \int_{V}|\nabla \mathbf{u}|^{2} d V
$$

Solving Equation 3.19 together with Equations 3.13, 3.20, and 3.15, we find that these drops reach a terminal speed,

$$
U_{c m} \sim \frac{\gamma}{\eta} \alpha \frac{\kappa^{-1}}{R}
$$

indicating that rolling drops increase in speed as their size decreases. This scaling law was shown to match experiments quite well even for drops where $R=\kappa^{-1}$ [30]. Note that since there is only rolling motion, the rolling speed $U_{r}=U_{c m}$.

For puddles, drops larger than $\kappa^{-1}$, the drop will flatten out and the height of the drop will tend to $2 \kappa^{-1}$ [23]. This means that we can assume the radius, height, and contact size of the drop scale as $\kappa^{-1}$. The viscous forces in these puddles will be $\eta \kappa^{-2} U_{c m} / \kappa^{-1}$, where friction acts on a contact area $\kappa^{-2}$ and $U_{c m} / \kappa^{-1}$ is the shear rate in the drop. The gravitational force is $\rho \kappa^{-3} g \alpha$, and the balance between viscous and gravitational forces predict a terminal speed of [23],

$$
U_{c m} \sim \frac{\gamma}{\eta} \alpha
$$

which is consistent with Eq. 3.21 for $R=\kappa^{-1}$. 

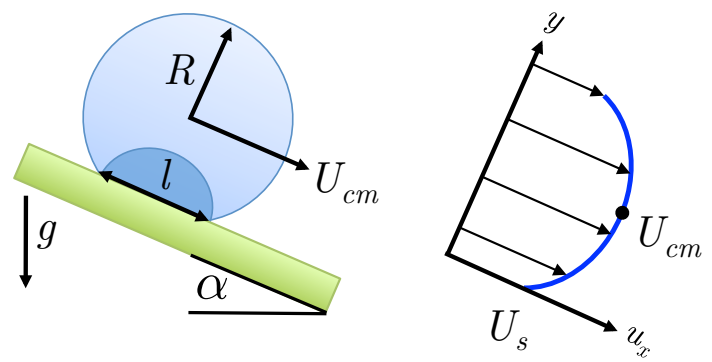

Figure 3.15: Diagram of a sliding drop. A drop of radius $R$ sliding down an incline of angle $\alpha$ (left) is motivated by gravity $g$, and the velocity profile of the drop (right) results from the Navier-Stokes equation of a liquid motivated by a force. The drop slips with speed $U_{s}$, and the drop's speed, $U_{c m}$, is the flow at the height of the center of mass of the drop.

\section{Purely sliding drops}

For the case of a drop sliding down an incline, we can use the Navier-Stokes equation for a fluid flow motivated by a force [24],

$$
\frac{\partial^{2} u_{x}}{\partial y^{2}}=-\frac{\rho g \alpha}{\eta}
$$

where $g \alpha$ is the component of gravity parallel to the surface. Figure 3.15 provides a diagram of a drop sliding down an incline. $U_{s}$ is the slip speed at the liquid-solid interface, and the drop speed is $U_{c m}$ at the center of mass of the drop. In the case of slip, there is a non-zero shear rate at the liquid-surface boundary (the dark blue region). The Navier slip boundary condition (Eq. 3.9) along with Eq. 3.23 results in,

$$
u_{x}(y) \sim-\frac{\gamma}{\eta} \frac{y^{2}}{\kappa^{-2}}+\frac{U_{s}}{b} y+U_{s} .
$$

We can estimate the slip speed by balancing the force of gravity with the frictional forces arising from slip. There are two types of friction that we predict will be important at the contact zone. First is the force of friction due to shear, $U_{s} / b$, acting on an area $l^{2}, f_{A} \sim \eta l^{2} U_{s} / b$. Second is a frictional force acting on the perimeter of the contact zone, $f_{C} \sim \zeta l U_{s}$, where $\zeta$ is a coefficient of friction. Balancing these forces with gravity, we find,

$$
U_{s} \sim \frac{\gamma \alpha R b}{\eta R^{2}+\zeta \kappa^{-1} b}
$$

For the case of small drops and large slip, $R \ll \kappa^{-1}$ and $R \ll b$, we expect $U_{c m} \sim U_{s}$. 


\section{Rolling and slipping drops}

At the heart of the models for purely rolling or sliding drops, as well as other models that attempt to predict drop speed [24], is the idea of balancing energy dissipation; that is, if a moving drop is to reach a steady-state, then the total energy of the system must remain constant. The observation that drops do indeed reach a steady-state was made in experiments [31,32].

We define the total energy dissipation in a drop moving down an incline as,

$$
D_{t}=D_{v}+D_{a}+D_{c}
$$

where $D_{v}$ is viscous dissipation due to rolling, $D_{a}$ is the dynamic friction caused by slip at the liquid-surface interface, and $D_{c}$ is dissipation due to contact line dynamics. We have shown what these dissipation terms look like, and we will summarize them here. A drop will begin to roll because viscous dissipation within the contact zone will induce a torque on the drop. This dissipation is,

$$
D_{v} \sim \int_{V} \eta\left(\frac{U_{r}}{R}\right)^{2} d V \sim \eta l^{3}\left(\frac{U_{r}}{R}\right)^{2}
$$

where we take the volume integral in the contact region. The force of dynamic friction aries from shear at the liquid surface interface acting on the contact zone, which has an area $\sim l^{2}$. The dissipation at the surface of the contact zone is,

$$
D_{a} \sim U_{s} \int_{A} \eta \frac{U_{s}}{b} d A \sim \eta l^{2} \frac{U_{s}^{2}}{b},
$$

where we assume the rate of dissipation is proportional to $U_{s}$, and we take the area integral at the liquid-surface contact zone. Contact line dynamics predicts a friction force, $F=\zeta u$. Assuming this friction acts along the perimeter of the contact zone and that the rate of dissipation is proportional to $U_{s}$, we find,

$$
D_{c} \sim U_{s} \int_{C} \zeta U_{s} d C \sim \zeta l U_{s}^{2},
$$

where the integral is taken along the contact line.

We expect the total dissipation of the drop to be balanced by the change in gravitational potential energy. From this energy balance, along with the above 
equations, we find the relation,

$$
U_{0} U_{c m} \sim \frac{R}{\kappa^{-1}} U_{r}^{2}+\frac{R}{b} U_{s}^{2}+\frac{\zeta}{\eta} \frac{\kappa^{-1}}{R} U_{s}^{2}
$$

where we have used the puddle speed $U_{0}=\gamma \alpha / \eta$ and we assume the relation 3.15 , which requires non-wetting drops where $R<\kappa^{-1}$. Note that all parameters are accounted for except $\zeta$, which we will cover in the results below. Finally, we expect $U_{c m} \sim U_{s}+U_{r}$. Since we are interested in steady-state solutions, we can take the derivative of Eq. 3.30 with respect to $U_{r}$ and $U_{s}$, noting that $\partial U_{s} / \partial U_{r}=\partial U_{r} / \partial U_{s}=0$. From this, we could solve Eq. 3.30; however, we will keep our scaling law as dependent on $U_{r}$ and $U_{s}$. This makes the law more convenient for comparison with our results. Alternatively, we could assume rolling dynamics and slipping dynamics are independent of each other, in which case $U_{c m}$ would be the sum of Equations 3.21 and 3.25. We will test both of these possibilities with the results below.

\subsubsection{Simulation}

Snap shots of the simulation set up are provided in Figure 3.16. We simulated an incline by varying forces parallel and perpendicular $\left(f_{x}\right.$ and $\left.f_{y}\right)$ to the surface. For small inclinations, $\alpha \sim f_{x} / f_{y}$, and $f_{y}$ is the gravity term $g$. We vary the interaction potential between the liquid and the surface $\left(\epsilon_{l s}=0.15,0.2\right)$ in order to vary the slip length of the surface, and we also used rough surfaces in order to achieve large contact angles and super-hydrophobicity. The rough surface consists of equally spaced posts in three dimensions (Figure 3.16). Slip lengths were calculated as in Section 3.3, and they were also estimated directly from our drop simulations. These two estimates were relatively consistent.

Drop temperature was thermostatted by scaling atom velocities while ignoring the atom velocities in the plane of rotation (this meant that drop velocities were scaled in the $z$-direction only). The temperature was $T^{*}=1 \pm .02$ and it was checked every 100 units of time. Proper thermostatting is important in our simulations as we expect the drop to lose energy through dissipation. Since we expect the drop's motion to be primarily within the $x y$-plane, we can safely thermostat in the $z$-plane without affecting drop dynamics. The main requirement for our thermostat is that our surface has a temperature of zero because it is fixed. Fixing the surface while thermostatting the liquid is more computationally efficient.

Simulations took about $5 \times 10^{6}$ time steps for drops to reach steady state, and 


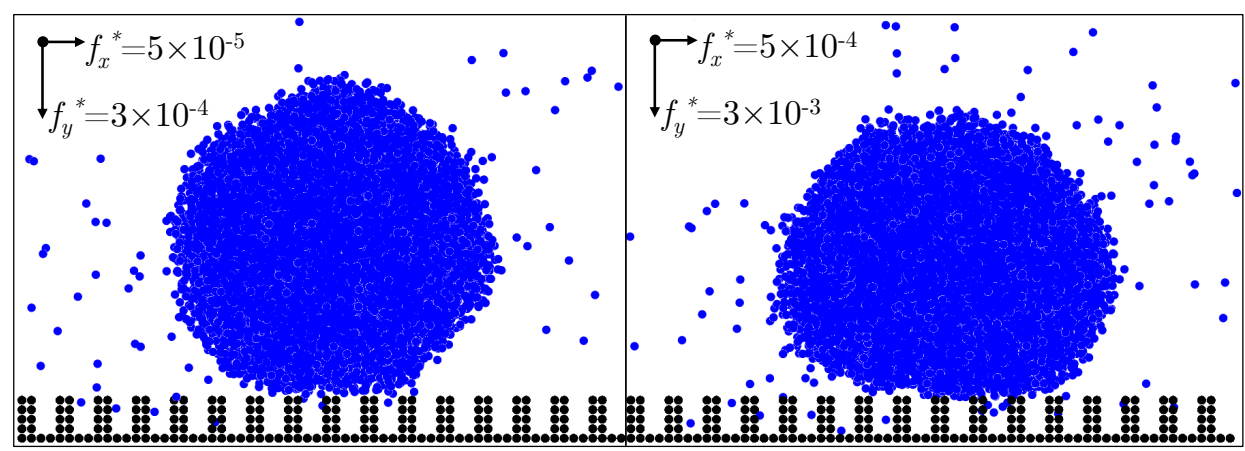

Figure 3.16: Snap shots of liquid drops. A snap shot of a liquid drop on a rough, superhydrophobic surface for $g^{*}=3 \times 10^{-4}$ (left) and $g^{*}=3 \times 10^{-3}$ (right). The surface consists of evenly spaced posts in three dimensions, and the surface is periodic in $x$ and $z$ while the simulation had a fixed boundary condition in $y$. The posts create a super-hydrophobic surface as evidenced by the high contact angle between the liquid (blue) and the surface (black). The drops above have radii of $R^{*}=13$.

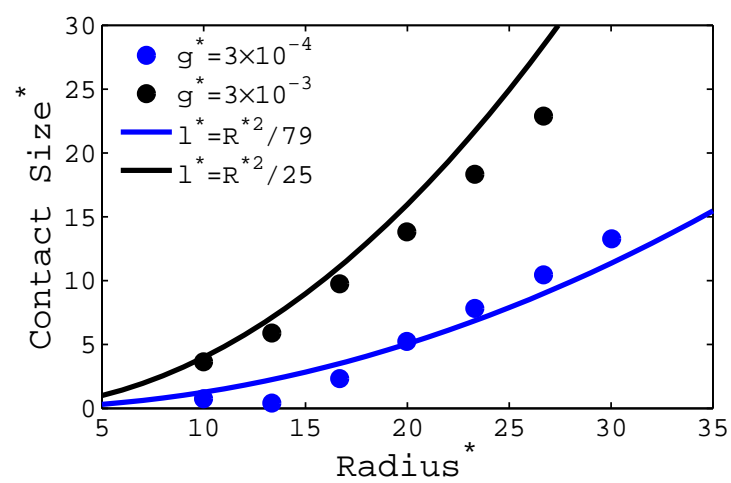

Figure 3.17: Contact size. Estimated drop contact sizes $(\bullet)$ are plotted against drop size. The theory for non-wetting drops says that the contact size should increase as $R^{2}$, and the theory matches our data reasonably well.

data were collected and averaged over another $25 \times 10^{6}$ time steps. Every $10^{3}$ time steps represents 1 unit of time, $\tau^{*}$.

\subsubsection{Results and Analysis}

\section{Contact Size}

Details on how we estimated a drop's contact size are provided in Appendix C. We present our estimated contact sizes along with the theory (Eq. 3.15) in Figure 3.17. We conducted simulations with two different gravities, $g^{*}$, of $3 \times 10^{-4}$ and $3 \times 10^{-3}$, resulting in two capillary lengths of 79 and 25 . Our estimates for the contact size, $l$, are in good agreement with the theory for the data with low $g$ (blue) and high $g$ (black). Since Eq. 3.15 is valid for drops smaller than $\kappa^{-1}$, we see better agreement at smaller radii for the high gravity case. 


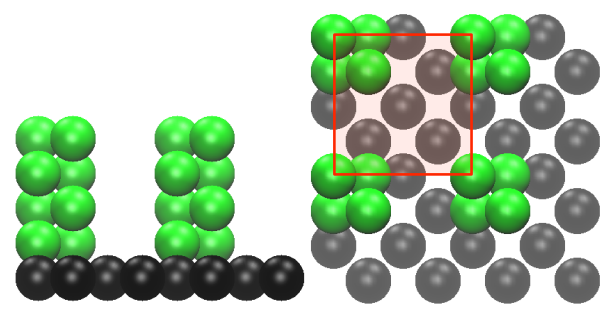

Figure 3.18: Posts of a super-hydrophobic surface. Here we show the surface used in our simulations. The unit cell (red box) is a square of length 3.17, and each cell contains one post (green). Each post is made of 8 atoms, and we calculated a molecular hopping distance of $\lambda^{*} \approx 1.25$ for our surface.

\section{Contact Line Friction}

We need the hopping distance, $\lambda$, and the number of absorption sites per unit area, $n$, in order to estimate $\zeta$ from Eq. 3.18. For evenly distributed surfaces, $\lambda=1 / \sqrt{n}$. Figure 3.18 provides a close look at the posts of the surface used in our simulations, and we see that we have at the least 2 absorption sites per unit cell, where the unit cell is a square of length 3.17. This value results in a molecular hopping distance of 2.24 , and we find $\zeta^{*}=0.37$.

We can improve upon this estimate by finding $n$ using the methods of Blake et al. $[28,33]$ and Equations 3.16 and 3.17. A drop was placed on surfaces with varying $\epsilon_{l s}$, and the contact angles were recorded over time. ${ }^{7}$ Figure 3.19 shows the time evolution of drop contact angles for different $\epsilon_{l s}$ parameters $(-)$, and a fit of the time evolution of $\theta$ is also plotted (--). The theory fits the data well especially for larger $\epsilon_{l s}$ where the data are less noisy and estimates of the contact size improve. ${ }^{8}$ Notice that at $\epsilon_{l s}=0.2$, the value used for one of our surfaces in the drop simulations, the contact angle is very close to $180^{\circ}$, an indication that there is little wetting and a requirement for our theory. Note that our results presented in Figure 3.19 are consistent with Figure 2 of Blake et al. [28]. We found $\zeta^{*}=2.1$ for our surface, indicating a molecular hopping distance of $\lambda^{*}=1.25$ and 0.64 absorption sites per unit area (roughly 6.5 atoms). With each post having 8 atoms, this value of $\zeta$ seems reasonable. Note that $\zeta$ is related to the geometry of the surface, so regardless of the chemical potential of a surface (or the $\epsilon_{l s}$ parameter we use in our simulations) $\zeta$ will be the same.

\footnotetext{
${ }^{7}$ Contact angles were difficult to estimate, so we used Equation 3.14 to determine $\theta$ from $l$.

${ }^{8}$ Noise in contact angle estimates increases for larger contact angles because our methods for estimating contact size do not work well as contact size approaches zero.
} 


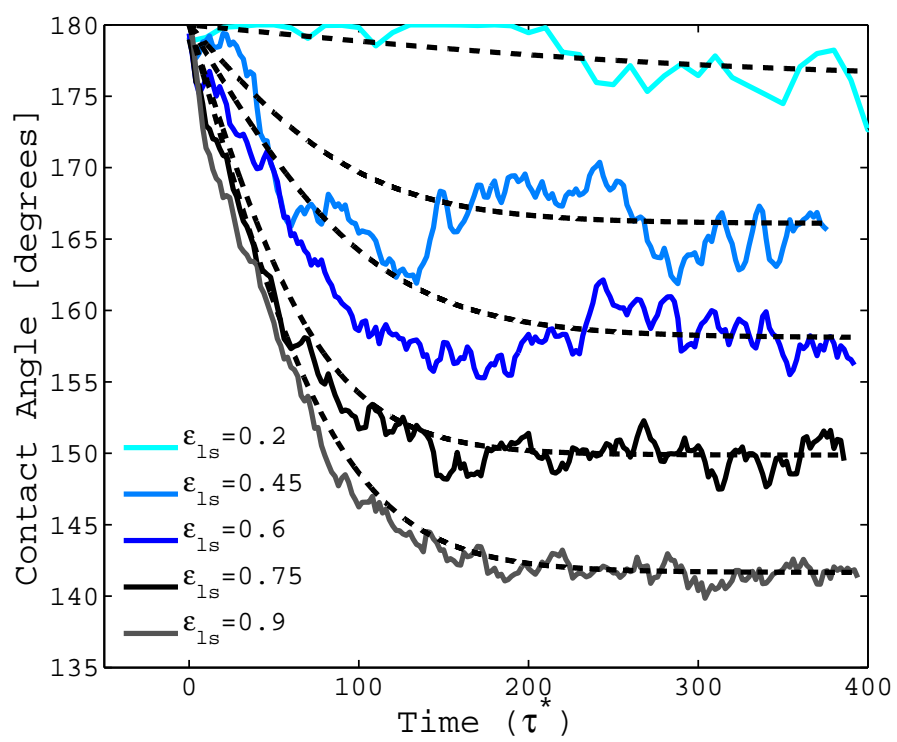

Figure 3.19: Contact angle. The time evolution of the contact angles for a drop of $R^{*}=16.7$ placed on the surface in Fig. 3.18 for different values of $\epsilon_{l s}$. The fits given by the dashed lines allow us to estimate the hopping distance, $\lambda$, and the number of absorptions sites per unit area, $n$. For clarity, some data points in the Figure were removed.

\section{Drop Velocity}

Parameters of the various simulation runs and results are presented in Figure 3.20. We plot the speed of the drop scaled by the angle of inclination versus the radius of the drop scaled by the capillary length. The speed of the drop, $U_{c m}$, is the time averaged speed of the center of mass of all atoms in the simulation. ${ }^{9}$ Since there are a small number of atoms in the vapor phase, this method provides a good estimate of the drop's total speed. Note that data were averaged after an initial transient state, which took around $5 \times 10^{6}$ time steps.

In Figure 3.20 we see a $1 / R$ trend similar to Eq. 3.21, the pure rolling model. We also see that $U_{c m}$ scales relatively proportionately with $\alpha$ as indicated by the similarities between simulations 1 and 3 and between simulations 2 and 4 . The data clearly show an increase in drop speed with increasing slip length. Simulations 5 and 6 appear to have a behavior much different from the other simulations - drop speeds are increasing with size. It may be difficult to compare these two sets of data with simulations 1 through 4 as the different capillary length indicates drops in a different regime.

\footnotetext{
${ }^{9}$ Though a force is applied in the $x$-direction only $\left(f_{x}\right)$, drops, especially small ones, tend to move in the $z$-plane. This effect may be attributed to the post structure of the surface (the post spacing is on the order of the drop size for small drops) as well as contact line dynamics. Though $U_{x} \gg U_{z}$, we record $U=\sqrt{U_{x}^{2}+U_{z}^{2}}$ as the velocity.
} 


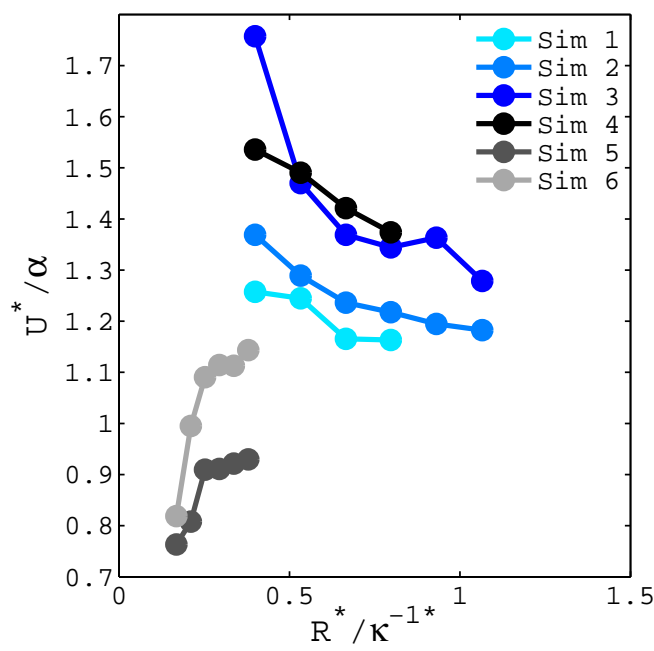

\begin{tabular}{|c|c|c|c|c|}
\hline Sim & $g^{*}$ & $\alpha$ & $\kappa^{-1 *}$ & $b^{*}$ \\
\hline \hline 1 & $3 \times 10^{-3}$ & 0.17 & 25 & 38 \\
2 & $3 \times 10^{-3}$ & 0.08 & 25 & 48 \\
3 & $3 \times 10^{-3}$ & 0.08 & 25 & 38 \\
4 & $3 \times 10^{-3}$ & 0.03 & 25 & 48 \\
5 & $3 \times 10^{-4}$ & 0.12 & 79 & 115 \\
6 & $3 \times 10^{-4}$ & 0.08 & 79 & 158 \\
\hline
\end{tabular}

Figure 3.20: Drop results. Drop speed scaled by the angle of inclination, $U^{*} / \alpha$, versus drop size scaled by the capillary length, $R^{*} / \kappa^{-1} . \alpha$ is in radians.

\section{Velocity Profiles}

Here we present some figures of the velocity profiles of the drops. These profiles will allow us to identify rolling and slipping motion. These profiles were recorded by slicing the simulation box along the $y$-direction and recording the averaged spacial velocities, orthogonal to $y$, in each slice. These spacial averages were then averaged over a large number of time steps. The result, $U(y)$, is the speed (parallel to the surface) inside the drop and along the height of the drop.

Our method of recording the velocity profiles makes sense for fluid flow, but it may not be clear that this method will give us the correct profile of a rolling, sliding drop. To prove that these spacial averages give us a proper estimate of the drop profile, consider two cases, a drop purely sliding in $x$ and a drop purely rolling in $x$ and $y$ with translation in $x$. In the case of pure slip, a drop's speed along its height is constant, $u_{x}=u_{s}$, so spacial averages of $u_{x}$ along $y$ will give us the correct profile. If a drop is purely rolling, the velocity components in radial coordinates are $u_{x}=r \omega \sin \theta+u_{t}$ and $u_{y}=-r \omega \cos \theta$, where $\omega$ is the angular velocity of the drop, $\theta$ is the angular position of a point in the drop, and $u_{t}$ is some translational speed. Converting $u_{x}$ to cartesian coordinates, we find $u_{x}=y \omega+u_{t}$, which implies that the velocity inside the drop and parallel to the surface is the same throughout the drop at height $y$. Whether a drop slides, rolls, or both, the spacial average of $u_{x}$ at some height $y^{\prime}$ is,

$$
u_{x, \operatorname{avg}}\left(y^{\prime}\right)=y^{\prime} \omega+u_{t},
$$



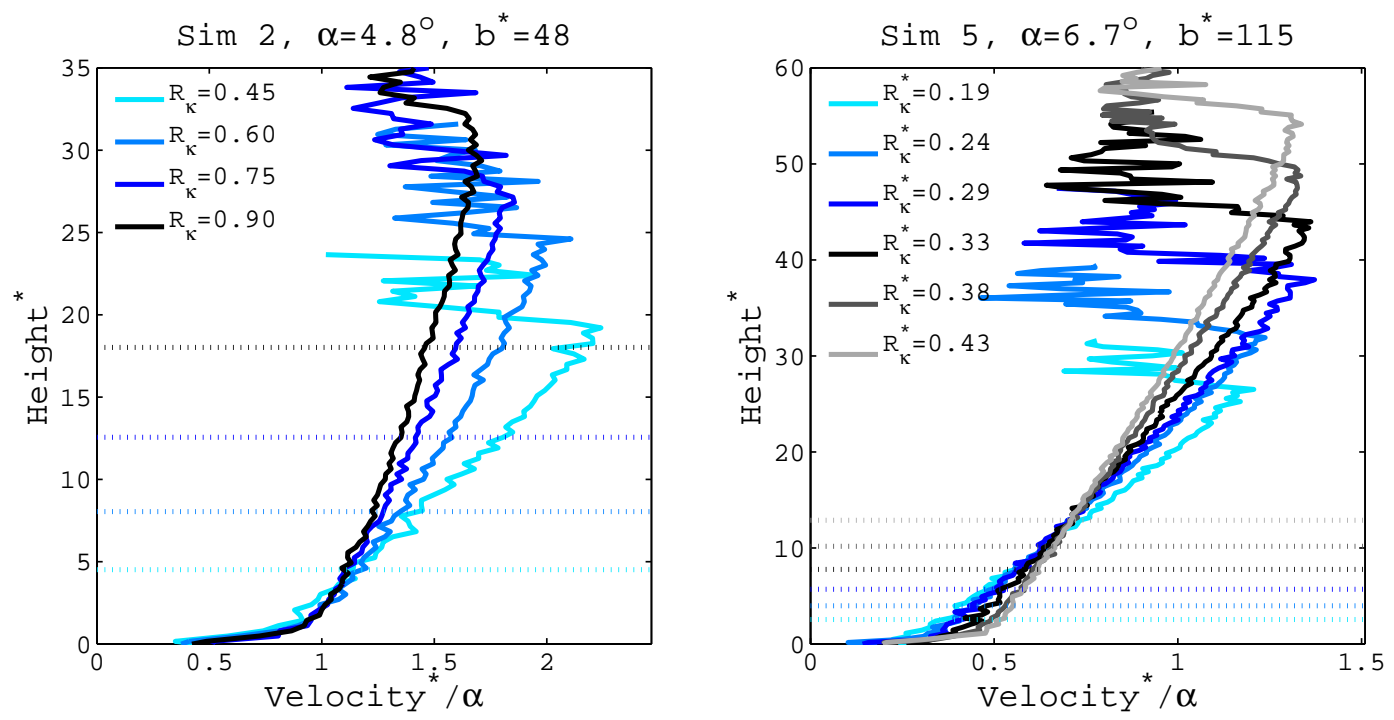

Figure 3.21: Velocity profiles versus size. The speed within the drop is plotted along the height of the drop for simulations 2 and 5 (left and right). $R_{\kappa}$ is the scaled radius, $R / \kappa^{-1}$, and the dotted line is an estimate of the height of the contact size, $l$, within the drop.

where $u_{t}$ may include slip in addition to center of mass translation. Note that Eq. 3.31 is consistent with the velocity profile of a solid sphere rolling and translating. This method of recording rolling drop profiles is also consistent with others [24].

Figure 3.21 contains velocity profiles from simulations 2 and 5, and the graphs demonstrate the effect of drop size on the velocity of the drop. The linear region in the velocity profiles are consistent with rolling motion, and the increase in slope indicates that rolling motion decreases as drop size increases. Prominent in simulation 5 (right) is an increase in slip speed as drop size increases. Slip speed is roughly the velocity of the drop near $y=0$. The estimated contact size is shown (dotted line) as an indication of the height of the viscous dissipation region within the drop. The noisy tails at the top of the drop profiles are atoms in the vapor phase, and they can be ignored.

Figure 3.22 compares the profiles of drops with different angles of inclination (left) and with different slip lengths (right). We see very little effect of $\alpha$ and slip length on the slope of the velocity profiles, indicating little effect on rolling speed; however, we do see an effect of these parameters on slip. When comparing velocities from drops with different $\alpha$, we would expect that the scaled velocities would be identical. On the contrary, we see a slight difference in the profiles from simulations 1 and 3. This may be error or noise in the data, and it is small enough that we may ignore it. When comparing the effect of slip length in simulations 2 and 3 , we see that a higher slip length shifts the velocity profiles. This indicates a change in $U_{s}$ while $U_{r}$ remains relatively constant. 

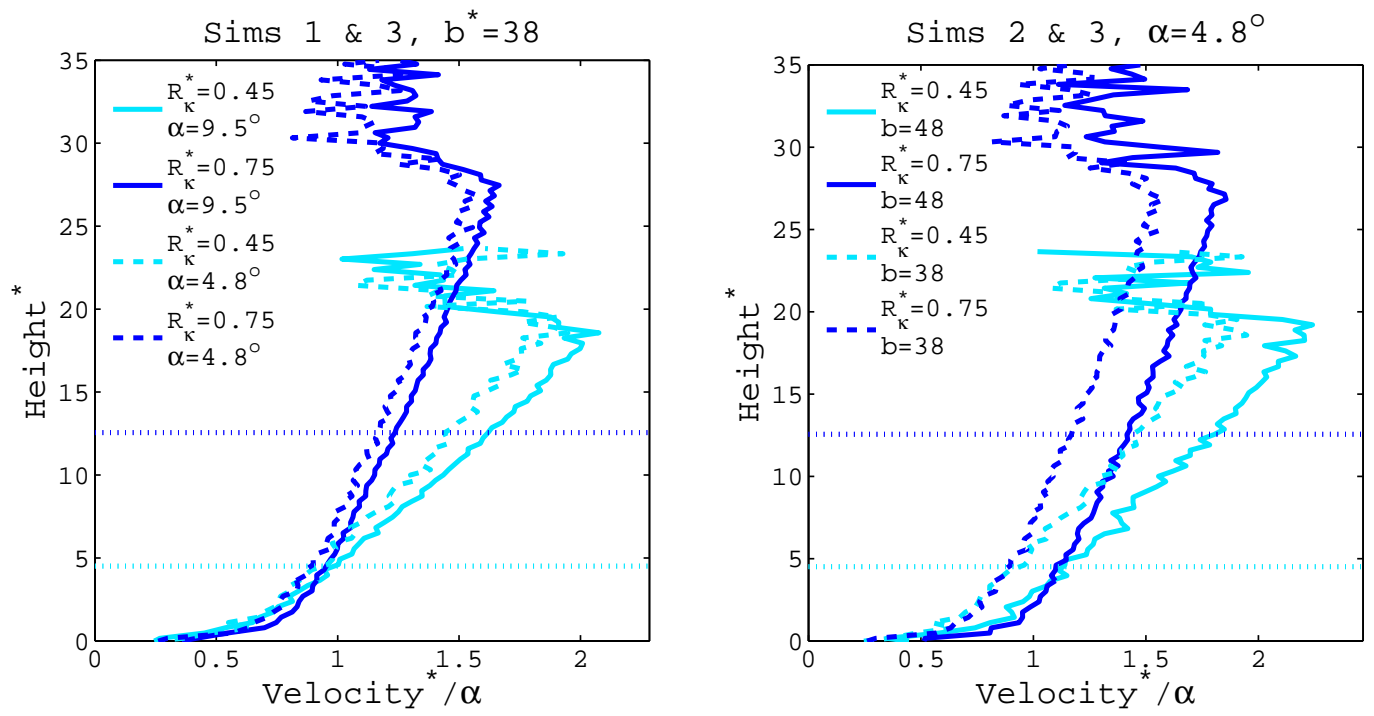

Figure 3.22: Comparing velocity profiles. In the left graph we plot drop profiles from simulations 1 and 3 which contain different angles of inclinations. Note that the velocity is scaled by $\alpha$, so we would expect to see similar profiles for each simulation. On the right we present profiles from simulations 2 and 3 , which contain surfaces of different slip lengths.

\section{Extracting Rolling and Slipping Speeds}

Velocity profiles like the ones in Figures 3.21 and 3.22 allow us to estimate rolling speed and slipping speed. It may not be clear at first what slipping speed ought to be - the criteria $U_{s}=U(0)$ doesn't necessarily apply - so we must create a model for the velocity profiles that is consistent with our results.

Considering Eq. 3.31 and the results indicating that our drops are both rolling and slipping, we can find the linear component of the velocity profile, $y \omega$, by fitting a line to the drop profiles. We can improve the accuracy of that fit by ignoring data in the contact zone, $y<l$, and by estimating the drop height. It turns out that $\omega=U_{r} / R$, where $U_{r}$ is the rolling speed and $R$ is the drop radius. We can now obtain a slip component by subtracting $y U_{r} / R$ from the drop profile, $U(y)$.

Figure 3.23 shows a velocity profile of a drop with its rolling and slipping velocity profiles extracted. The resulting slip profile is quadratic, and we can fit a quadratic profile (Eq. 3.23) to the slip profile to find an estimate for the slip speed. In computing that fit, we ignore data where $y \leq 1$ since data in this region are a known artifact of MD [1], where 1 represents one atomic distance. ${ }^{10}$ How we calculate the slip profile here is consistent with our theory and Eq. 3.23 for a drop purely sliding.

\footnotetext{
${ }^{10}$ This phenomenon arises from the L-J parameter $\sigma$, which is the characteristic spacing between atoms. In our case, the liquid and solid have $\sigma_{L S}=1$.
} 


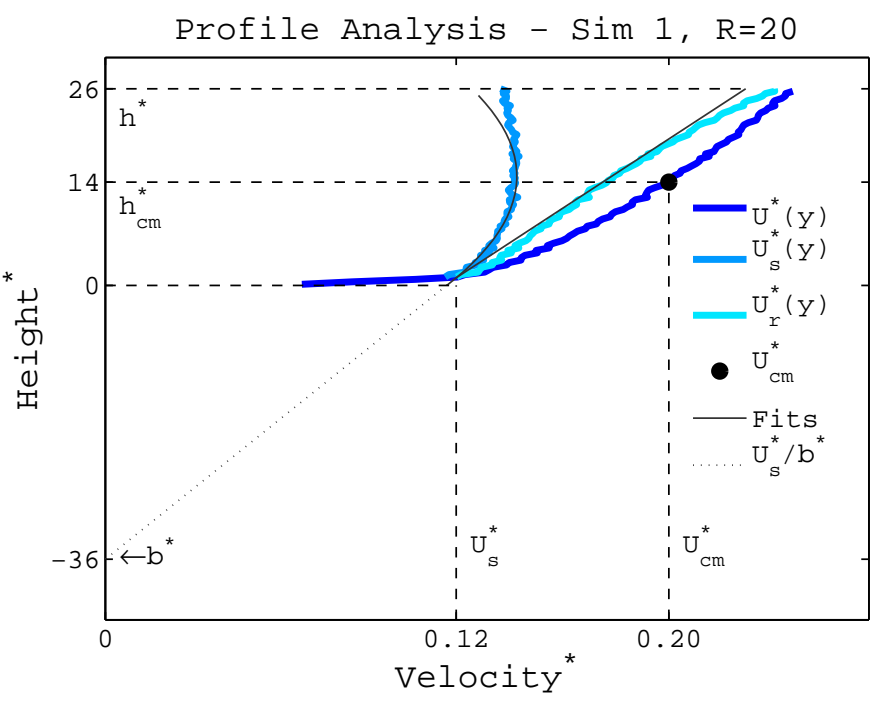

Figure 3.23: Extracting roll and slip. The drop's velocity profile can be broken down into its constituents, a linear roll profile and a quadratic slip profile. These profiles allow us to estimate $U_{r}^{*} / R$ and $U_{s}^{*} . h$ is the height of the drop and $h_{c m}^{*}$ is the height of its center of mass.

We estimate the slip speed, $U_{s}$, by evaluating the quadratic flow profile at $y=0$. Using the velocities recorded for the center of mass of the drop (Fig. 3.20) and Eq. 3.31, we find,

$$
U_{c m}=U_{s}+\frac{U_{r}}{R} h_{c m}
$$

where $U_{c m}$ is the speed of the drop and $h_{c m}$ is the height of the center of mass. For $R \ll \kappa^{-1}$ we expect $R=h_{c m}$. We included an estimation of the slip length in Figure 3.23 by taking the slope of the slip profile at zero. Slip lengths estimated from these flow profiles were consistent with estimates from Couette geometries. The slip lengths recorded in Fig. 3.20 are the averaged slip lengths from the drop profiles. A full analysis of all drop profiles is provided in Appendix C.

In Figure 3.24 we show $U_{r} / \alpha$ versus $R / \kappa^{-1}$ (left) and $U_{s} / \alpha$ versus $R / \kappa^{-1}$ (right) for all simulations. The data clearly show a $1 / R$ dependence for $U_{r}$ for simulations 1 to 4 . It is not clear to what the behavior in simulations 5 and 6 may be attributed; in these two simulations, gravity is much lower so it may be possible that rolling motion is being affected by the contact line dynamics. Also shown in the plot of $U_{r}$ is Eq. 3.21, which shows good agreement for drops with higher gravity. The results also reiterate the fact that rolling speed does not appear to be affected by the slip length.

On the right of Figure 3.24 we see $U_{s}$ approaching some limit. The data also verify the dependence of $U_{s}$ on slip length. The dashed lines represent Eq. 3.25 (color represents simulation number), and we see that this model predicts the the dependence of slip speed on drop size quite well; however, our model 

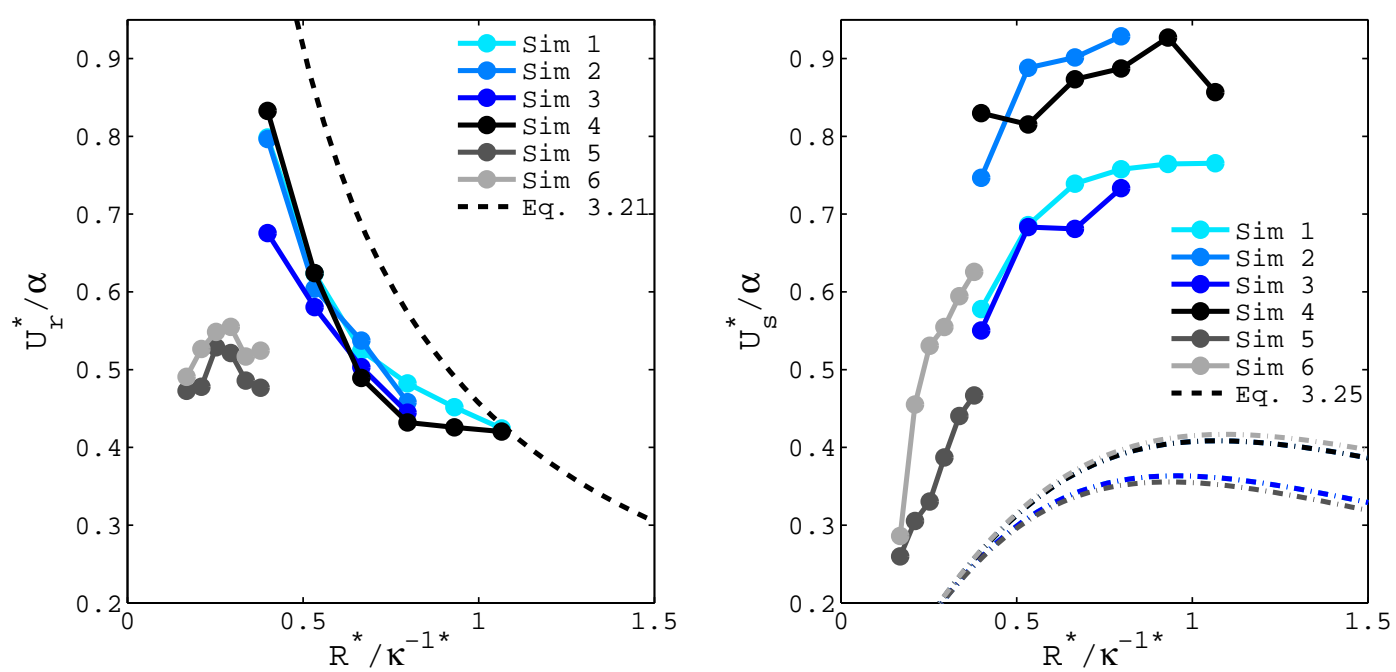

Figure 3.24: Rolling and slipping velocities. Estimated rolling and slipping speeds are plotted against scaled drop size (left and right, respectively). $U_{r}$ behaves as $1 / R$ and $U_{s}$ increases with drop size, approaching some limit. We plot Eq. 3.21 on the left and Eq. 3.25 on the right (--). The color of the dashed line represents the simulation number.

underestimates the slip speed by a factor of roughly 2.5. The model also fails to capture proper dependence on slip length as indicated by the closeness of the dashed lines.

\section{Comparing Rolling + Slipping Theory and Results}

Using Eq. 3.30 we can check our theory against our recorded values for $U_{c m}, U_{r}$, and $U_{s}$. On the left of Figure 3.25 we plot $U_{c m}$ scaled by $\alpha$ for the data $(\bullet)$ and for our theory (--), where we substitute our estimated rolling and slip speeds into our theory. We see that our model for dissipation compares decently with the data. For all simulations we more or less get the proper size dependence, increasing speed for Sims 5 and 6 and decreasing speed for Sims 1-4. On the right, we plot $U_{r}+U_{s}$ as given by Equations 3.21 and 3.25. We see that for Sims 1-4, our theory predicts the proper behavior of drop speed versus drop size. The theory doesn't seem to capture the effect of slip speed quite well as indicated by the closeness of the dashed lines. Seeing how our theory applied well in Figure 3.24 for Sims 1-4, it is no surprise that $U_{r}+U_{s}$ predicts similar behavior. Because our theory for $U_{r}$ didn't work well for Sims 5 and 6 , we don't get the proper behavior for drops at lower gravity. 

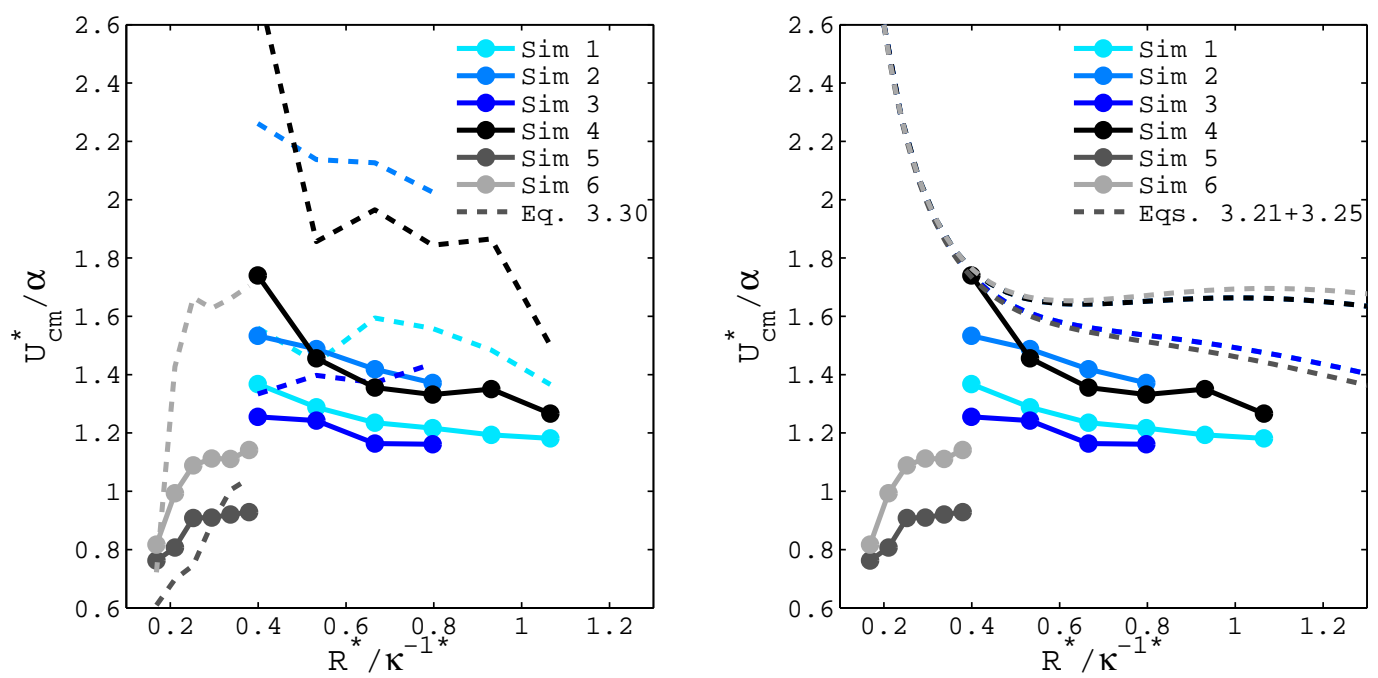

Figure 3.25: Theory versus data. This figure demonstrates the effectiveness of Eq. 3.30 in reproducing $U_{c m}$ given $U_{r}$ and $U_{s}$ (left). On the right we compare $U_{r}+U_{s}$ (Equations 3.21 and 3.25) with the data. The dashed line represents the theory, and the color of the dashed line represents the simulation number.

\subsubsection{Conclusion}

Through an extensive amount of simulations, we have shown the dependence of slip length and drop size on drop dynamics. As expected, larger slip lengths result in faster drops. In Sims 1-4 where viscous dissipation dominates, we see that drop speed decreases with $R$; however, as contact line friction becomes important, as in Sims 5 and 6, we can see drop speed increase with $R$. We found very little effect of slip length on rolling speeds, which is expected; in fact, this lack of slip length dependence suggests that shearing due to slip acts locally at the contact surface and does not extend up into the drop.

One of the most important results were the drop flow profiles. These profiles provided an indication of what was happening inside the drop, thereby providing insight on the types of dissipation that these drops encountered. More importantly, they showed how drops simultaneously slide and roll, and they allowed us to extract the contributions of these two types of motions.

We developed a model for sliding, rolling drops that compared reasonably well with our data. The model predicted proper size dependence for our simulations with higher gravity. When gravity was decreased by an order of magnitude, we no longer saw the same rolling behavior even though we saw the same slipping behavior. This could suggest that drop dynamics in the regime of a larger $\kappa^{-1}$ are dominated by forces other than those arising from viscosity. The dynamics of the contact line could be affecting rolling motion in this regime. 
Another possible cause for the poor agreement with data at higher $\kappa^{-1}$ is the fluctuations in the data recorded for these drops. Appendix $\mathrm{C}$ provides data on the drop speeds as a function of time. We saw fluctuations in the drop speed that had amplitudes larger than the difference between the averaged drops speeds at different sizes. We suspect that these fluctuations result from a combination of small gravity (small forces acting on the drop), surface geometry (post spacing on order of drop size), and possibly contact line dynamics (forces on order of $g$ ). Furthermore, for smaller drops as well as smaller angle of inclinations, drops may sometimes stick to the surface due to contact angle hysteresis. This effect is known to cause large fluctuations in drop speeds [22], and it would explain the fluctuations seen for both the large and small gravity simulations.

Our results have shed light on a specific aspect of moving drops that has not been well studied. The lack in data on rolling, slipping drops is primarily due to the difficulty in estimating slip and velocity profiles within millimeter sized droplets. Hopefully the data here will lead to further investigation. 


\section{Chapter 4}

\section{Graphene}

In this chapter, we will use MD to probe some of the properties of graphene. Whereas in the last chapter we detailed set up procedures and methods for calculating properties of a system, in this chapter we will use well proven methods so that we may focus on the simulation and results.

In Section 4.1, we will briefly introduce the potentials used to model graphene in MD. Then in Section 4.2 we will look at the energies of graphene and graphite using MD. These energies will provide an idea of the energies required to exfoliate graphene, a technique used to produce large amounts of graphene. Finally, in Section 4.3 , we look at graphene nano-bubbles on platinum and try to reproduce these bubbles using MD.

\subsection{Graphene in MD}

For our simulations of graphene energies, we will use the Adaptive Intermolecular Reactive Empirical Bond Order (AIREBO) which is known to provide an accurate description of carbon and hydrocarbon systems. Details of this potential can be found in the literature [34,35], and it is easy to implement in LAMMPS. We use the Embedded Atom Method (EAM) potential to model platinum and nickel in our graphene on metal simulations. EAM is a semi empirical model for metals and metal alloys, and it is computationally fast and accurate. Details on EAM may also be found in the literature [36].

Unfortunately, not many fast and accurate models are available for the interactions between graphene and metals. One group used the Modified Embedded Atom Method (MEAM) potential to accurately reproduce the energies of carbon atoms at various lattice sites on nickel as well as Ni-Ni and C-C bonds [37]. The potential was also formulated to model graphene, but it did not provide an 
accurate description of graphene. Another paper studied nickel and carbon nanotube composites with a MEAM potential, but this potential also did not model graphene well enough for our purposes [38] (graphene was not stable at high temperatures for these two MEAM potentials). One potential that is suitable for our study is the Reactive Force Field (ReaxFF), which is capable of producing models within accuracy of first principle calculations [39,40]. To formulate a ReaxFF potential, one starts from calculating the first principles quantum mechanics of a system and uses those results to train a reactive force field model. ReaxFF potentials for a graphene-Ni and graphene-Pt system are available from the literature [41,42]. The ReaxFF method is very computationally slow, so for our purpose of conducting a basic study of graphene on platinum and on nickel, we used Lennard-Jones potentials. The values for $\epsilon$ and $\sigma$ are $23.05 \mathrm{meV}$ and $2.852 \AA$ for Ni-C and $40.92 \mathrm{meV}$ and $2.936 \AA$ for Pt-C [43,44]. We don't expect the L-J potential to provide a good description of the metal-carbon interactions, but it will give us an indication of energies as well as a simplistic model of the effect of a metal substrate. 


\subsection{Graphene Energies}

\subsubsection{Motivation}

Knowing the energies required to peel off layers of graphene from graphite may help researchers looking to use the exfoliation technique. In chemical exfoliation experiments, it was shown that when the surface energy of graphene was balanced by the surface energy of a solvent, the enthalpy of mixing was low [45], making the separation of graphene from graphite energetically favorable. The surface energies of graphene were reported to be around $11-13 \mathrm{meV}$. Also important are the exfoliation energy, the energy required to peel graphene off of graphite, and the interlayer binding energy of graphite. A number of researchers made theoretical predictions for these values, but the predictions were not consistent. Recently Liu et al. experimentally measured the binding energy in highly oriented pyrolytic graphite (HOPG) to be around $44 \pm 3 \mathrm{meV} /$ atom [46]. Though precise measurements have yet to be made for these energy values, knowing their approximate values offers insight into making graphene. We will show that MD can reproduce with reasonable agreement the predictions and measurements of graphene energies.

We were also interested in graphene energies because we wanted to test a simple idea of using metal nano-particles in the exfoliation process of graphene. By comparing the energies of graphene on graphite with graphene on metal nanoparticles, we can test whether our simple idea would work. Basic MD studies like these demonstrate the value in using MD to help guide new ideas and experiments.

\subsubsection{Simulation and Results}

\section{Graphene and Graphite}

We simulated 1-5 and 9 layers of graphene using the AIREBO potential. Each layer consisted of 15,240 atoms $(100 \AA \times 100 \AA)$, and simulations were conducted at $300 \mathrm{~K}$ using a Nosé-Hoover thermostat. Each simulation ran for 25 picoseconds until potential energies reached equilibrium. Simulations were periodic in $x, y$, and $z$. Figure 4.1 provides snapshots of the six simulations as well as the recorded energies for each, and Figure 4.2 shows a top view of one simulation demonstrating the hexagonal close pack structure of multilayer graphene.

Figure 4.3 shows the convergence of the potential energies for each simulation (left) and the potential energy as a function of the number of layers (right). The data show that the energy per atom decreases as the reciprocal of the number of 


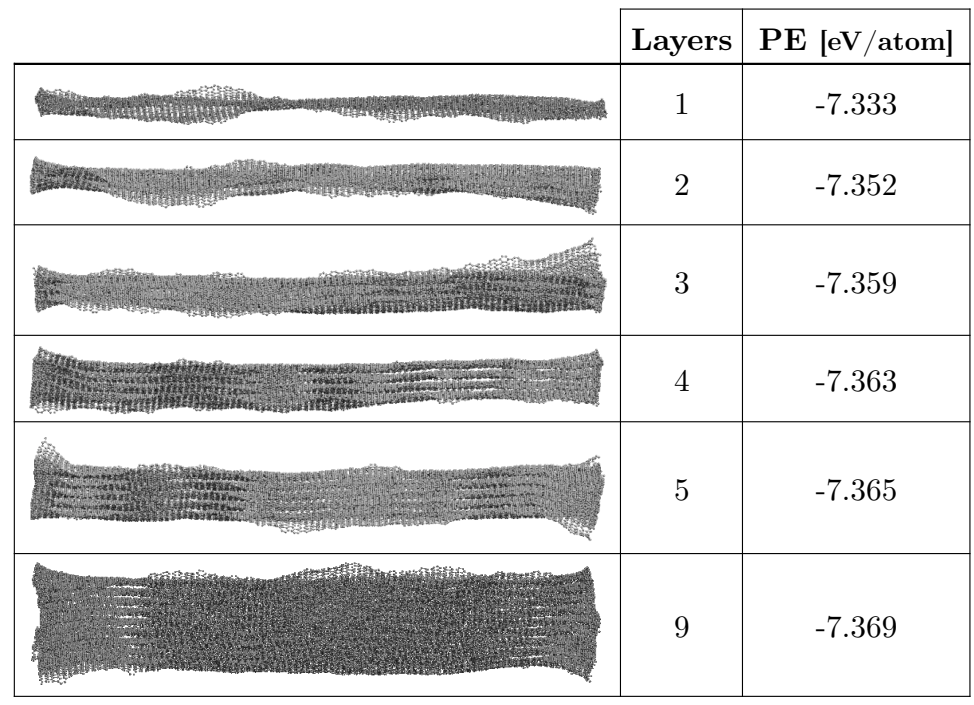

Figure 4.1: Graphene layers. Sheets of graphene from 1-5,9 layers were simulated, and the energy per atom was estimated for each.

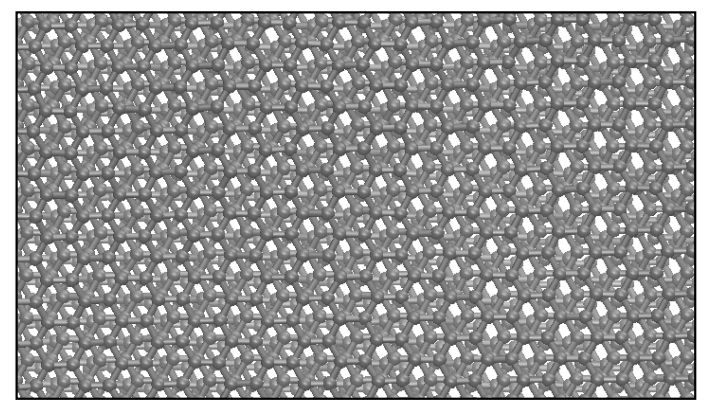

Figure 4.2: Stacking of graphene. A top view of a simulation with multiple layers indicating stacking of graphene sheets. 

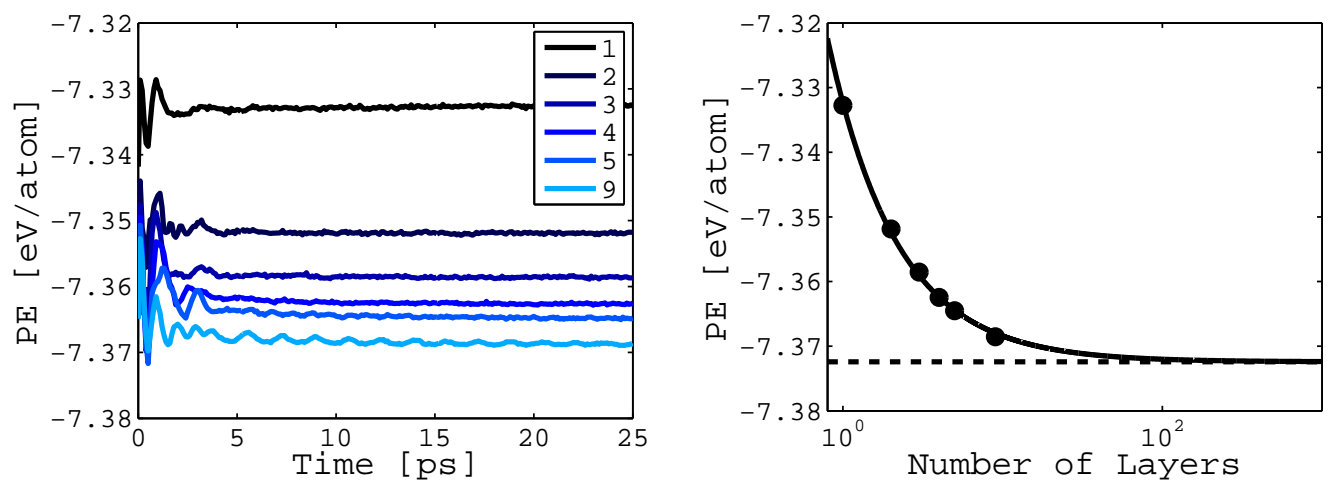

Figure 4.3: Graphene energies. Potential energy as a function of time for layers 1-5 and 9 (left) demonstrating equilibrium is achieved around 5 picoseconds. Potential energy as a function of the number of layers (right). Eq. $4.1(-)$ is fitted against the data $(\bullet)$ with good agreement. As the number of layers approach infinity, the energy approaches that of a graphite crystal (---).

layers. We found the curve,

$$
V=a L^{-1}+b
$$

provides a good description of the data where $L$ represents the number of layers and $a$ and $b$ are fitted parameters. For our data we have $a=40 \mathrm{meV}$ and $b=$ $-7.372 \mathrm{eV}$. The parameter $b$ should represent the energy per atom in HOPG because as the number of graphene layers approaches infinity, the system becomes more like graphite. If we consider the difference between $V(L=\infty)$ and $V(L=$ 1 ), we get $40 \mathrm{meV}$ which represents the amount of energy required to remove a single layer of graphene from HOPG (the exfoliation energy).

It is not possible to estimate an interlayer binding energy in HOPG given our data and Eq. 4.1. We would need to conduct a simulation where we explicitly separated two chunks of HOPG, which we did not do in this thesis.

\section{Graphene on Nickel}

We deposited graphene on the nickel (100) and (111) faces, where the nickel surface measured $110 \AA \times 110 \AA$ in length and width. Cubical nickel nano-particles of this size were synthesized [47] using particular reactants [48]. In our simulations, we simply created the nickel surfaces with sufficient depth for the MD cut-off radius. The simulation boundaries were fixed in $y$. Temperature, thermostatting, and graphene size were as above, and simulations ran for 115 picoseconds. Figure 4.4 shows snapshots of graphene on $\mathrm{Ni}(100)$ (left) and on $\mathrm{Ni}(111)$ (right). Notice the near perfect lattice match of graphene (light gray) on $\mathrm{Ni}(111)$ (dark gray) as expected [49-51]. The adhesion energies are $55 \mathrm{meV}$ and $61 \mathrm{meV}$ for (100) and (111). 

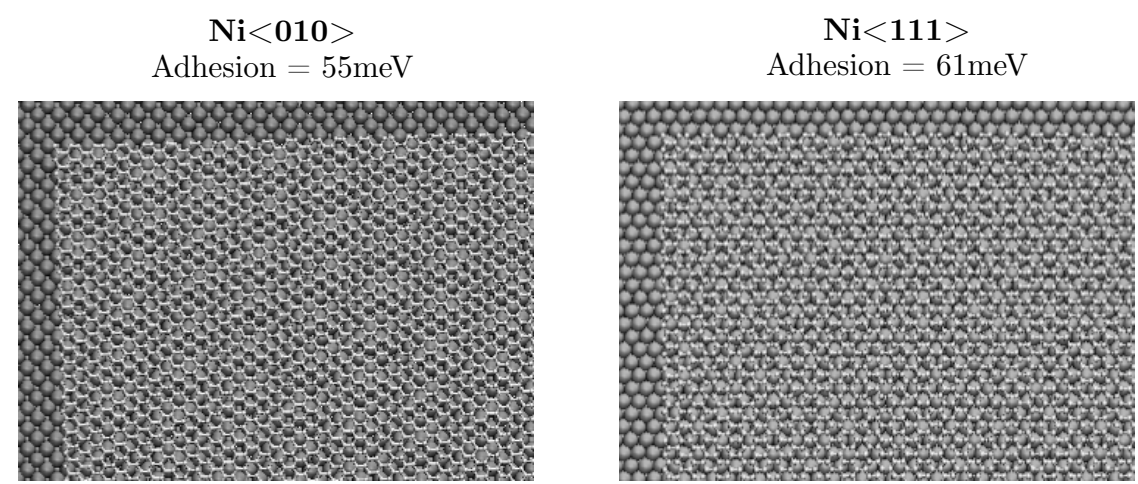

Figure 4.4: Graphene on nickel. Snapshots of graphene deposited on $\mathrm{Ni}(100)$ (left) and on $\mathrm{Ni}(111)$ (right) and corresponding adhesion energies. The lattice match between graphene and $\mathrm{Ni}(111)$ is near perfect as indicated by the moiré pattern.

Estimation of the binding energy of graphene on nickel is not very consistent [50], and depending on the lattice matching between graphene and $\mathrm{Ni}(111)^{1}$, variations in binding energy can be around $60 \mathrm{meV}$. The temperatures at which graphene is grown via chemical vapor deposition on nickel ranges from $753 \mathrm{~K}$ to $1273 \mathrm{~K}(65-110 \mathrm{meV})$, providing some indication of the energies required to achieve adhesion of graphene to $\mathrm{Ni}(111)$. In one DFT study of the adhesion energies of graphene on $\mathrm{Ni}$, the orientation with the strongest adhesion had an adhesion energy of $133 \mathrm{meV}$, while the lowest adhesion was $51 \mathrm{meV}$ [51]. Our simulation result of $61 \mathrm{meV}$ is within agreement of the low-temperature CVD experiments as well as the DFT study.

\subsubsection{Discussion and Conclusion}

Reported values for binding energy and exfoliation energy are around $24-50 \mathrm{meV}$, indicating that our relatively simple simulations produced energy values within reasonable agreement; however, the inconsistency of these recorded values was the motivation to accurately measure them [46]. One paper reports the exfoliation energy to be $43 \pm 5 \mathrm{meV}$ /atom with a binding energy roughly $18 \%$ larger [52], showing good agreement with our estimation for exfoliation energy, but these predictions contradict the experimental data unless the experimental data are incorrect or the difference between exfoliation energy and binding energy is small.

The surface energy for graphite is defined as the energy to overcome the van der Waals force when separating two sheets [45]. If we take the difference in the energies for simulations of monolayer graphene and two layer graphene, we find a surface energy of $20 \mathrm{meV}$. This value is higher than that reported but still within

\footnotetext{
${ }^{1}$ There may exist 3-6 different stable orientations of graphene on $\mathrm{Ni}(111)$
} 
reasonable agreement.

We found a simple model that represented the potential energy per atom as a function of the number of layers of graphene (Eq. 4.1), and the model fit the data very well. The model also allowed us to compute the estimated surface energy, exfoliation energy, and graphite energy, the former two showing reasonable agreement with the literature.

In our study of graphene on nickel, we found results consistent with the literature where graphene is more tightly bound to the (111) plane of the nickel lattice. When we compared the binding energies of graphene on nickel with that of graphene on graphite, we saw that graphene was more strongly bound to nickel. From a purely energetic argument and using our simple models, our results indicate that a nickel nano-particle could aide in the exfoliation of graphene from graphite. Thermodynamically, it would take $470 \mathrm{~K}$ to exfoliate graphene from graphite, $640 \mathrm{~K}$ to peel graphene from the $\mathrm{Ni}(100)$, and $710 \mathrm{~K}$ to peel graphene from $\mathrm{Ni}(111)$.

The simplicity of our simulations and the availability of the tools used in our simulations highlight the applicability of MD to produce reasonable results to guide researches in designing experiments and testing simple models. 



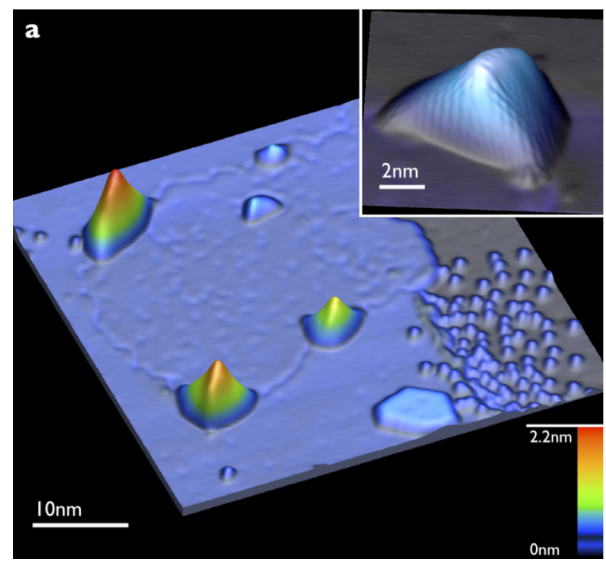

Figure 4.5: Graphene nano-bubble on platinum. [53]

\subsection{Graphene Nano-Bubbles}

\subsubsection{Motivation}

Of the many fascinating characteristics of graphene, nano-bubbles are one feature which may promise novel nano-electronic devices. Levy et al. made an interesting discovery when growing graphene on platinum [53]. They found nano-bubbles in their graphene samples that were as tall as $2 \mathrm{~nm}$, and they also found that the strain in these bubbles created local magnetic fields in excess of 300 Tesla as well as local scalar potentials. These measurements were consistent with theory, which predicts that strain will change the electronic structure of graphene. The large magnetic fields confirmed in strained graphene could lead to new types of research, and these experiments demonstrate the ability to strain-engineer graphene devices.

Levy et al. were not certain on the direct cause of the nano-bubbles. Most bubbles formed at the edges of the graphene substrate, though sometimes they would find bubbles in the middle. They suggested that one possible cause was the differing thermal expansion between platinum and graphene. In their experiment, they grew graphene by exposing the platinum (111) face to ethylene gas at $1170 \mathrm{~K}$, and then they quenched their samples down to $7.5 \mathrm{~K}$. Though platinum will contract under these conditions, graphene is known to expand upon cooling $[54,55]$. Graphene is also weakly coupled to platinum as opposed to other transition metals, but the stability of the bubbles suggests that graphene must be pinned to the platinum substrate along the perimeter of the bubbles.

In this section, we will use MD to create bubbles in graphene. These simulations will hopefully provide some insight into the primary causes for the formation of graphene nano-bubbles. 


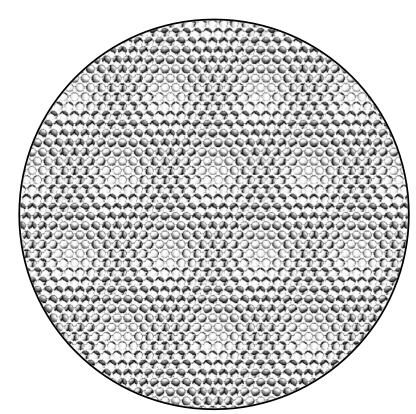

Figure 4.6: Graphene on platinum. The moiré pattern of graphene on platinum demonstrates the mismatch in the lattices, which is about $11 \%$.

\subsubsection{Cause for Bubbles}

The thermal coefficient of expansion, $\alpha$, of a material is measured as, $\alpha=$ $L^{-1} d L / d T$, where $L$ is the linear dimension, and $d L / d T$ is the differential change in $L$ per change in $T$. Typically, $\alpha$ varies for different temperatures, and one can find the total percent expansion/contraction by integration. For our simulations, we will be considering $\Delta T=7.5 \mathrm{~K}-1000 \mathrm{~K}$, and from the literature we find that this represents a $0.9 \%$ contraction of platinum [56] and a $0.3 \%$ expansion of graphene [54]. If we assume graphene is pinned to platinum at $1000 \mathrm{~K}$, then these differing thermal expansions can result in $1.2 \%$ total strain in the graphene lattice.

The (111) face of the fcc lattice structure provides a template for graphene growth. The platinum lattice constant is $3.92 \AA$, and, on the (111) face, the nearest neighbor atom distance is about $1.6 \AA$, not much larger than $1.42 \AA$ for graphene. This lattice mismatch could potentially produce $11 \%$ strain in graphene. That additional strain could be the cause of bubble formation. Figure 4.6 shows a sheet of graphene on $\mathrm{Pt}(111)$. The light colored regions are where the two lattices align.

\subsubsection{Simulation}

We conducted a series of simulations to try and understand the bubbling of graphene on platinum. We use the AIREBO potential for the $\mathrm{C}-\mathrm{C}$ interactions, the EAM potential for the Pt-Pt interactions, and the L-J potential for the C-Pt interactions.

If you simply compress a sheet of graphene, the out of plane shape that emerges is dependent on the boundary conditions of the sheet. For example, if you held a circular set of points on a graphene lattice fixed (in-plane) and compressed that circle inwards, then you would end up with a nano-bowl [57]. The shapes found in nano-bubbles are therefore dependent on the type of pinning 
at the boundary. The bubbles produced by experiment were of triangular shape, so we used a triangular shaped piece of graphene to mimic experiment.

In our first set of simulations, we compressed triangular sheets of graphene. The edges of these sheets remained fixed in-plane while they were contracted inwards. The rest of the graphene sheet was allowed to relax in all dimensions. Figure 4.7 shows a graphene sheet with the fixed boundary highlighted in red. The arrows indicate the direction of contraction for all carbon atoms in the boundary. This boundary thickness remained constant at $7.1 \AA$ while the sheet size was varied.

For our second set of simulations, we changed the boundary conditions of the graphene sheets. Rather than having rigid edges, we attached each atom in the boundary to springs. We looked at three different sheets sizes $(676,841$, and 1024 atoms) and three different spring strengths (1, 10, and $100 \mathrm{eV} / \AA)$. In LAMMPS, we can apply a spring to an atom based on the atom's initial position. Any displacement of the atom from that position results in a repulsive force, $\mathbf{F}_{\mathbf{k}}=-k \mathbf{r}$, where $\mathbf{r}$ is the radial displacement of the atom from its origin. For our simulations, we only allowed relaxation in the $y$ plane, so $\mathbf{r}=y$. The values for $k$ were chosen to cover a wide range of binding energies and are not indicative of actual binding energies of atoms to the substrate.

Finally, we conducted two simulations where we contracted graphene as in our first sets of simulations but placed the graphene on a platinum substrate. The rigid boundary of the graphene flake was treated as a rigid body which was allowed to move along the surface of the platinum substrate. This boundary condition allowed the graphene flake to find an equilibrium location on the $\operatorname{Pt}(111)$ surface relative to its current geometry. Figure 4.7 shows a snapshot of a graphene flake (gray) on platinum (white).

All simulations were carried out at $7.5 \mathrm{~K}$ to mimic conditions at which the nano-bubbles were observed. Simulations ran for 30 picoseconds at each percent contraction in order to allow bubbles to equilibrate. Simulations were periodic in $x, y$, and $z$.

\subsubsection{Results}

\section{Graphene with rigid boundaries}

As expected, nano-bubbles were formed upon squishing sheets of graphene. Figure 4.8 shows the potential energy and the maximum bubble height of the sheets of graphene versus the percent contraction of the boundary . Larger sheets are ca- 


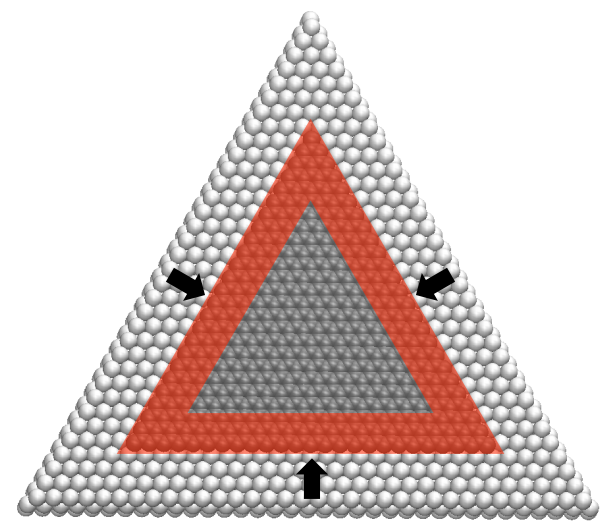

Figure 4.7: Squishing graphene. A triangular graphene flake (gray) is squished by compressing the boundary, highlighted in red, inwards, as indicated by the arrows. In some simulations, the graphene was placed on a platinum substrate (white).
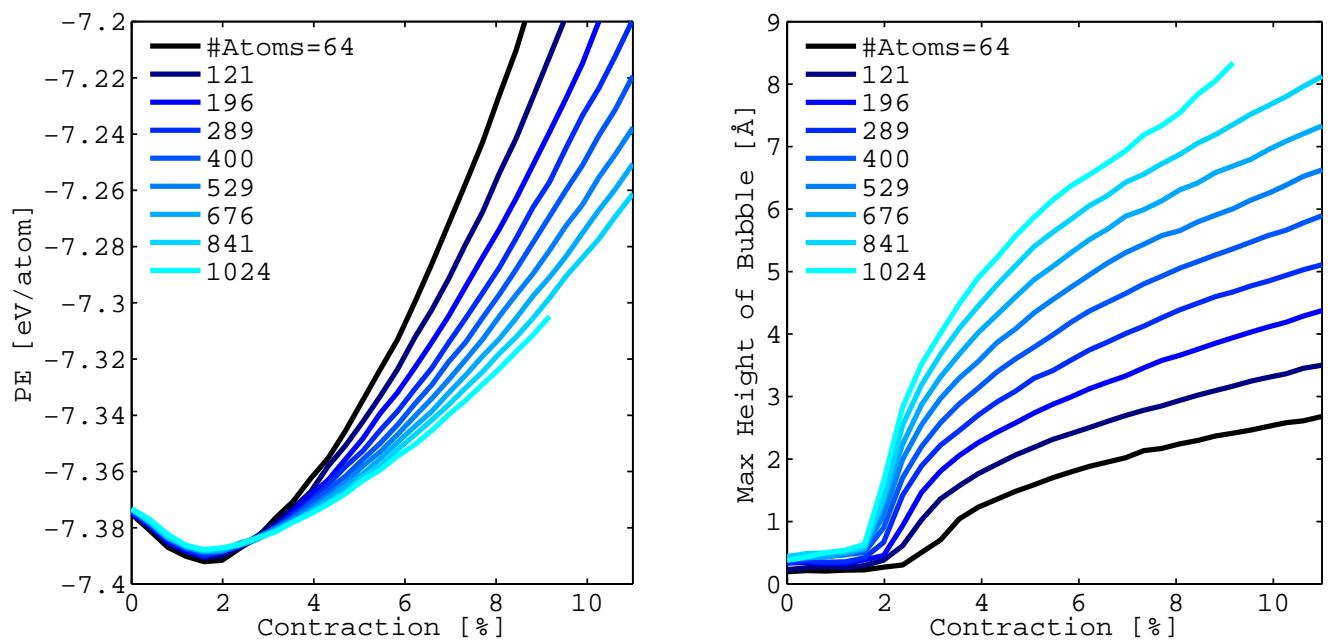

Figure 4.8: Bubble results. The potential energy (left) and the maximum height (right) of a bubble versus percent contraction of the boundary. The boundary is not included in the atom counts or the potential energies.

pable of producing taller bubbles, which is geometrically expected, but the larger surface area means that strain will be smaller within the sheet. This is evidenced in the decreasing energy profiles with increasing sheet size. Interestingly, there is a small decrease in energy upon contracting the boundary from $0-2 \%$, and this corresponds to the lack of bubbling up to $2 \%$. Then, the graphene begins to bubble above $2 \%$ and its potential energy starts to increase.

The energy minimum at roughly $2 \%$ indicated that our graphene sheets were not initially relaxed. This makes sense because free standing graphene will ripple (see Figure 4.1), indicating that the relaxed state of graphene is not a flat one. This implies that we are actually creating strain in the graphene by holding its edges flat, and by compressing the edges we relieve this initial strain. 


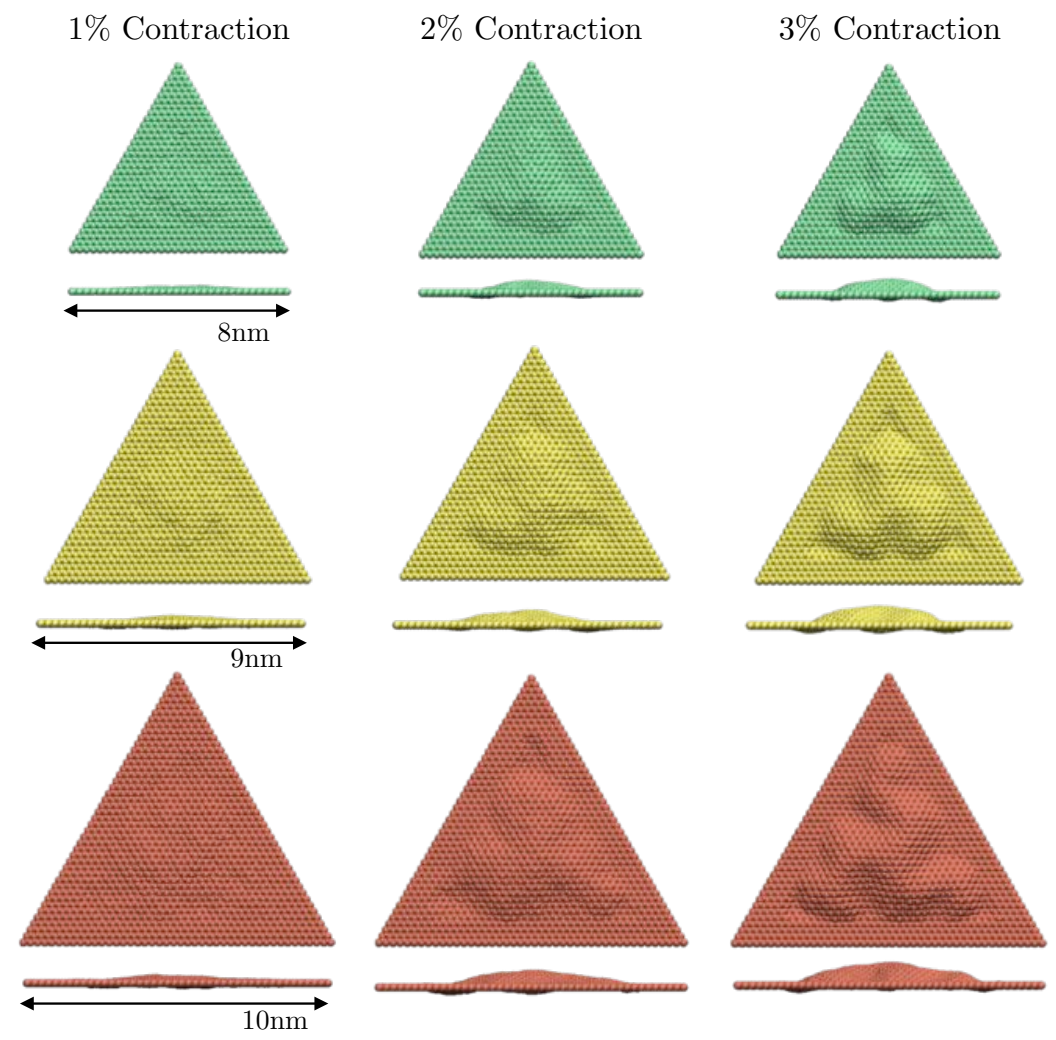

Figure 4.9: Bubbles with rigid boundaries. Snapshots of graphene bubbles of size 400, 676 , and 1024 atoms for 1-3\% contraction. Short bubbles appear relatively smooth (center), but the bubbles begin to ripple at larger contractions (right).

Snapshots of graphene sheets of size 400, 676, and 1024 atoms are shown in Figure 4.9 for $1-3 \%$ contraction. At $3 \%$ we have a height of roughly $4 \AA$ for the large sheet, similar to the heights of the bubbles seen by Levy et al. One primary difference with our bubbles is the bubble shape. Whereas the experiments had smooth bubbles, our bubbles appeared to have ripples. ${ }^{2}$ The ripples became more prominent as we further contracted the substrate, and this effect was likely due to our boundary conditions.

\section{Graphene with soft boundaries}

Turning the rigid boundary condition into a soft one made of springs had little effect on the smoothness of our bubbles, as seen in Figure 4.10. Although we do see a relatively smoother bubble for $k=10 \mathrm{eV} / \AA$, we still see a few ripples for $k=1 \mathrm{eV} / \AA$. The boundaries also show signs of rippling, and the period of these ripples is proportional to the atom spacing.

Figure 4.11 shows the energies (left) and heights (right) of the bubbles. The

\footnotetext{
${ }^{2}$ Theoretical calculation of the magnetic fields in the bubble compared extremely well with experimental measurements, confirming the smooth geometry of the bubbles [46].
} 

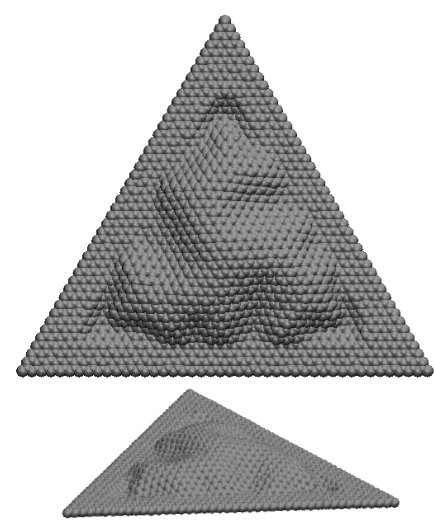

$\mathrm{k}=100 \mathrm{eV} / \AA$
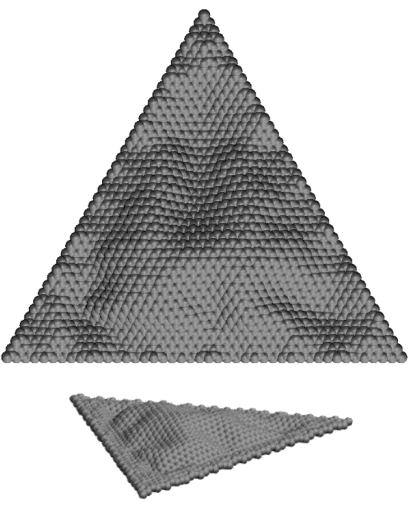

$\mathrm{k}=10 \mathrm{eV} / \AA$
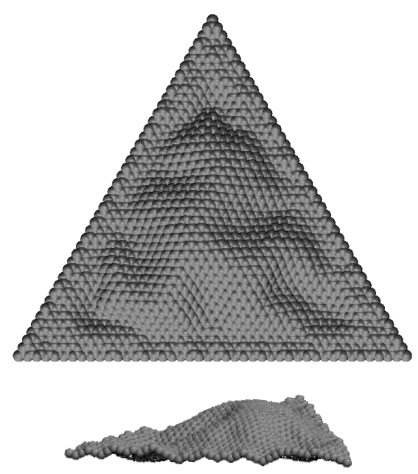

$\mathrm{k}=1 \mathrm{eV} / \AA$

Figure 4.10: Bubbles with soft boundaries. Snap shots of graphene bubbles with soft boundaries, where $k$ is the spring constant pinning the boundary atoms to the y-axis. The results show that there is little change in the bubble's smoothness when we changed the boundary conditions and that the springs on the boundary atoms induce ripples along the boundary.

solid line represents a rigid boundary, and the dash, dash-dot, and dotted lines represent springs with $k=100,10$, and $1 \mathrm{eV} / \AA$, respectively. Energy curves were shifted by $50 \mathrm{meV}$ and height curves were shifted by $1 \AA$ for clarity. In this figure we see that softer boundaries lower the potential energy of the graphene sheets. This makes sense as the springs would lower the local stress at the boundary. We also see a slight increase in bubble height with decreasing spring strength.

\section{Graphene on platinum}

We placed graphene sheets consisting of 256 and 1722 atoms (not including boundary atoms) on platinum substrates. Results of these simulations are shown in Figure 4.12. Detailed data of graphene energies and height were not collected for these simulations, so we provide visual results.

We noticed that the platinum substrate lowers the height of the bubbles. Sheets of graphene on platinum must be contracted further in order to see comparable heights with the other graphene sheets. For example, for the smaller sheet at $4 \%$ contraction we only see a maximum bubble height of about $1 \AA$ whereas a comparable stand-alone sheet would have a bubble with a maximum height of about $2.5 \AA$ (Fig. 4.8). We also noticed that the platinum substrate creates more pronounced ripples in the graphene sheets, and these ripples are typically $10 \AA$ in width. 

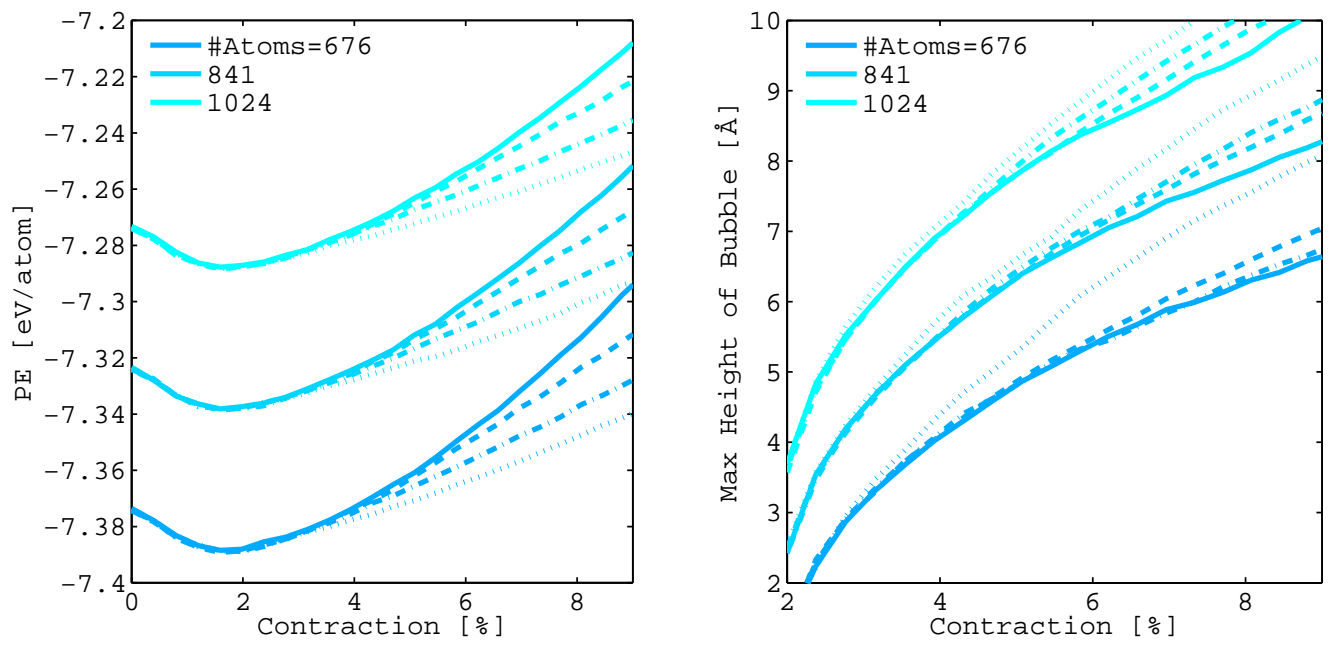

Figure 4.11: Bubble energies. Potential energy (left) and height (right) curves for bubbles with springs on the boundary. Curves for sheet sizes of 841 and 1024 atoms are shifted by $50 \mathrm{meV}$ and $100 \mathrm{meV}$ on the right and by $1 \AA$ and $2 \AA$ on the left. Dash, dash-dot, and dotted lines represent boundaries with springs of $k=100,10$, and $1 \mathrm{eV} / \AA$, respectively, and the solid line represents the rigid boundary condition.

\subsubsection{Discussion and Conclusion}

Our results showed that bubble formation in free-standing graphene was not prominent until the sheet was contracted by more than $2 \%$; however, we noticed that at $2 \%$ we had an energy minimum. This may imply that $0 \%$ contraction is actually at $2 \%$. If this is the case, then the differing thermal coefficients of expansion between graphene and platinum, $1.2 \%$ strain, could potentially produce bubbles as tall as the ones seen in experiment. It is important to note that when a sheet of graphene is placed on a substrate, the graphene will flatten. This flattening, in addition to any pinning around the edges, will create strain in these graphene sheets. It may well be the case that graphene will initially relax when contracted on a substrate until a point where strain begins to increase. We did find that softer boundary conditions created taller bubbles, but they were only significantly taller when the sheets were contracted by more than $5 \%$. These results suggest that other causes such as lattice mismatch and pinning must also contribute to bubble formation.

When we placed graphene on platinum, we saw that bubble height decreased, more contraction was required to create bubbles, and the sheets had more ripples. These effects are certainly due to the Lennard-Jones interaction between the two materials, and it may be possible that the C-Pt interaction is too strong. Another aspect of our model that may affect results is the height of the graphene boundary above the platinum substrate. A pinned $\mathrm{C}$ atom would sit more closely to the $\mathrm{Pt}$ 


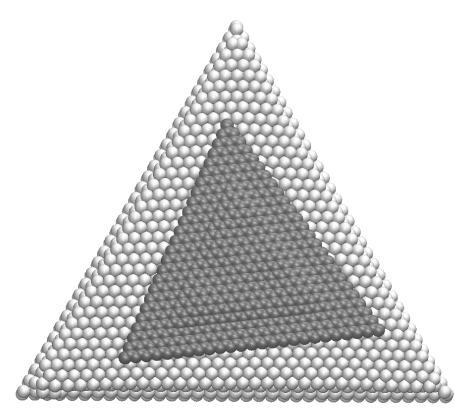

$4 \%, h=0.8 \AA$

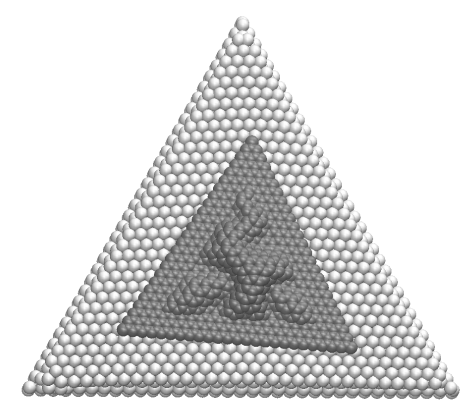

$14 \%, h=5.1 \AA$

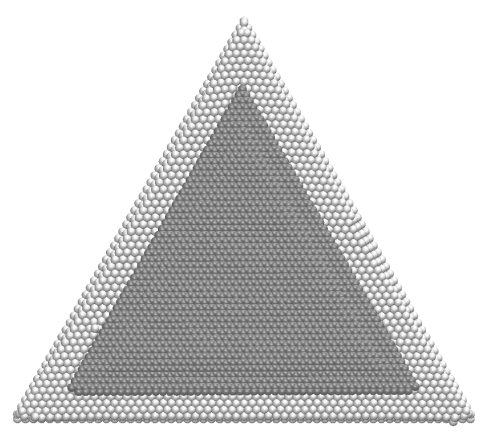

$2.5 \%, h=0.7 \AA$

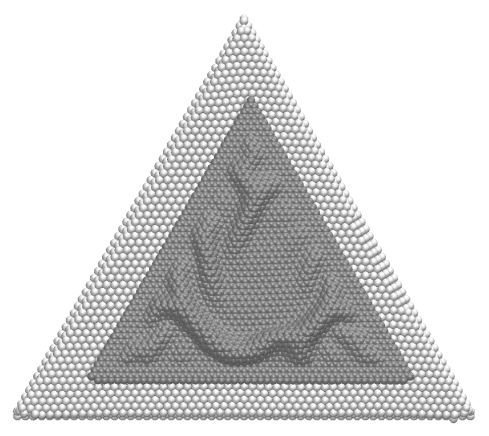

$10 \%, h=5.2 \AA$

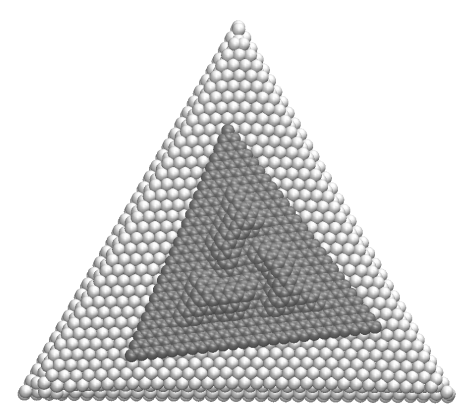

$7.5 \%, h=2.3 \AA$

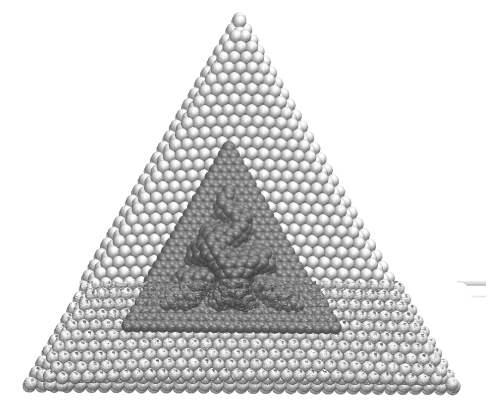

$22 \%, h=7.0 \AA$

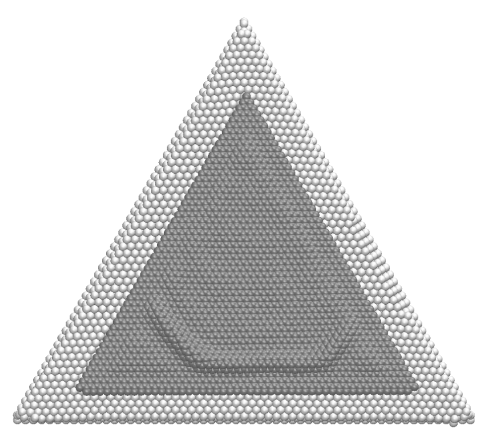

$5 \%, h=3.0 \AA$

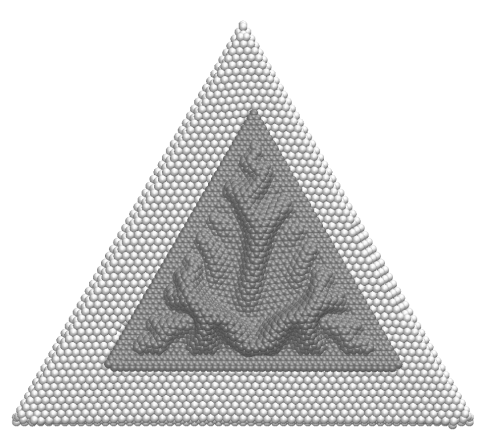

$18 \%, h=9 \AA$

Figure 4.12: Graphene on platinum. Above are simulation snapshots at various boundary contractions. The top four snapshots have 256 atoms in the unconstrained portion of the sheet, and the bottom four snap shots have 1722. $h$ is the maximum bubble height. 


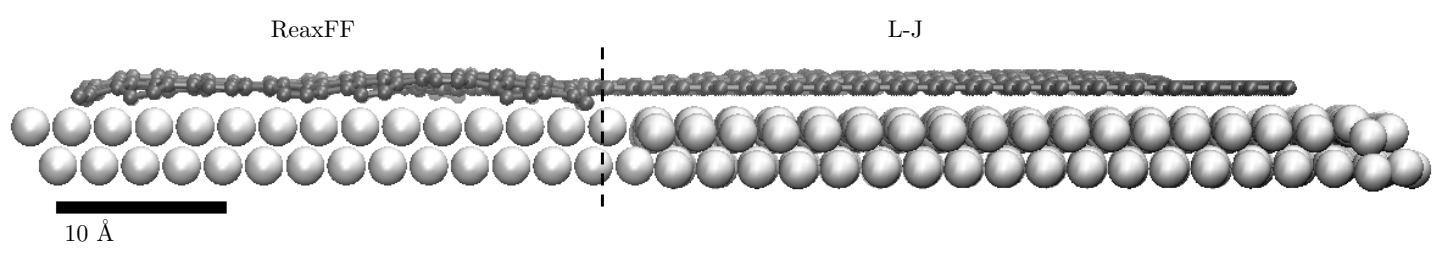

Figure 4.13: ReaxFF potential. A simulation of graphene on Pt using the ReaxFF (left) and the L-J (right). On the left we see pinning of $\mathrm{C}$ atoms at the edges of the graphene sheet, and we see possible beginnings of bubble formation as evidence by the slight protrusion of the graphene flake. The boundaries of the L-J simulation (right) do not allow pinning of $\mathrm{C}$ atoms closer to Pt atoms; instead, these edge atoms sit at the equilibrium height of the L-J potential.

substrate, and this height differential could cause protrusions. This would explain why bubbles were more commonly found at the edges of the graphene flakes. Our models lacked this height differential at the boundary, and perhaps including this in the model could possibly lead to better bubble formation and less ripples. Our simulations with springs on the boundary support this conclusion. The springs allowed the boundary atoms to move closer to the substrate, and we observed one instance where this slightly improved bubble formation $(k=10 \mathrm{eV} / \AA)$.

We conducted a small simulation using the ReaxFF potential, and a snapshot of a graphene sheet deposited on platinum is provided in Figure 4.13, where we have the ReaxFF model on the left and the L-J model on the right. For the ReaxFF potential, not only do we see pinning around the boundary as evidenced by the spacing between $\mathrm{C}$ and $\mathrm{Pt}$ atoms, but also we see what could be the beginning of bubbles. This bubbling may be due to the height differential, and it may also be due to a weaker interaction between graphene and platinum. No artificial strain was included in this ReaxFF model, and the simulation was performed at $300 \mathrm{~K}$ while the platinum substrate was fixed.

Our work has shown that mimicking nano-bubble formation in MD is difficult. It may well be that the potentials we used in our models are not satisfactory, and if we want more informative results we may have to use more accurate models such as the ReaxFF. Nevertheless, we can conclude that an MD model which properly considers pinning of graphene to the substrate as well as the location of the pinning with respect to the lattice mismatch between graphene and platinum ought to produce bubbles more in line with experiment. 



\section{Chapter 5}

\section{Conclusion}

We have demonstrated the power of MD to study systems as different as viscous liquids and graphene. Theoretically, MD is capable of modeling any physical system consisting of matter, but some systems may be difficult to study because their force models may not be well understood or their models are too computationally expensive.

The simple Lennard-Jones potential is an excellent model to test systems in MD. It is computationally fast, and it even predicts properties of the noble elements like Argon very well. The two parameters in the L-J potential, $\epsilon$ and $\sigma$, allow us to easily scale between different systems, especially when we work in dimensionless units.

We can also treat MD like an experimental tool where we conduct experiments in simulation. We can use models that accurately represent real materials such as the AIREBO and ReaxFF models. Though these models may not capture the precise behavior of a system, they can provide good approximations. They give us a general idea of the parameters we need to consider, and they can aid us in designing new experiments, be it in the lab or in simulation.

\section{$5.1 \quad$ Liquids}

We started Chapter 3 by calculating properties of our L-J liquid. Because L$\mathrm{J}$ liquids are well studied, we had data with which to compare our calculations. Using models found in the literature, we were able to estimate the density, surface tension, and viscosity of our L-J liquid. One of the challenges of calculating properties of L-J liquids was the existence of a vapor phase. We had to separate vapor from liquid in order to get proper estimates, and we developed a method that was successful in separating the two. Our method looked at the radial 
distances of atoms from the center of a liquid drop. We found that the criteria $R \Delta R>1$, which is analogous to looking at the number of atoms on a spherical shell, provided an efficient way to determine which atoms were liquid and which were vapor without having to bin atoms. With this method, we were able to calculate density, surface tension, and viscosity, all within good agreement with the literature.

\subsubsection{Effective Slip}

Our first task was to estimate the effective slip length of a mixed surface made up of smaller surfaces. A recent theory was developed [20] that estimates effective slip given the intrinsic slips and geometries of a surface, and we sought to confirm this theory in simulation. We created our surfaces using a simple cubic lattice, and we varied the slip length of the surface by changing the $\epsilon$ parameter in the L-J potential between the liquid and the surface atoms. The surfaces for which we wanted to estimate an effective slip were made up of two surfaces with different $\epsilon_{l s}$. We also added roughness to our surfaces by simply scooping out atoms in the lattice. Interestingly, our method of creating our atomic sized surface resulted in a third surface, the (111) face of the simple cubic.

We had some difficulty in simulating Couette flows and estimating slip length because our liquid sometimes froze as a result of high viscosity and ordering near the liquid-surface interface. We were able to alleviate this issue by using smaller gravitational forces and by creating a soft driving plate with which to move the liquid. Our estimates of the effective slip length, $b_{\text {eff }}$, matched very well for the theory for one set of simulations. For the other two sets of simulations, the theory showed similar behavior with regard to the mixing of slip. Predominately evident in both the theory and our simulations was the fact that the lowest intrinsic slip length, arising from the (111) face, dominated the effective slip.

Some of the slip lengths we estimated in the mixed surfaces were not very consistent with the intrinsic slip lengths. In one set of simulations, all the rough surfaces had lower slip lengths than the (111) face. We assumed that this could be an incorrect estimate of the (111) face. We investigated more closely the (111) simulations by looking at snap shots and density profiles of the liquid-surface interface, and we could not make any good conclusions as to why we may have poorly estimated the slip length. Perhaps our estimates were accurate, so it may be possible that surface roughness was dominating the slip and that the intrinsic slip had little effect. 


\subsubsection{Rolling, Slipping Drops}

Our next simulation was to study the rolling and slipping motion of drops moving down a super-hydrophobic incline. We used a surface patterned with posts to create super-hydrophobicity, and we varied the $\epsilon_{l s}$ parameter to change slip length. We considered different angles of inclinations for the surface, and we also considered different forces of gravity. Changing gravity was mainly done to allow us to study drops small enough such that $R<\kappa^{-1}$.

We performed many simulations, and we investigated drop speeds in addition to the velocity profiles within the drops. We found results consistent with our expectations: drop speeds increased with larger slip lengths and angles of inclinations, and rolling speeds decreased with increasing drop sizes. The scaling laws that we developed were meant to predict the behavior of drops with respect to drop size, slip length, and the liquid properties (namely surface tension and viscosity). We found that the theory for purely rolling drops matched our rolling speeds well. Our theory for the purely sliding case gave the same dependence of drop speed on size, but the theory underestimated speeds by a factor of about 2.5 .

Viscous forces in the volume of the contact zone, shear at the surface of the contact zone, and contact line friction on the perimeter of the contact zone all serve as methods of energy dissipation. These dissipations, when balanced with the change in gravitational potential energy, are the core of our theory for predicting drop speed. Our theory for $U_{c m}$ provided the proper behavior of drop speed with drop size for our simulations where $\kappa^{-1}$ was smaller. Even considering dissipation and our extracted rolling and slipping velocities, our theory gave us the correct size dependence for both large and small $\kappa^{-1}$. Our theory did not produce the rolling behavior we observed for Sims 5 and 6 . We mentioned that this abnormal behavior could possibly be due to the fluctuations arising from our low gravity as well as contact line dynamics. One way to mitigate these fluctuations would be to create a surface where post spacing is much smaller than drop size.

Though our models did not perform as well as we would have liked them to, the data we've collected on rolling, slipping drops provided insight into drop dynamics on super-hydrophobic surfaces. 


\subsection{Graphene}

We studied basic properties of graphene in Chapter 4 using models found in the literature. We were interested in graphene energies because they provide some insight into the manufacturing of graphene. We were also interested in reproducing graphene nano-bubbles in MD to try and understand the causes of the bubble formation.

Using the AIREBO potential, we simulated layers of graphene and calculated their energies. We found that the per-atom energies of the graphene layers increased as the inverse of the number of layers. With this simple behavior, we were able to estimate the energy of graphite (HOPG to be specific). This then allowed us to calculate the energy required to exfoliate a single sheet of graphene from graphite. We found $40 \mathrm{meV}$ for the exfoliation energy, which was consistent with other theoretical and numerical findings. We also looked at the energies of graphene on nickel using a simple L-J potential, and our results allowed us to estimate the types of energies that may be required to deposit and exfoliate graphene from nickel nano-particles. Consistent with the literature was the fact that the (111) face had a stronger binding energy to graphene than the (100) face, indicating that the simple L-J potential can provide useful results.

Our last study looked at the formation of graphene nano-bubbles on platinum substrates. For the sake of computation time, we had to use the L-J potential to model the C-Pt interactions. We started our study by creating graphene flakes that had a geometry similar to the bubbles in the experiment. We held the edges of the flake fixed and we squished the edges inward. This method with rigid boundary conditions produced bubbles similar to those found in experiment. We noticed that the potential energy of the graphene flakes initially decreased upon contraction. This trait is likely due to the pre-strain created in graphene by flattening the sheet and giving it rigid edges - a free-standing graphene sheet is rippled in its relaxed state.

To try and simulate a more realistic model of graphene nano-bubbles, we changed our boundary conditions and attached the edges of the graphene sheets to springs. This soft boundary condition helped to create slightly larger bubbles, and it also provided a closer representation of pinning at the edges.

Finally, we placed sheets of graphene on $\mathrm{Pt}$ and contracted the sheets to try and form bubbles. We found that the Pt substrate hindered bubbling, and our graphene sheets required larger contractions in order to produce bubbles with heights similar to our free-standing bubbles. We also found that the $\mathrm{Pt}$ substrate created rippled bubbles, where the ripples were on order of $10 \AA$ in 
width. These effects were likely due to the strong L-J potential, whereas graphene is known to bond relatively weakly with Pt. We conducted a small study of graphene on Pt using the ReaxFF potential, and we found much better results. We saw pinning at the edges as well as the beginnings of bubble formation prior to any contractions. We concluded that properly modeling pinning and the weak graphene-Pt interaction are important when trying to create nano-bubbles in MD. 



\section{Appendix A}

\section{Liquid Calculations}

Table A.1 provides drop atom counts along the various thresholds used to separate liquid from vapor. The table also provides the estimated drop radius and density.

The data provided are averaged over 302 time units with a time step of $1 \times$ $10^{-3}$. Data were logged every 1 unit of time, so data are averaged over 302 points. The size of the simulation box varied based on the number of atoms to improve computation time. An NVE integration was used, and a simple velocity scaling thermostat was used where $T^{*}=1 \pm 0.02$. Velocities were rescaled every $10^{3}$ time steps if $T^{*}>1.02$ or $T^{*}<1.02$. 
Table A.1: Liquid drop calculations. $N$ is the number of atoms in the simulation, $N_{V_{T}}$ is the number of atoms that meet the potential energy threshold $V_{t}^{*}, N_{R}$ is the number of atoms that meet the energy threshold as well as the radial threshold, $R^{*}$ is the approximate radius of the drop (radial threshold), $V^{*}$ is the potential energy of the drop, $V_{\text {liq }}^{*}$ is the potential energy of the drop after the energies of the vapor regions were removed, and $\rho^{*}$ is the estimated density.

\begin{tabular}{|c|c|c|c|c|c|c|}
\hline$N$ & $N_{V_{t}}$ & $N_{R}$ & $R^{*}$ & $V^{*}$ & $V_{\text {liq }}^{*}$ & $\rho^{*}$ \\
\hline \hline 43 & 42 & 3.4 & 39 & -199 & -180 & 0.790 \\
55 & 52 & 3.8 & 50 & -263 & -234 & 0.816 \\
87 & 83 & 4.2 & 80 & -481 & -418 & 0.832 \\
141 & 137 & 4.8 & 134 & -885 & -766 & 0.840 \\
201 & 194 & 5.2 & 190 & -1330 & -1153 & 0.853 \\
249 & 242 & 5.6 & 238 & -1716 & -1500 & 0.856 \\
369 & 359 & 6.0 & 355 & -2697 & -2368 & 0.862 \\
459 & 445 & 6.6 & 440 & -3417 & -3033 & 0.861 \\
603 & 588 & 6.9 & 584 & -4655 & -4138 & 0.862 \\
683 & 660 & 7.3 & 656 & -5287 & -4731 & 0.863 \\
935 & 908 & 7.9 & 900 & -7458 & -6745 & 0.864 \\
1061 & 1028 & 8.1 & 1021 & -8528 & -7723 & 0.864 \\
1289 & 1257 & 8.7 & 1250 & -10597 & -9678 & 0.863 \\
1505 & 1470 & 9.0 & 1463 & -12547 & -11496 & 0.864 \\
1865 & 1824 & 9.5 & 1816 & -15801 & -14522 & 0.862 \\
2123 & 2073 & 9.9 & 2063 & -18062 & -16665 & 0.863 \\
2491 & 2435 & 10.4 & 2424 & -21410 & -19837 & 0.862 \\
2779 & 2703 & 10.8 & 2688 & -23872 & -22223 & 0.862 \\
3151 & 3073 & 11.0 & 3060 & -27382 & -25443 & 0.862 \\
3589 & 3494 & 11.5 & 3478 & -31258 & -29182 & 0.859 \\
4093 & 3994 & 11.9 & 3977 & -35993 & -33751 & 0.860 \\
4585 & 4461 & 12.3 & 4435 & -40278 & -37849 & 0.860 \\
5233 & 5103 & 12.8 & 5075 & -46404 & -43647 & 0.861 \\
5775 & 5615 & 13.2 & 5591 & -51204 & -48313 & 0.859 \\
7011 & 6817 & 14.0 & 6781 & -62509 & -59292 & 0.857 \\
8589 & 8373 & 14.9 & 8328 & -77348 & -73600 & 0.858 \\
10185 & 9911 & 15.7 & 9863 & -92073 & -88023 & 0.857 \\
12215 & 11875 & 16.6 & 11756 & -110208 & -105587 & 0.856 \\
14363 & 13957 & 17.4 & 13856 & -130337 & -124983 & 0.854 \\
16757 & 16253 & 18.2 & 16148 & -152579 & -146697 & 0.854 \\
19381 & 18802 & 19.0 & 18678 & -176929 & -170500 & 0.853 \\
22231 & 21536 & 19.8 & 21386 & -203378 & -196263 & 0.853 \\
25315 & 24553 & 20.7 & 24357 & -232126 & -224329 & 0.853 \\
28897 & 27993 & 21.6 & 27750 & -264951 & -256802 & 0.852 \\
32565 & 31484 & 22.4 & 31209 & -298795 & -290163 & 0.852 \\
\hline
\end{tabular}




\section{Appendix B}

\section{Slip Length Simulations}

The data for measured slip lengths are provided in Table B.1. Slip lengths were calculated using a Couette flow set up. The velocity profiles of the liquid were spatially averaged along the height of the liquid in bins of 0.5 in height. These profiles were averaged for $6 \times 10^{5}$ time steps. The liquid was thermostatted every 100 time steps with $T^{*}=1 \pm 0.1$. The NVE integration was used.

Snap shots of simulations are provided in Figures B.1 and B.2.

Table B.1: Data for slip simulations. Slip Measurements are provided (reduced units) for Surfaces A-H and Runs 1-3. $l_{f}^{*}$ and $l_{c}^{*}$ are the lengths of the flat and curved portions of the surfaces.

\begin{tabular}{|l|l|l|rr|r|r|}
\hline Surface & $l_{f}^{*}$ (green) & $l_{c}^{*}$ (cyan) & Slip Length: & Run 1 & Run 2 & Run3 \\
\hline A & 60 & & & 33.9 & 52.6 & 72.7 \\
B & 60 & & 73.2 & 13.1 & 112.7 \\
C & 60 & & 10.8 & 3.5 & 30 \\
D & 30 & 30 & 56.4 & 24.3 & 100.5 \\
E & 30 & 34 & 13.7 & 11.8 & 23.8 \\
F & 30 & 38 & 10.6 & 10.3 & 19.5 \\
G & 30 & 42 & 8.2 & 8.6 & 14.4 \\
H & 30 & 46 & & 7.4 & 12.4 \\
\hline
\end{tabular}




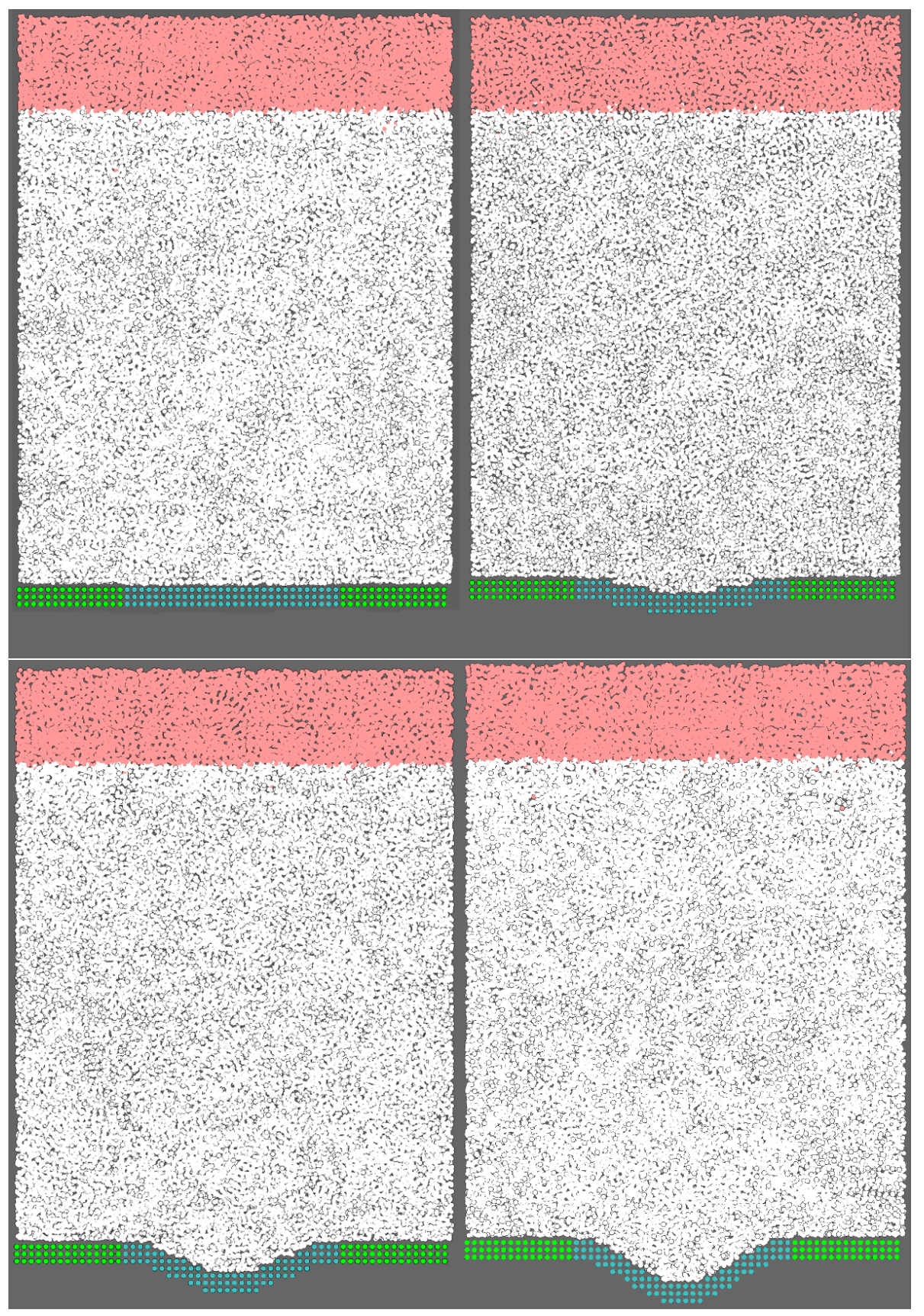

Figure B.1: Snap shots from slip simulations. The bottom and top right snap shots demonstrate how the steps in the curved region affect the liquid as liquid atoms in these regions appear to bunch up in the steps. This bunching up supports the value of 2 for the scaled arc lengths (as opposed to $\sqrt{1}$, the hypotenuse). 


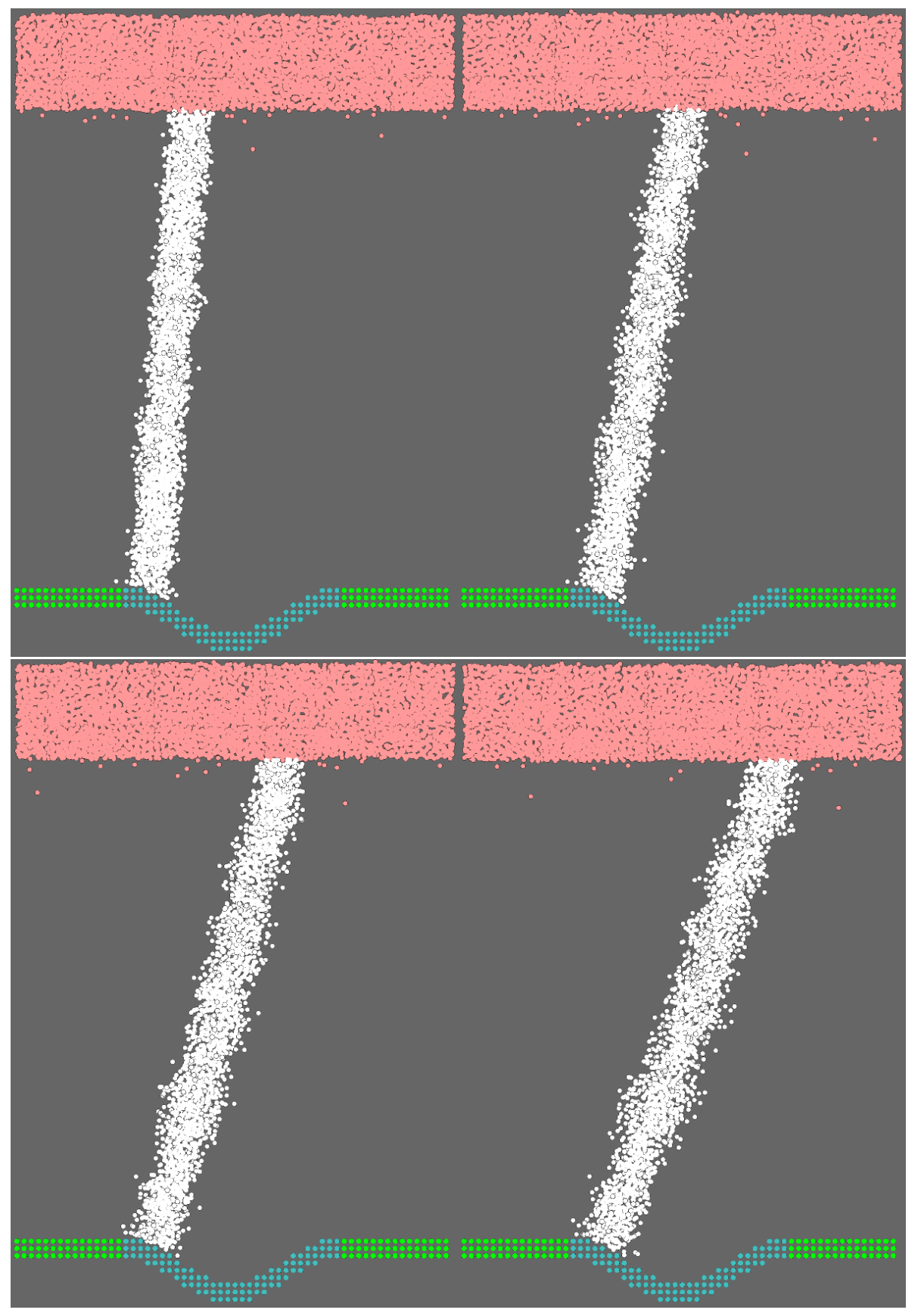

Figure B.2: Flow of slip simulations. A snapshot of one simulation showing a smooth transition from the low slip region to the high slip region. 



\section{Appendix C}

\section{Drop Simulations}

\section{C.1 Measuring Contact Size}

To measure the contact size, we fitted an elliptical profile along the surface of the drop. Figure C.1 shows the radial distances from the center of mass in the xz-plane (x-axis) plotted along the height of the drop (y-axis). We consider atoms below the height of the center of mass (blue), and take spacial averages along the height of the drop to estimate the edge of the drop (light blue). An ellipse is fitted to the data (red) and the contact size is where the drop height is zero.

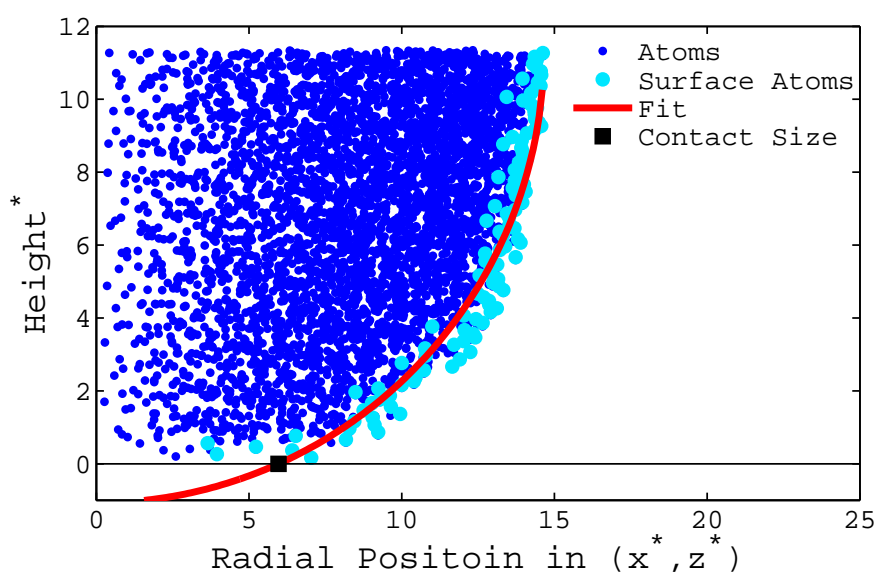

Figure C.1: Measuring contact size. The geometry of the drop is found using an elliptical fit (red), and this geometry determines the contact size (ם).

\section{C.2 Results}

We provide a summary of all data in Table C.1. Figures C.2 to C.7 provide the velocity profile analyses for each set of simulations. The averaged and the 
Appendix C

instantaneous drop center of mass velocities as a function of time are presented in Figures C.8 to C.13. 


\begin{tabular}{|c|c|c|c|c|c|c|c|c|c|c|c|}
\hline Sim & $R^{*}$ & $b^{*}$ & $f_{y}^{*}$ & $\kappa^{-1 *}$ & $l^{*}$ & $h^{*}$ & $h_{c m}^{*}$ & $\alpha$ & $U_{r}^{*} / \alpha$ & $U_{s}^{*} / \alpha$ & $U_{c m}^{*} / \alpha$ \\
\hline 1 & 10.0 & 38 & $3 e-3$ & 25 & 3.6 & 16.0 & 9.2 & 0.17 & 0.793 & 0.580 & $1.362 \pm 0.056$ \\
\hline 1 & 13.3 & 38 & $3 e-3$ & 25 & 5.9 & 20.5 & 11.0 & 0.17 & 0.620 & 0.690 & $1.282 \pm 0.031$ \\
\hline 1 & 16.7 & 38 & $3 e-3$ & 25 & 9.8 & 23.3 & 12.3 & 0.17 & 0.519 & 0.744 & $1.230 \pm 0.025$ \\
\hline 1 & 20.0 & 38 & $3 e-3$ & 25 & 13.8 & 25.9 & 13.3 & 0.17 & 0.478 & 0.763 & $1.211 \pm 0.017$ \\
\hline 1 & 23.3 & 38 & $3 e-3$ & 25 & 18.3 & 28.1 & 14.1 & 0.17 & 0.447 & 0.770 & $1.188 \pm 0.015$ \\
\hline 1 & 26.7 & 38 & $3 e-3$ & 25 & 22.9 & 30.3 & 14.7 & 0.17 & 0.420 & 0.771 & $1.176 \pm 0.011$ \\
\hline 2 & 10.0 & 48 & $3 e-3$ & 25 & 3.6 & 16.0 & 9.3 & 0.08 & 0.794 & 0.751 & $1.532 \pm 0.085$ \\
\hline 2 & 13.3 & 48 & $3 e-3$ & 25 & 5.9 & 20.5 & 11.0 & 0.08 & 0.602 & 0.892 & $1.486 \pm 0.066$ \\
\hline 2 & 16.7 & 48 & $3 e-3$ & 25 & 9.8 & 23.3 & 12.4 & 0.08 & 0.534 & 0.913 & $1.417 \pm 0.045$ \\
\hline 2 & 20.0 & 48 & $3 e-3$ & 25 & 13.8 & 25.9 & 13.4 & 0.08 & 0.456 & 0.936 & $1.370 \pm 0.030$ \\
\hline 3 & 10.0 & 38 & $3 e-3$ & 25 & 3.6 & 16.0 & 9.2 & 0.08 & 0.675 & 0.561 & $1.254 \pm 0.097$ \\
\hline 3 & 13.3 & 38 & $3 e-3$ & 25 & 5.9 & 20.5 & 11.1 & 0.08 & 0.577 & 0.691 & $1.241 \pm 0.085$ \\
\hline 3 & 16.7 & 38 & $3 e-3$ & 25 & 9.8 & 23.3 & 12.3 & 0.08 & 0.501 & 0.686 & $1.162 \pm 0.048$ \\
\hline 3 & 20.0 & 38 & $3 e-3$ & 25 & 13.8 & 25.9 & 13.3 & 0.08 & 0.444 & 0.743 & $1.160 \pm 0.036$ \\
\hline 4 & 10.0 & 48 & $3 e-3$ & 25 & 3.6 & 16.0 & 10.0 & 0.03 & 0.825 & 0.850 & $1.740 \pm 0.232$ \\
\hline 4 & 13.3 & 48 & $3 e-3$ & 25 & 5.9 & 20.5 & 11.7 & 0.03 & 0.614 & 0.831 & $1.456 \pm 0.191$ \\
\hline 4 & 16.7 & 48 & $3 e-3$ & 25 & 9.8 & 23.3 & 12.7 & 0.03 & 0.492 & 0.877 & $1.356 \pm 0.094$ \\
\hline 4 & 20.0 & 48 & $3 e-3$ & 25 & 13.8 & 25.9 & 13.9 & 0.03 & 0.429 & 0.897 & $1.331 \pm 0.088$ \\
\hline 4 & 23.3 & 48 & $3 e-3$ & 25 & 18.3 & 28.1 & 14.4 & 0.03 & 0.420 & 0.945 & $1.350 \pm 0.063$ \\
\hline 4 & 26.7 & 48 & $3 e-3$ & 25 & 22.9 & 30.3 & 14.9 & 0.03 & 0.419 & 0.866 & $1.266 \pm 0.060$ \\
\hline 5 & 13.3 & 115 & $3 e-4$ & 79 & 2.2 & 24.3 & 13.5 & 0.12 & 0.470 & 0.263 & $0.761 \pm 0.053$ \\
\hline 5 & 16.7 & 115 & $3 e-4$ & 79 & 3.5 & 29.7 & 16.2 & 0.12 & 0.476 & 0.316 & $0.805 \pm 0.030$ \\
\hline 5 & 20.0 & 115 & $3 e-4$ & 79 & 5.0 & 34.8 & 20.6 & 0.12 & 0.526 & 0.336 & $0.906 \pm 0.025$ \\
\hline 5 & 23.3 & 115 & $3 e-4$ & 79 & 6.9 & 39.5 & 21.9 & 0.12 & 0.519 & 0.388 & $0.908 \pm 0.020$ \\
\hline 5 & 26.7 & 115 & $3 e-4$ & 79 & 9.0 & 44.3 & 24.0 & 0.12 & 0.483 & 0.445 & $0.918 \pm 0.018$ \\
\hline 5 & 30.0 & 115 & $3 e-4$ & 79 & 11.4 & 48.4 & 26.1 & 0.12 & 0.474 & 0.470 & $0.926 \pm 0.017$ \\
\hline 6 & 13.3 & 158 & $3 e-4$ & 79 & 2.2 & 24.3 & 14.0 & 0.08 & 0.487 & 0.291 & $0.816 \pm 0.049$ \\
\hline 6 & 16.7 & 158 & $3 e-4$ & 79 & 3.5 & 29.7 & 16.7 & 0.08 & 0.522 & 0.462 & $0.992 \pm 0.039$ \\
\hline 6 & 20.0 & 158 & $3 e-4$ & 79 & 5.0 & 34.8 & 19.2 & 0.08 & 0.550 & 0.532 & $1.088 \pm 0.030$ \\
\hline 6 & 23.3 & 158 & $3 e-4$ & 79 & 6.9 & 39.5 & 21.7 & 0.08 & 0.555 & 0.557 & $1.111 \pm 0.019$ \\
\hline 6 & 26.7 & 158 & $3 e-4$ & 79 & 9.0 & 44.3 & 24.0 & 0.08 & 0.510 & 0.605 & $1.110 \pm 0.016$ \\
\hline 6 & 30.0 & 158 & $3 e-4$ & 79 & 11.4 & 48.4 & 26.2 & 0.08 & 0.521 & 0.633 & $1.140 \pm 0.014$ \\
\hline
\end{tabular}

Table C.1: Drop results. Radius $R^{*}$, slip length $b^{*}$, gravity term $f_{y}^{*}$, capillary length $\kappa^{-1^{*}}$, contact size $l^{*}$, drop height $h^{*}$, height of center of mass $h_{c m}^{*}$, angle of inclination $\alpha$ (radians), scaled rolling velocity $U_{r}^{*} / \alpha$, scaled slip velocity $U_{s}^{*} / \alpha$, and scaled center of mass velocity $U_{c m}^{*} / \alpha$ with error of 1 standard deviation from measured velocities. 


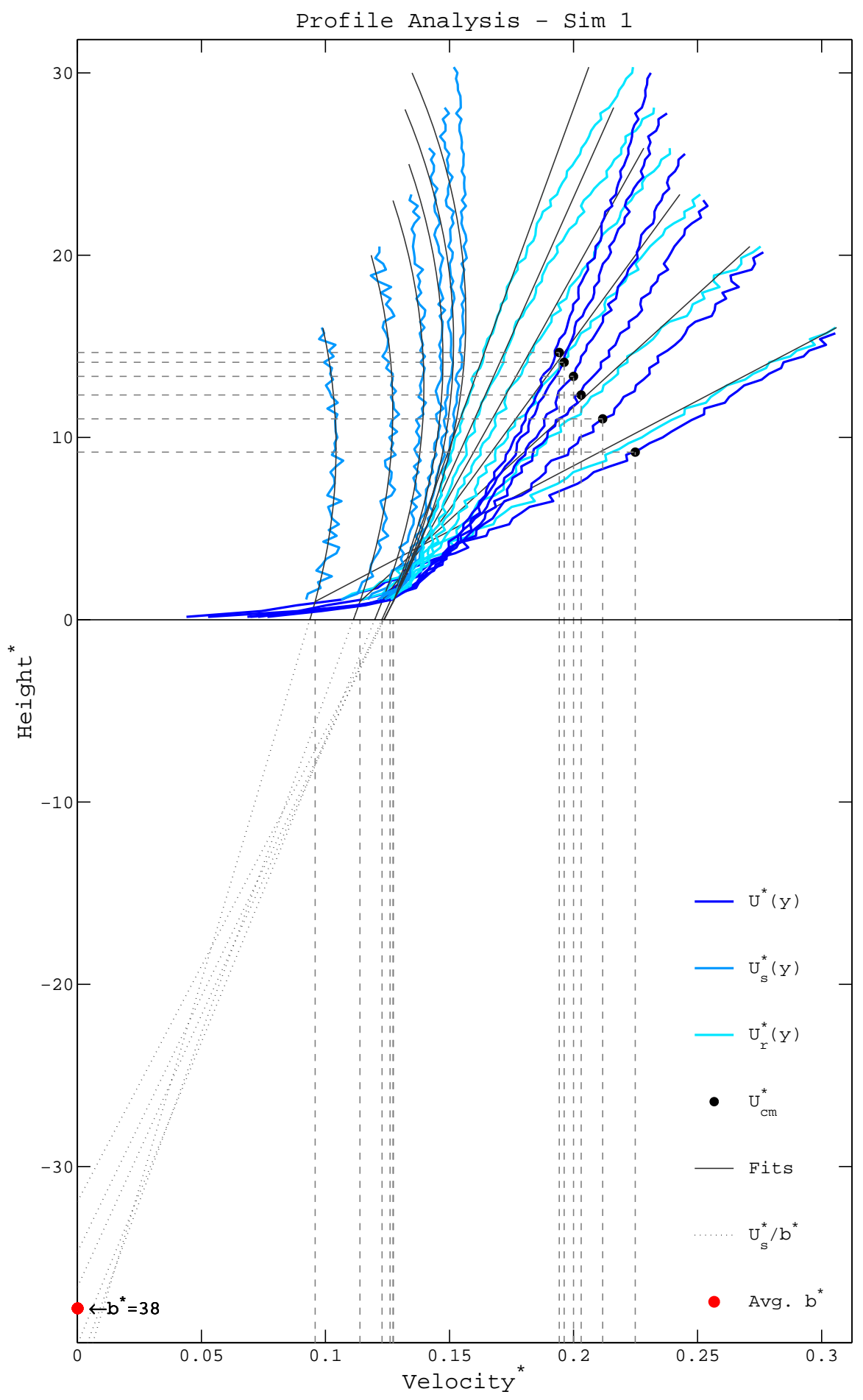

Figure C.2: Profile analysis - Simulation 1 $g^{*}=3 \times 10^{-3}, \alpha=9.5^{\circ}, \kappa^{-1 *}=25, b^{*}=38$ 


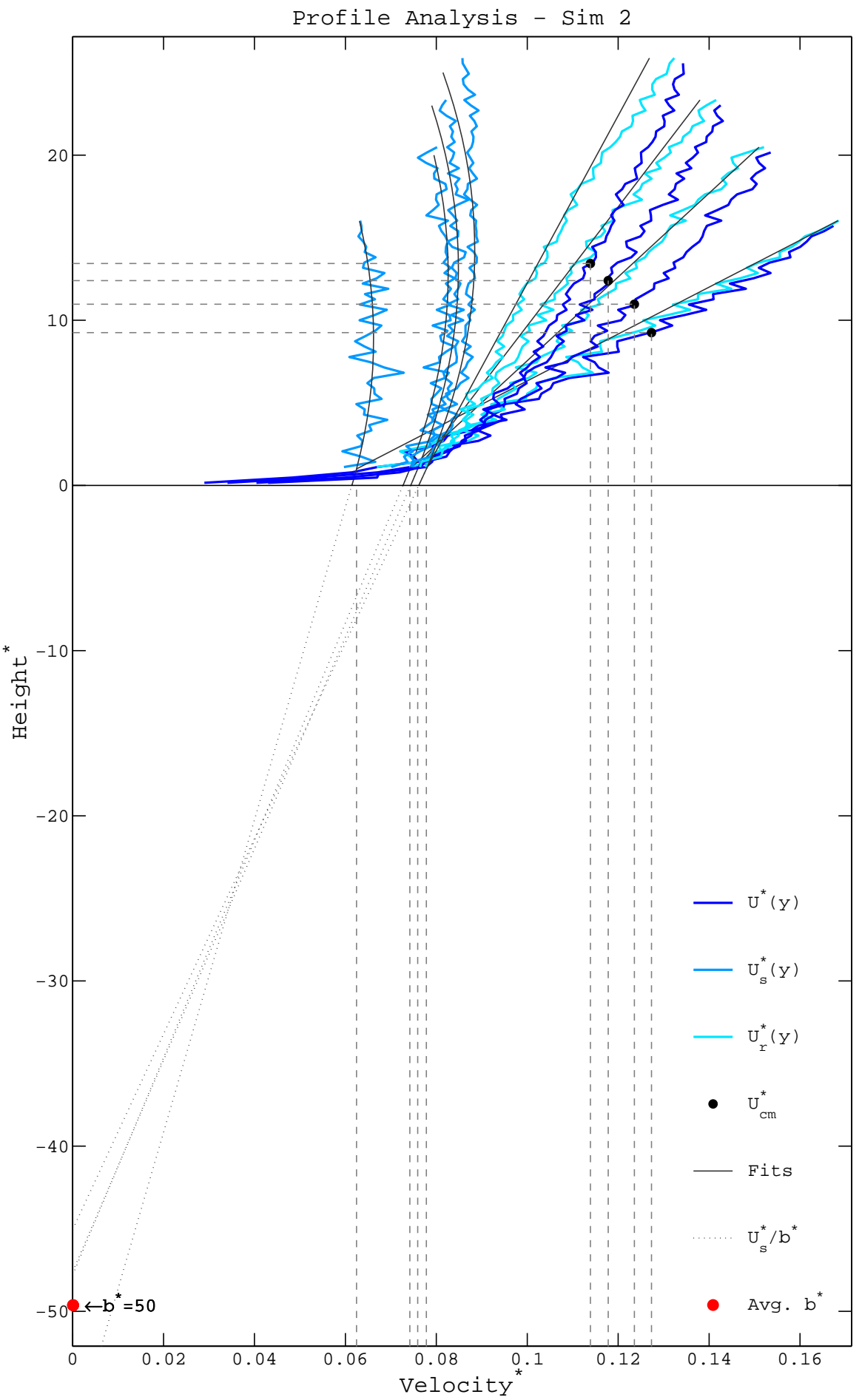

Figure C.3: Profile analysis - Simulation 2 $g^{*}=3 \times 10^{-3}, \alpha=4.8^{\circ}, \kappa^{-1 *}=25, b^{*}=48$ 


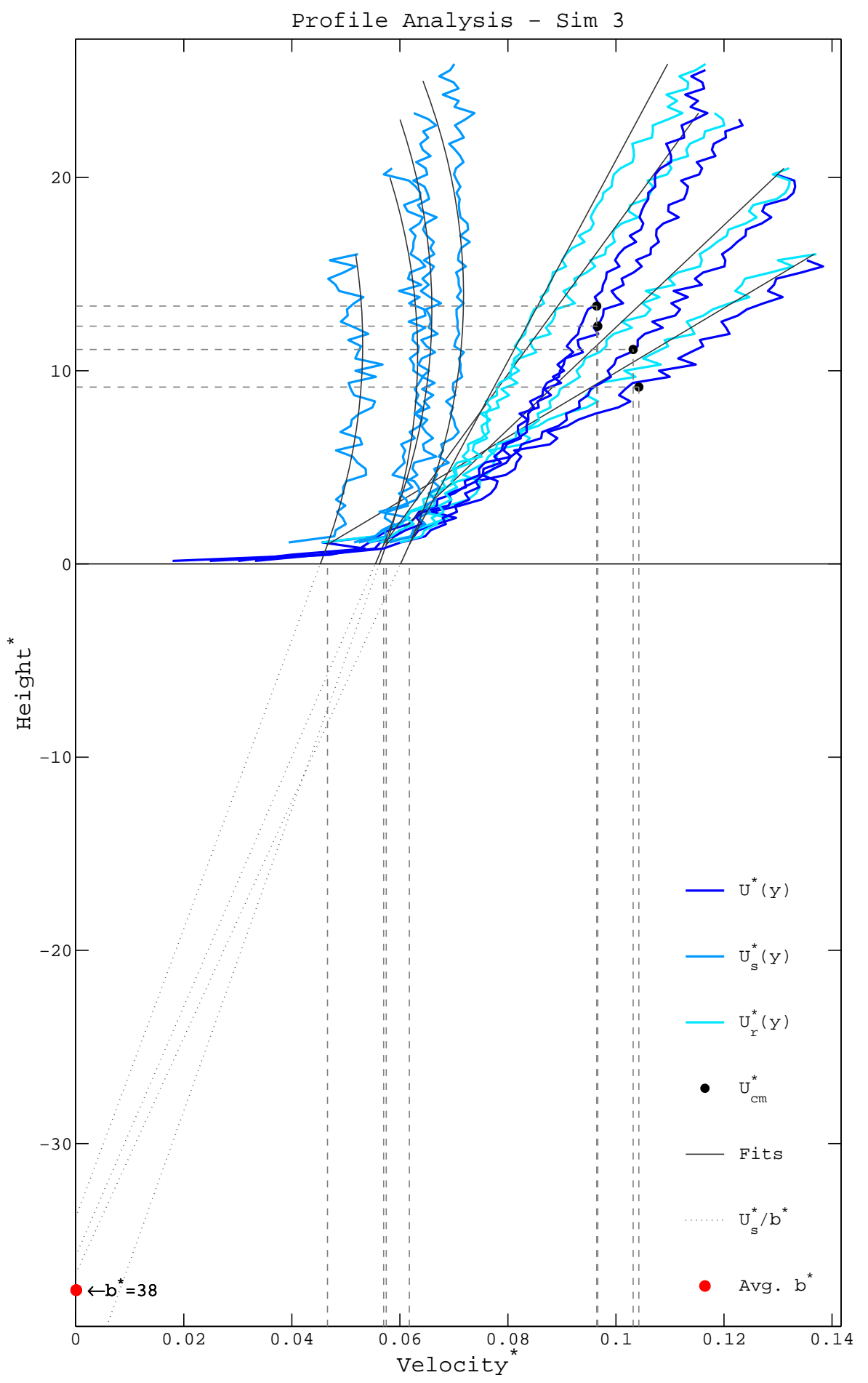

Figure C.4: Profile analysis - Simulation 3 $g^{*}=3 \times 10^{-3}, \alpha=4.8^{\circ}, \kappa^{-1 *}=25, b^{*}=38$ 


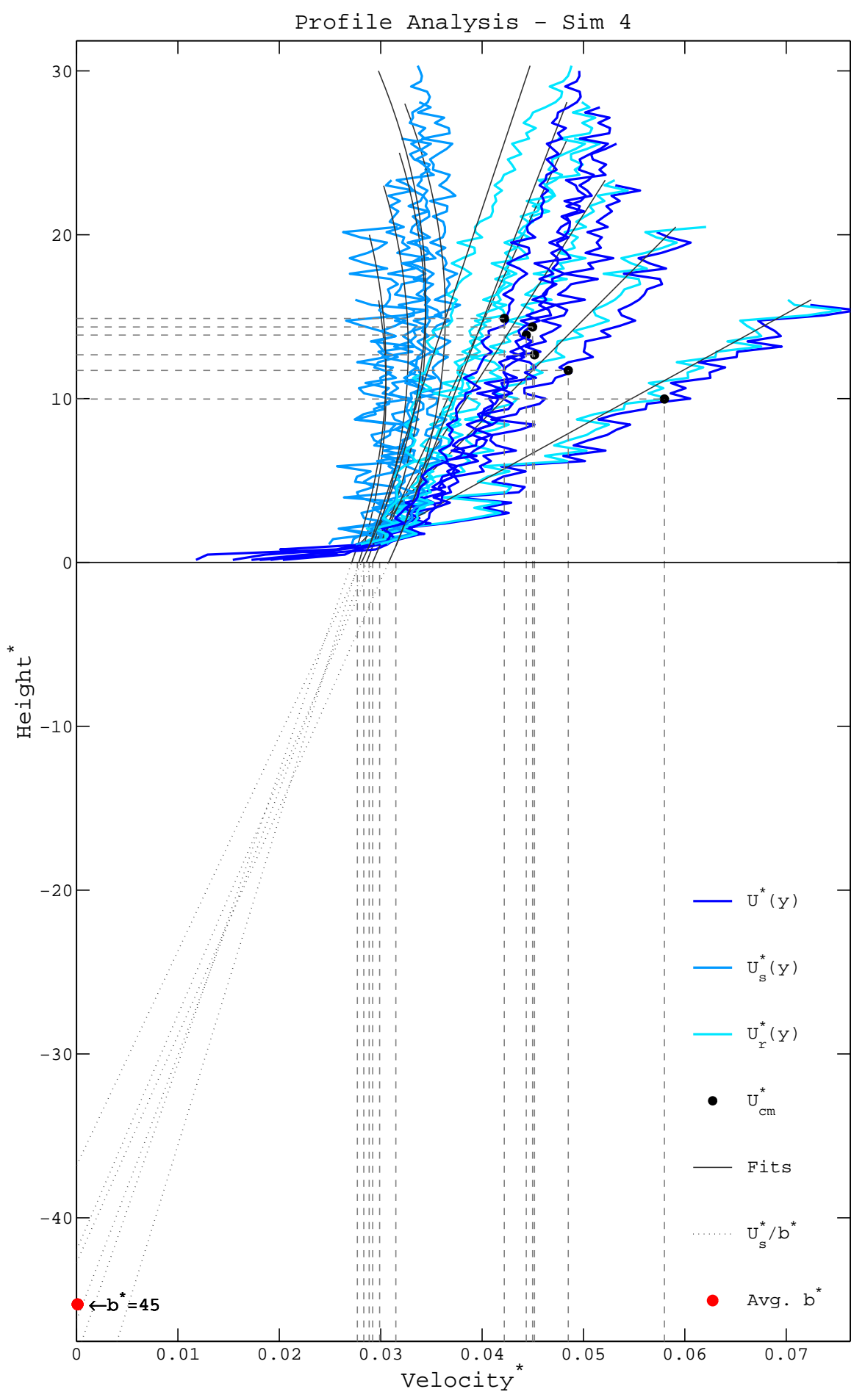

Figure C.5: Profile analysis - Simulation 4 $g^{*}=3 \times 10^{-3}, \alpha=1.9^{\circ}, \kappa^{-1 *}=25, b^{*}=48$ 


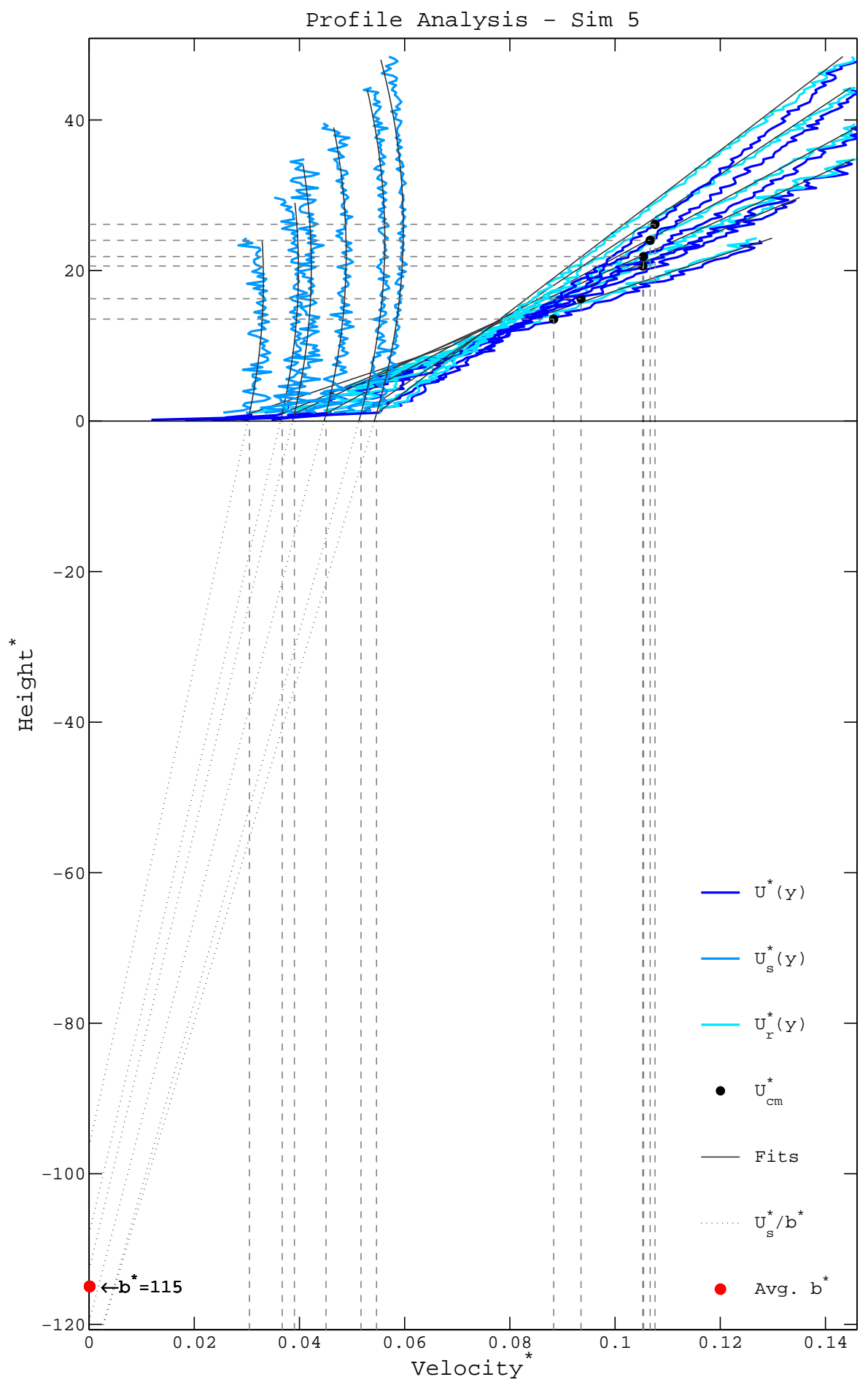

Figure C.6: Profile analysis - Simulation 5 $g^{*}=3 \times 10^{-4}, \alpha=6.7^{\circ}, \kappa^{-1 *}=79, b^{*}=115$ 


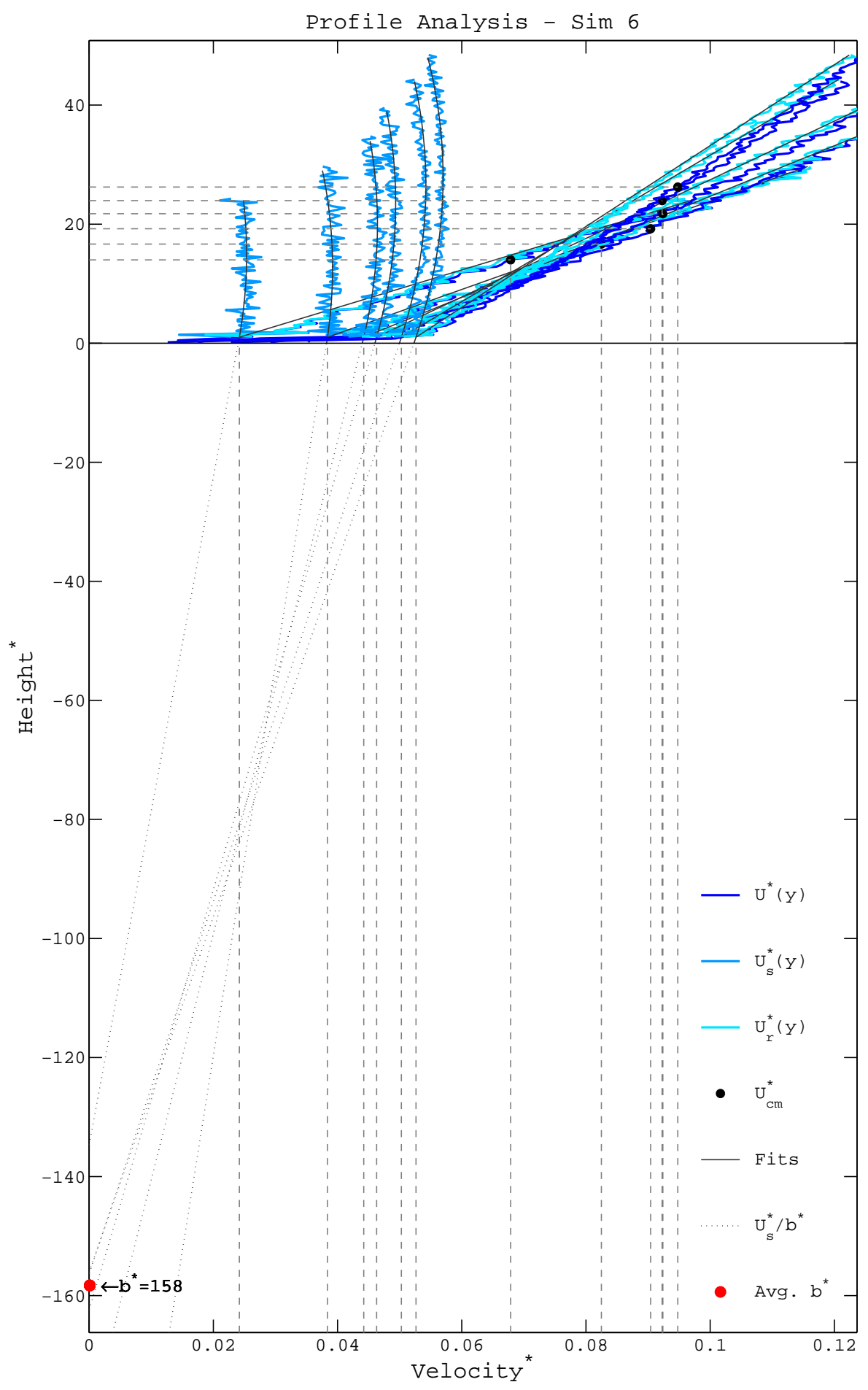

Figure C.7: Profile analysis - Simulation 6 $g^{*}=3 \times 10^{-4}, \alpha=4.8^{\circ}, \kappa^{-1 *}=79, b^{*}=158$ 

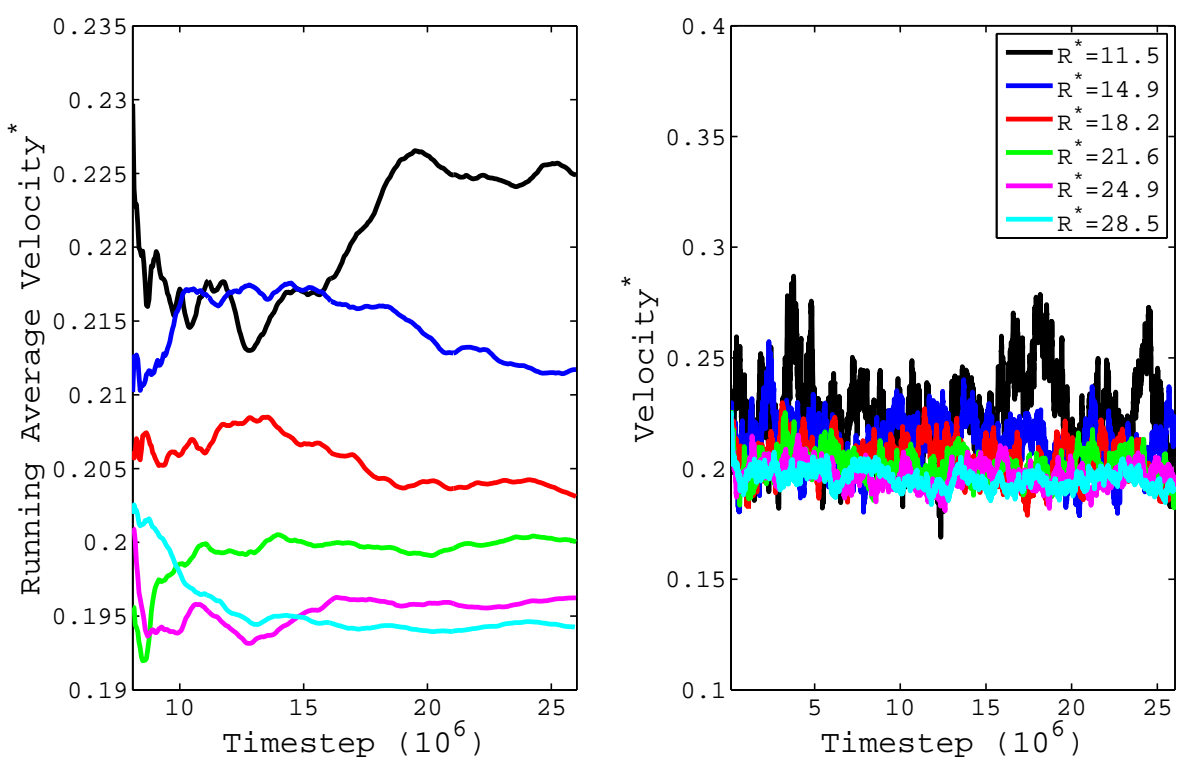

Figure C.8: Data - Simulation 1

$g^{*}=3 \times 10^{-3}, \alpha=9.5^{\circ}, \kappa^{-1 *}=22, b^{*}=36$
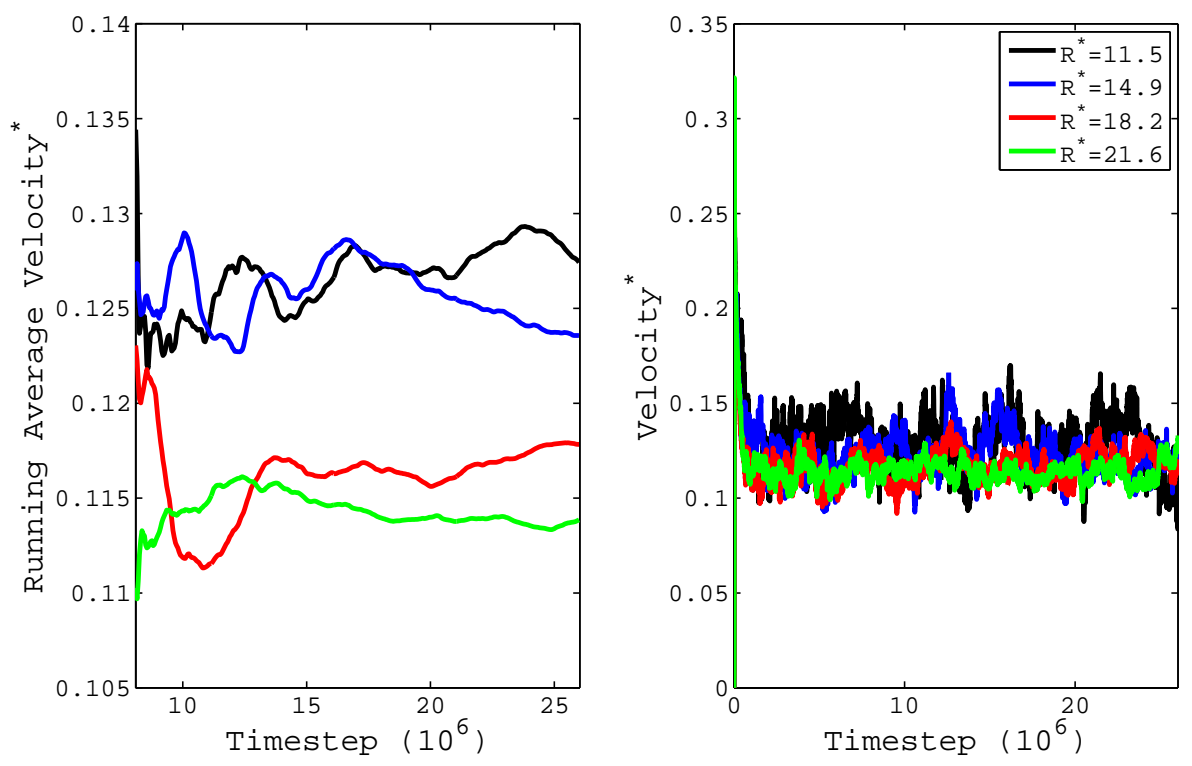

Figure C.9: Data - Simulation 2

$g^{*}=3 \times 10^{-3}, \alpha=4.8^{\circ}, \kappa^{-1 *}=22, b^{*}=49$ 

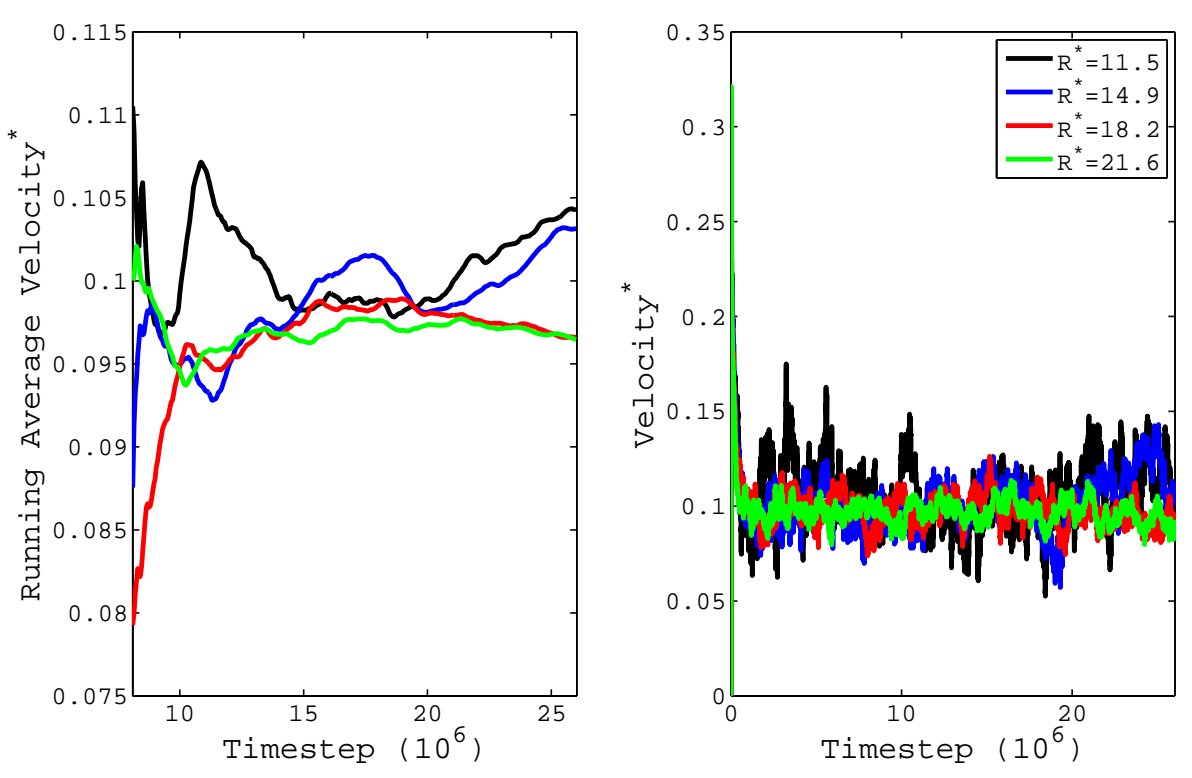

Figure C.10: Data - Simulation 3

$g^{*}=3 \times 10^{-3}, \alpha=4.8^{\circ}, \kappa^{-1 *}=22, b^{*}=36$
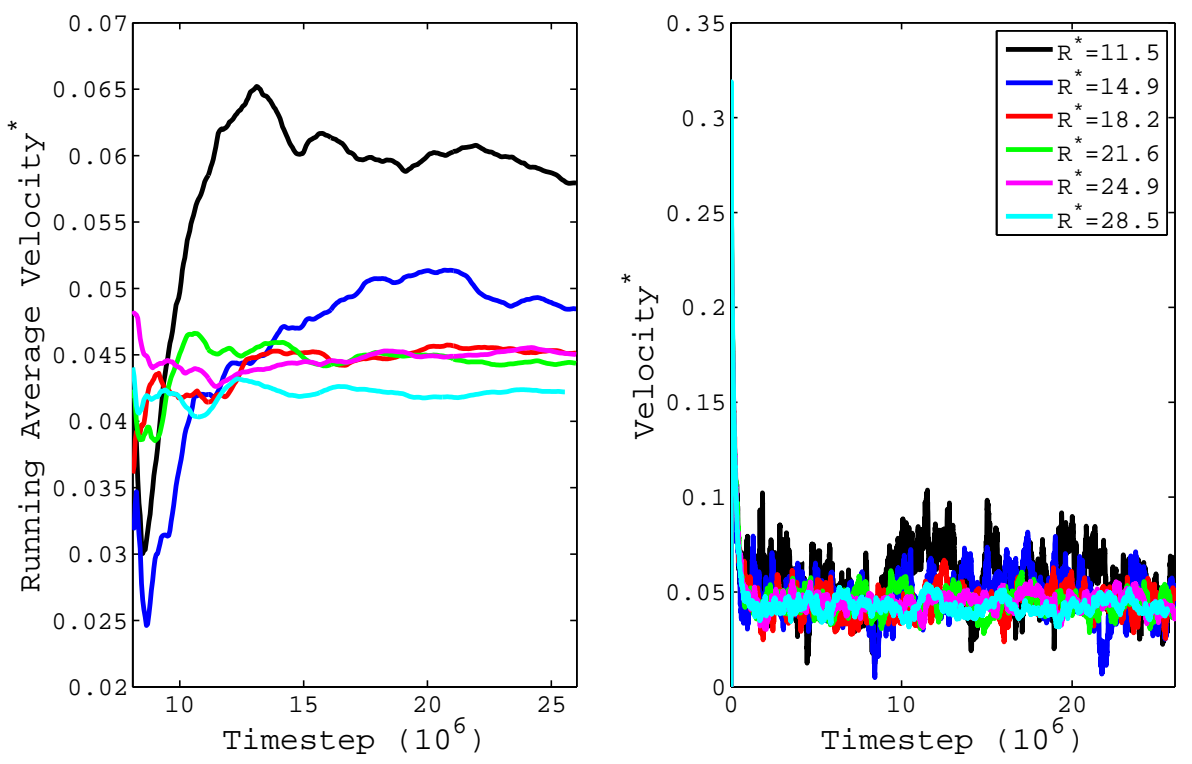

Figure C.11: Data - Simulation 4

$g^{*}=3 \times 10^{-3}, \alpha=1.9^{\circ}, \kappa^{-1 *}=22, b^{*}=49$ 

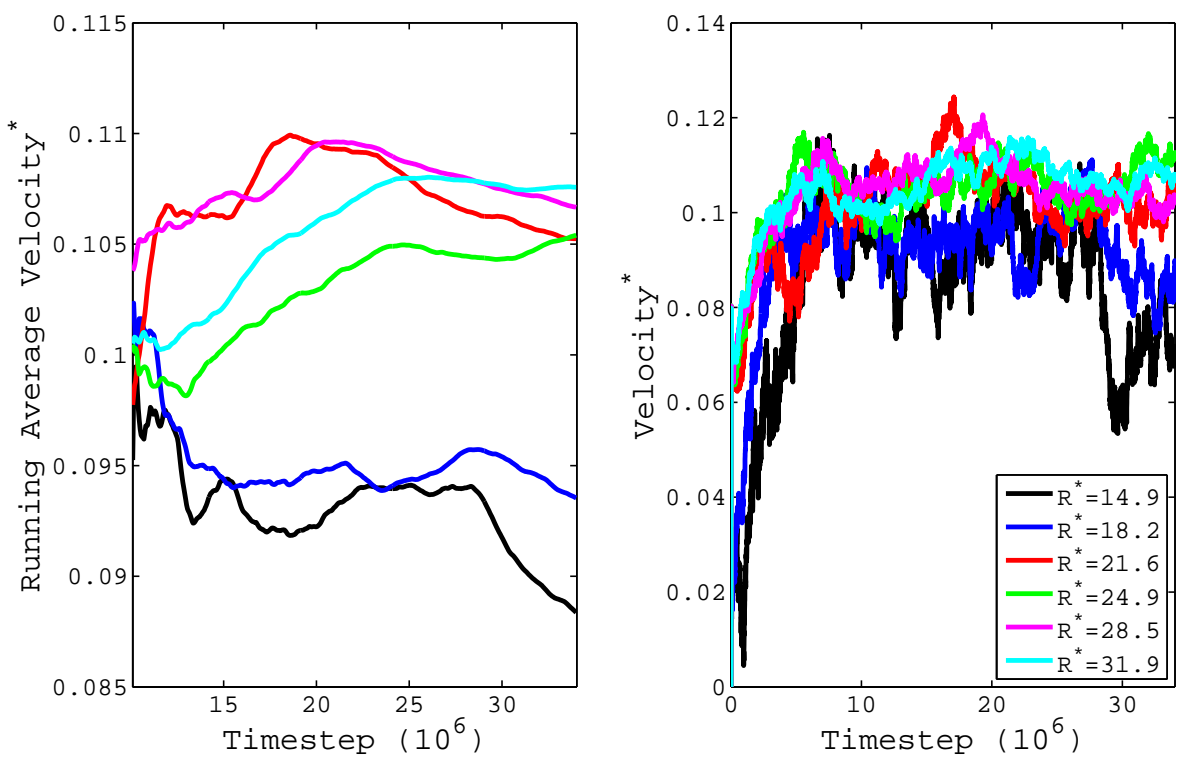

Figure C.12: Data - Simulation 5

$g^{*}=3 \times 10^{-4}, \alpha=6.7^{\circ}, \kappa^{-1 *}=70, b^{*}=92$
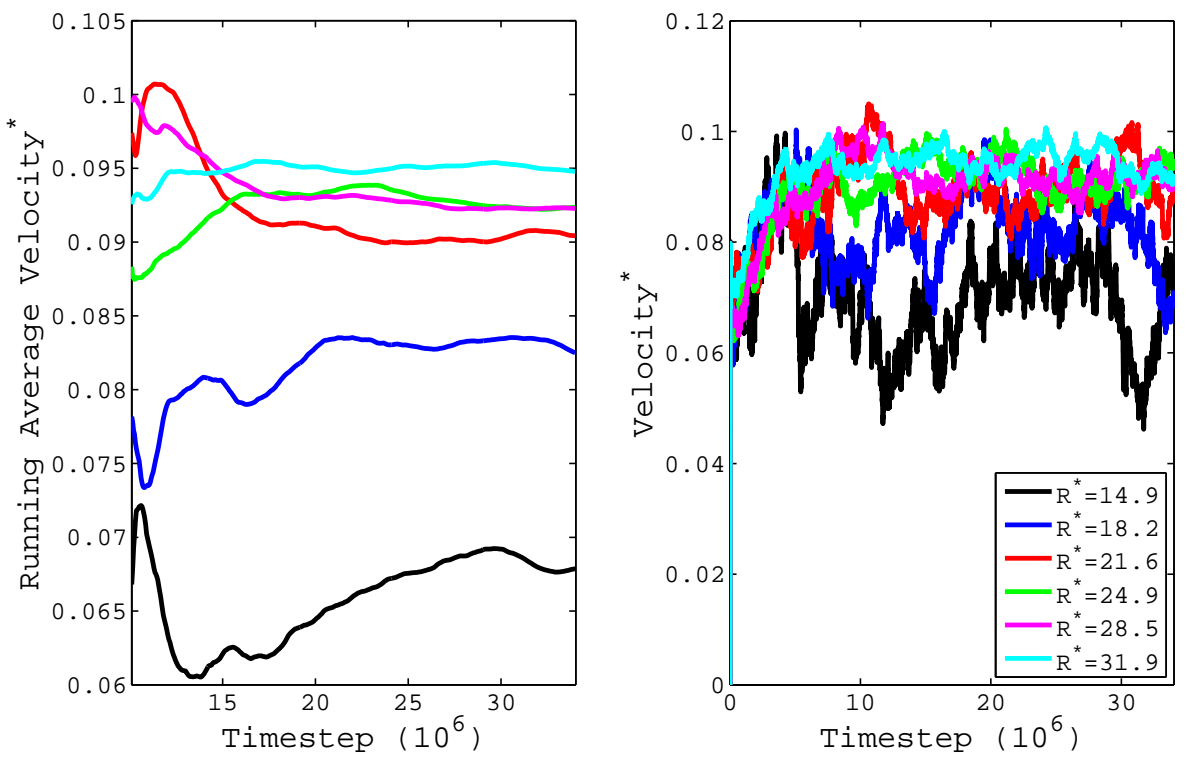

Figure C.13: Data - Simulation 6

$g^{*}=3 \times 10^{-4}, \alpha=4.8^{\circ}, \kappa^{-1 *}=70, b^{*}=118$ 


\section{Bibliography}

[1] J. Barrat and L. Bocquet, "Large slip effect at a nonwetting fluid-solid interface," Physical Review Letters, vol. 82, no. 23, pp. 4671-4674, 1999. Effects of pressure on slip length.

[2] W. Barthlott and C. Neinhuis, "Purity of the sacred lotus, or escape from contamination in biological surfaces," Planta, vol. 202, no. 1, pp. 1-8, 1997.

[3] K. S. Novoselov, A. K. Geim, S. V. Morozov, D. Jiang, Y. Zhang, S. V. Dubonos, I. V. Grigorieva, and A. A. Firsov, "Electric field effect in atomically thin carbon films," Science, vol. 306, no. 5696, pp. 666-669, 2004.

[4] F. Ercolessi, "A molecular dynamics primer," pp. 1-52, Mar 1997.

[5] L. Verlet, "Computer "experiments" on classical fluids. i. thermodynamical properties of lennard-jones molecules," Phys. Rev., vol. 159, pp. 98-103, Jul 1967.

[6] J. v Neumann, "Proof of the quasi-ergodic hypothesis," Proceedings of the National Academy of Sciences of the United States of America, vol. 18, p. 70, Jan 1932.

[7] S. Plimpton, "Fast parallel algorithms for short-range molecular dynamics," Journal of Computational Physics, vol. 117, no. 1, pp. 1-19, 1995.

[8] W. Humphrey, A. Dalke, and K. Schulten, "Vmd - visual molecular dynamics," Journal of Molecular Graphics, vol. 14, pp. 33-38, 1996.

[9] B. Smit, "Phase diagrams of lennard-jones fluids," The Journal of Chemical Physics, vol. 96, no. 11, p. 8639, 1992.

[10] V. Baidakov, G. Chernykh, and S. Protsenko, "Effect of the cut-off radius of the intermolecular potential on phase equilibrium and surface tension in lennard-jones systems," Chemical Physics Letters, Jan 2000. 
[11] M. J. P. Nijmeijer, C. Bruin, A. van Woerkom, A. Bakker, and J. V. Leeuwen, "Molecular dynamics of the surface tension of a drop," Journal of Chemical Physics, vol. 96, Jan 1992.

[12] J. W. Lee and G. D. Stein, "Structure change with size of argon clusters formed in laval nozzle beams," Journal of Physical Chemistry, vol. 91, pp. 2450-2457, May 1987.

[13] J. J. Potoff and A. Z. Panagiotopoulos, "Surface tension of the threedimensional lennard-jones fluid from histogram-reweighting monte carlo simulations," The Journal of Chemical Physics, vol. 112, p. 6411, Jan 2000.

[14] D. Stansfield, "The surface tensions of liquid argon and nitrogen," Proceedings of the Physical Society, vol. 72, p. 854, Nov 1958.

[15] B. Hess, "Determining the shear viscosity of model liquids from molecular dynamics simulations," The Journal of Chemical Physics, vol. 116, p. 209, 2002.

[16] D. Schebarchov and S. Hendy, "Dynamics of capillary absorption of droplets by carbon nanotubes," Physical Review E, vol. 78, p. 046309, Oct 2008.

[17] K. Tankeshwar, K. Pathak, and S. Ranganathan, "The shear viscosity of lennard-jones fluids," Journal of Physics C: Solid State Physics, vol. 21, pp. 3607-3617, 1988.

[18] R. Rowley and M. Painter, "Diffusion and viscosity equations of state for a lennard-jones fluid obtained from molecular dynamics simulations," International Journal of Thermophysics, vol. 18, no. 5, pp. 1109-1121, 1997. Viscosity values.

[19] L. Woodcock, "Equation of state for the viscosity of lennard-jones fluids," American Institute of Chemical Engineers, vol. 52, no. 2, pp. 438-446, 2005.

[20] N. J. Lund, P. Zhang, K. K. Mahelona, and S. C. Hendy, "Slippery issues," In preparation, Oct 2011.

[21] E. Lauga, M. Brenner, and H. Stone, "Microfluidics: the no-slip boundary condition," Arxiv preprint cond-mat/0501557, 2005.

[22] D. Richard and D. Quéré, "Viscous drops rolling on a tilted non-wettable solid," EPL (Europhysics Letters), vol. 48, pp. 286-291, 1999. 
[23] D. Quéré, "Non-sticking drops," Reports on Progress in Physics, vol. 68, p. 2495, 2005.

[24] J. Servantie and M. Müller, "Statics and dynamics of a cylindrical droplet under an external body force," The Journal of Chemical Physics, vol. 128, p. 014709, 2008.

[25] B. Mognetti, H. Kusumaatmaja, and J. Yeomans, "Drop dynamics on hydrophobic and superhydrophobic surfaces," Faraday Discussions, vol. 146, pp. 153-165, 2010.

[26] M. Hunter, S. C. Hendy, K. K. Mahelona, R. G. Stanley, and G. R. Willmott, "Nuclear magnetic resonance investigation of rolling drops on superhydrophobic surfaces," Fifth International Conference on Advanced Materials and Nanotechnology, Feb 2011.

[27] T. Blake, "Kinetics of liquid/liquid displacement," Journal of Colloid and Interface Science, vol. 30, pp. 421-423, Jul 1969.

[28] T. D. Blake, A. Clarke, J. D. Coninck, and M. J. D. Ruijter, "Contact angle relaxation during droplet spreading: Comparison between molecular kinetic theory and molecular dynamics," Langmuir, vol. 13, pp. 2164-2166, Apr 1997.

[29] T. Blake and J. D. Coninck, "The influence of solid-liquid interactions on dynamic wetting," Advances in colloid and interface science, vol. 96, no. 1-3, pp. 21-36, 2002.

[30] L. Mahadevan and Y. Pomeau, "Rolling droplets," Physics of fluids, vol. 11, p. 2449, 1999.

[31] H. Kim, "Sliding of liquid drops down an inclined solid surface," Journal of Colloid and Interface Science, vol. 247, pp. 372-380, Mar 2002.

[32] N. L. Grand, A. Daerr, and L. Limat, "Shape and motion of drops sliding down an inclined plane," Journal of Fluid Mechanics, vol. 541, pp. 293-315, Jan 2005.

[33] M. J. D. Ruijter, T. D. Blake, and J. D. Coninck, "Dynamic wetting studied by molecular modeling simulations of droplet spreading," Langmuir, vol. 15, pp. 7836-7847, Oct 1999. 
[34] D. Brenner, O. Shenderova, J. Harrison, S. Stuart, B. Ni, and S. Sinnott, "A second-generation reactive empirical bond order (rebo) potential energy expression for hydrocarbons," Journal of Physics: Condensed Matter, vol. 14, p. 783 , Feb 2002.

[35] S. J. Stuart, A. B. Tutein, and J. A. Harrison, "A reactive potential for hydrocarbons with intermolecular interactions," The Journal of Chemical Physics, vol. 112, p. 6472, Jan 2000.

[36] M. S. Daw and M. Baskes, "Semiempirical, quantum mechanical calculation of hydrogen embrittlement in metals," Physical Review Letters, vol. 50, pp. 1285-1288, Apr 1983.

[37] W. Xiao, M. Baskes, and K. Cho, "Meam study of carbon atom interaction with ni nano particle," Surface Science, vol. 603, no. 13, pp. 1985-1998, 2009.

[38] J. Uddin, M. Baskes, S. Srinivasan, and T. Cundari, "Modified embedded atom method study of the mechanical properties of carbon nanotube reinforced nickel composites," Physical Review B, vol. 81, p. 104103, Jan 2010.

[39] W. Goddard, B. Merinov, A. V. Duin, T. Jacob, M. Blanco, V. Molinero, S. Jang, and Y. Jang, "Multi-paradigm multi-scale simulations for fuel cell catalysts and membranes," Molecular Simulation, vol. 32, no. 3, pp. 251-268, 2006.

[40] K. Chenoweth, A. V. Duin, and W. G. Iii, "Reaxff reactive force field for molecular dynamics simulations of hydrocarbon oxidation," The Journal of Physical Chemistry A, vol. 112, no. 5, pp. 1040-1053, 2008.

[41] C. Sanz-Navarro, P. Astrand, D. Chen, M. Rønning, A. C. T. van Duin, T. Jacob, and I. W. A. Goddard, "Molecular dynamics simulations of the interactions between platinum clusters and carbon platelets," The Journal of Physical Chemistry C, vol. 112, pp. 1392-1402, Jan 2008.

[42] C. F. Sanz-Navarro, P.-O. Åstrand, D. Chen, M. Rønning, A. C. T. van Duin, J. E. Mueller, and W. A. G. Iii, "Molecular dynamics simulations of carbon-supported ni clusters using the reax reactive force field," The Journal of Physical Chemistry C, vol. 112, pp. 12663-12668, Jul 2008. 
[43] S. Huang, D. Mainardi, and P. Balbuena, "Structure and dynamics of graphite-supported bimetallic nanoclusters," Surface Science, vol. 545, no. 3, pp. 163-179, 2003.

[44] H. Li, X. Ren, and X. Guo, "Monte carlo studies on the filling process of carbon nanotubes with nickel," Chemical Physics Letters, vol. 437, no. 1-3, pp. 108-111, 2007.

[45] Y. Hernandez, V. Nicolosi, M. Lotya, F. M. Blighe, Z. Sun, S. De, I. T. Mcgovern, B. Holland, M. Byrne, Y. K. Gun'ko, J. J. Boland, P. Niraj, G. Duesberg, S. Krishnamurthy, R. Goodhue, J. Hutchison, V. Scardaci, A. C. Ferrari, and J. N. Coleman, "High-yield production of graphene by liquid-phase exfoliation of graphite," Nature Nanotechnology, vol. 3, p. 563, Aug 2008.

[46] Z. Liu, J. Liu, Y. Cheng, and Q. Zheng, "Interlayer binding energy of graphite-a direct experimental determination," Arxiv preprint arXiv:1104.1469, 2011.

[47] A. P. LaGrow, S. Cheong, B. Ingham, M. F. Toney, and R. D. Tilley, "Shape control of nickel nanoparticles," Fifth International Conference on Advanced Materials and Nanotechnology, pp. 1-1, Feb 2010.

[48] S. Carenco, C. Boissière, L. Nicole, C. Sanchez, P. L. Floch, and N. Mézailles, "Controlled design of size-tunable monodisperse nickel nanoparticles," Chemistry of Materials, vol. 22, pp. 1340-1349, Jan 2010.

[49] G. Bertoni, L. Calmels, A. Altibelli, and V. Serin, "First-principles calculation of the electronic structure and eels spectra at the graphene/ni(111) interface," Physical Review B, vol. 71, p. 075402, Feb 2005.

[50] M. Fuentes-Cabrera, M. Baskes, and A. Melechko, "Bridge structure for the graphene/ni (111) system: A first principles study," Physical Review B, Jan 2008.

[51] J. Lahiri, T. S. Miller, A. J. Ross, L. Adamska, I. Oleynik, and M. Batzill, "Graphene growth and stability at nickel surfaces," New Journal of Physics, vol. 13, p. 025001, Feb 2011.

[52] L. A. Girifalco and R. A. Lad, "Energy of cohesion, compressibility, and the potential energy functions of the graphite system," The Journal of Chemical Physics, vol. 25, pp. 693-697, Oct 1956. 
[53] N. Levy, S. Burke, K. Meaker, M. Panlasigui, A. Zettl, F. Guinea, A. Neto, and M. Crommie, "Strain-induced pseudo-magnetic fields greater than 300 tesla in graphene nanobubbles," Science, vol. 329, no. 5991, p. 544, 2010.

[54] N. Mounet and N. Marzari, "First-principles determination of the structural, vibrational and thermodynamic properties of diamond, graphite, and derivatives," Physical Review B, vol. 71, p. 205214, May 2005.

[55] S. Amini, J. Garay, G. Liu, A. A. Balandin, and R. Abbaschian, "Growth of large-area graphene films from metal-carbon melts," Journal of Applied Physics, vol. 108, p. 094321, Nov 2010.

[56] R. K. Kirby, "Platinum - a thermal expansion reference material," International Journal of Thermophysics, vol. 12, pp. 679-685, Jul 1991.

[57] M. Neek-Amal and F. Peeters, "Buckled circular monolayer graphene: a graphene nano-bowl," Journal of Physics: Condensed Matter, vol. 23, p. $045002,2011$. 